\title{
Tactical Asset Allocation
}

\author{
Thomas J.Flavin \\ B.B.S., University of Limerick, 1993 \\ M.A., National University of Ireland, Maynooth, 1995
}

Submitted for the degree of Doctor of Philosophy

to University of York

Department of Economics and Related Studies

September 1998 


\begin{abstract}
In this thesis, we are concerned with developing asset allocation strategies that will allow investors to optimally choose their investment portfolio. In particular, we focus on tactical asset allocation strategies. This refers to the process whereby investors regularly revise the composition of their portfolios in response to changes in the wider economic environment. Such strategies have become increasingly popular in the aftermath of the 1987 stock market crash when invest ors who adopted this method of asset allocation were found to have fared much better than investors who held more conventional buy and hold portfolios.

We apply recently developed econometric techniques to help us build these asset allocation models. We work with Generalised Autoregressive Conditional Heteroscedasticity (GARCH) models which allow us to capture the time-varying risk inherent in financial markets. We develop a new parameterisation for the multivariate GARCH model which allows us to distinguish between long-run and short-run sources of financial market risk.

We begin the analysis in chapter 4 from the perspective of a UK investor wishing to hold only domestic assets. Using the portfolio theory of Markowitz(1952), we solve for the asset proportions in the optimal portfolio period by period.
\end{abstract}


In the following chapter, we extend the analysis to allow for international diversification. We address the problem from the viewpoint of both investors in the UK and US where each investor is allowed to hold domestic assets and the equity of the other country. We look at the importance of investor location and the degree of home country bias.

We subsequently extend the analysis in chapter 6 to allow the UK investor to hold an even greater number of non-domestic assets and investigate the importance of such assets in the portfolio and how they affect portfolio performance.

In chapters 7 and 8 , we allow macroeconomic factors to influence the asset allocation decision for both the domestic portfolio and the internationally diversified portfolio. The model developed in chapter 3 provides a neat way of conducting this analysis as we can jointly model the financial asset returns and macroeconomic factors. 


\section{Contents}

Declaration

1 Introduction

2 Portfolio Theory $\quad 29$

2.1 Mean-Variance Portfolio Analysis . . . . . . . . . . . 30

2.2 Two Fund Separation Theorem . . . . . . . . . . 37

2.3 Utility ..................... . . 40

3 ARCH and GARCH Models 42

3.1 Background ................... . . 42

3.2 Multivariate GARCH Models . . . . . . . . . . . . 46

3.2 .1 Vector $\mathrm{ARCH} \ldots \ldots \ldots \ldots$

3.2 .2 Diagonal ARCH . . . . . . . . . . . 48

3.2.3 Constant Conditional Correlations . . . . . . . 50 
3.2.4 Factor ARCH Models . . . . . . . . . . . . . 51

3.3 A New Representation . . . . . . . . . . . . . 53

4 Optimal Allocation of Domestic Assets for the UK Investor 56

4.1 Introduction . . . . . . . . . . . . . 56

4.2 Empirical Model . . . . . . . . . . . . 60

4.3 Data .......................... 61

4.4 Estimating the Model . . . . . . . . . . . . . . . 63

4.4.1 Convergence of the Log Likelihood Function . . . . 63

4.4 Results................... 64

4.4.3 Estimates of the Conditional Mean . . . . . . . 66

4.4.4 Estimates of the Conditional Covariance Matrix . . . 67

4.4 .5 Frontier Movements. . . . . . . . . . . 68

4.4.6 Optimal Portfolios ............. 71

4.5 Conclusion . . . . . . . . . . . . . . . 77

5 Optimal Asset Allocation with an International Perspective.

$\begin{array}{ll}\text { Is Investor Location Important? } & 91\end{array}$

5.1 Introduction . . . . . . . . . . . . . 91

5.2 Home Bias . . . . . . . . . . . . . . . . . . 94

5.3 Econometric Methodology and Data . . . . . . . . . 101

5.3 .1 The Model. . . . . . . . . . . . . 101 
$5.3 .2 \quad$ The Data . . . . . . . . . . . . . . . . . 102

5.4 Estimation Results . . . . . . . . . . . . . . . . . . 104

5.4 .1 UK Investor . . . . . . . . . . . . . . . . . . . 104

5.4 .2 US Investor . . . . . . . . . . . . . . . . . 108

5.5 Optimal Asset Allocation . . . . . . . . . . . . . . 111

5.5.1 Frontier Movements . . . . . . . . . . . . . . . . 111

5.5 .2 Optimal Portfolios . . . . . . . . . . . . . 112

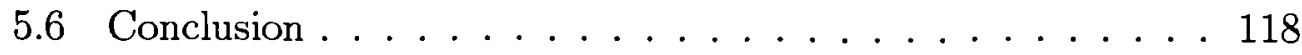

6 Global Asset Allocation 132

6.1 Introduction . . . . . . . . . . . . . . . . . 132

6.2 The Model and Data . . . . . . . . . . . . . 135

6.2.1 The Model . . . . . . . . . . . . . . . . . . . 135

6.2 .2 The Data . . . . . . . . . . . . . 136

6.3 Results and Analysis . . . . . . . . . . . . . . 138

6.3.1 Global Bond Portfolio . . . . . . . . . . . . . 138

6.3.2 European Equity Portfolio . . . . . . . . . . . . . . 145

6.3.3 Global Asset Allocation . . . . . . . . . . . . . 152

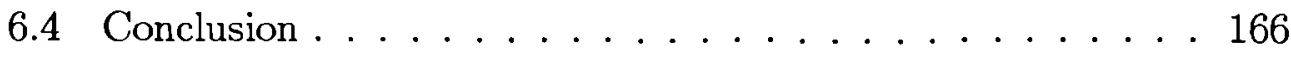

7 Macroeconomic Influences on the Optimal Allocation of Do-

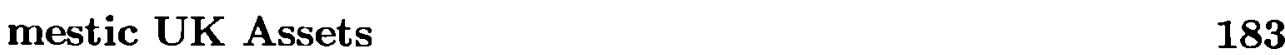


7.1 Introduction . . . . . . . . . . . . . . . . 183

7.2 Asset Returns and Macroeconomics . . . . . . . . . . 186

7.2.1 The Empirical Evidence . . . . . . . . . . . . 186

7.2 .2 Stock Returns and Inflation _ . . . . . . . . . 192

7.3 Empirical Model and Data . . . . . . . . . . . . . . 195

$7.3 .1 \quad$ Model . . . . . . . . . . . . . . . . . 195

$7.3 .2 \quad$ Data . . . . . . . . . . . . . . . . 197

7.4 Estimation Results . . . . . . . . . . . . . . . . . . 198

7.4 .1 Mean Matrices . . . . . . . . . . . . . . . . . 199

7.4 .2 Covariance Matrices . . . . . . . . . . . . . . . 199

7.4.3 Discussion of Results . . . . . . . . . . . . . . 200

7.4.4 Frontier Movements . . . . . . . . . . . . . . 203

7.4.5 Optimal Portfolios . . . . . . . . . . . 205

7.5 Conclusion . . . . . . . . . . . . . . . . 209

\section{Macroeconomic Influences on International Portfolio Selec-}

tion

8.1 Introduction . . . . . . . . . . . . . . . . 221

8.2 Macroeconomic Factors . . . . . . . . . . . . . . . . 223

8.3 The Model and Data . . . . . . . . . . . . . 226

8.3.1 The Model . . . . . . . . . . . . . . . . . . . 226

$8.3 .2 \quad$ Data . . . . . . . . . . . . . . . . . 228 
8.4 Estimation Results . . . . . . . . . . . . . . . . 228

8.4.1 Mean Matrices . . . . . . . . . . . 229

8.4 .2 Covariance Matrices . . . . . . . . . . . 230

8.4.3 Discussion of Results . . . . . . . . . . . . 233

8.5 Conclusion . . . . . . . . . . . . . . 236

9 Conclusion $\quad 238$

9.1 Review of Results . . . . . . . . . . . . . . . 238

9.2 Future Research . . . . . . . . . . . . . . . . . 246

$\begin{array}{ll}\text { Appendix } 1 & 249\end{array}$ 


\section{List of Tables}

4.1 Key features of optimal domestic portfolio . . . . . . . 72

4.2 Optimal buy and hold portfolios . . . . . . . . 72

4.3 Summary statistics for optimal unrestricted domestic portfolio 74

4.4 Summary statistics for optimal restricted domestic portfolio $\quad 75$

5.1 Summary statistics for optimal unrestricted UK portfolio . . 114

5.2 Summary statistics for UK restricted portfolio . . . . . . 115

5.3 Summary statistics for US unrestricted portfolio . . . . . . . 117

5.4 Summary statistic for optimal restricted US portfolio . . . 117

6.1 Summary statistics for the unrestricted global bond portfolio 144

6.2 Summary statistics for the restricted global bond portfolio . 144

6.3 Summary statistics for unrestricted European equity portfolio 150

6.4 Summary statistics for restricted European equity portfolio . 151

6.5 Summary statistics for unrestricted global asset portfolio . . 162

6.6 Summary statistics for restricted global asset portfolio . . . 164 
6.7 Global Asset Allocation for UK investor . . . . . . . . 165

7.1 Summary statistics for unrestricted portfolio with Macro influences . . . . . . . . . . . . . . . 206

7.2 Summary statistics for restricted portfolio with Macro influ-

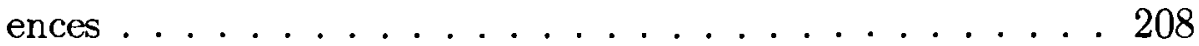

7.3 Macroenomic effects on mean asset holdings . . . . . . . . 209 


\section{List of Figures}

2-1 Identification of the optimal portfolio of risky assets . . . . . 38

4-1 Conditional variances of domestic assets . . . . . . . 81

4-2 Portfolio frontier movement at four yearly intervals . . . . . 82

4-3 Distribution of the time-varying portfolio frontiers . . . . . . 83

4-4 Portfolio frontier movement from July '81 - Dec. '81 . . . . . 84

4-5 Portfolio frontiers: conditional versus unconditional . . . . 85

4-6 Distribution of frontiers generated by differing conditional mean specifications ................ 86

4-7 Excess return and standard deviation of optimal domestic portfolio ................... 87

4-8 Unrestricted portfolio shares for each asset . . . . . . . 88

4-9 Restricted portfolio shares for each asset . . . . . . . . 89

4-10 Risk reduction performance of the time-varying portfolios . . 90

5-1 Conditional variances of UK financial assets . . . . . . . 121 
5-2 Conditional variances of US financial assets . . . . . . . . . 122

5-3 Mean time-varying portfolio frontiers for UK and US investors123

5-4 Distribution of portfolio frontiers for UK and US investors . 124

5-5 Excess return and standard deviation of the optimal UK port-

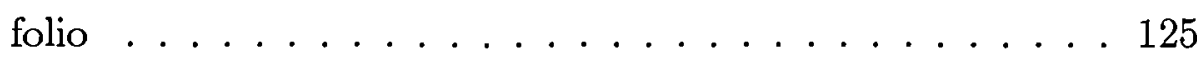

5-6 Excess return and standard deviation of the optimal US port-

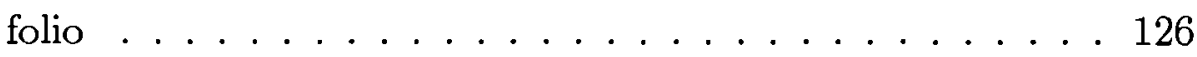

5-7 Sharpe Performance Indices for UK and US portfolios . . . . 127

5-8 Unrestricted portfolio shares for each asset in optimal UK portfolio ........................ 128

5-9 Restricted portfolio shares for each asset in optimal UK portfolio ........................... 129

5-10 Unrestricted portfolio shares for each asset in optimal US portfolio ...................... 130

5-11 Restricted portfolio shares for each asset in optimal US portfolio131

6-1 Conditional variances of government bonds . . . . . . . . 169

6-2 Unrestricted global bond portfolio . . . . . . . . . . . 170

6-3 Restricted global bond portfolio . . . . . . . . . . 171

6-4 Conditional variances of European equities . . . . . . . 172

6-5 Unrestricted portfolio of European equity . . . . . . . . 173

6-6 Restricted portfolio of European equity . . . . . . . . . . . 174 
6-7 Conditional variances of global financial assets . . . . . . . 175

6-8 Distribution of portfolio frontiers . . . . . . . . . 176

6-9 Comparison of mean portfolio frontiers with differing investment sets . . . . . . . . . . . . 177

6-10 Comparison of portfolio frontier distribution under differing investment sets ................... 178

6-11 Excess return and standard deviation of optimal portfolio of

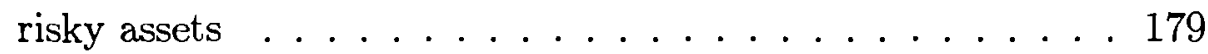

6-12 Sharpe Performance Index for optimal portfolio . . . . . . . 180

6-13 Unrestricted portfolio of global assets . . . . . . . . . . 181

6-14 Restricted portfolio of global assets . . . . . . . . . . 182

7-1 Conditional variances of UK financial assets with macro effects213

7-2 Distribution of portfolio frontiers . . . . . . . . 214

7-3 Comparison of distribution of portfolio frontiers with and without macro effects . . . . . . . . . . . 215

7-4 Unconditional versus conditional portfolio frontiers . . . . 216

7-5 Excess return and standard deviation on optimal portfolio . 217

7-6 Sharpe Performance Indices for optimal portfolios . . . . . . 218

7-7 Unrestricted portfolio of assets . . . . . . . . . . . . 219

7-8 Restricted portfolio of assets . . . . . . . . . . . 220 


\section{Acknowledgments}

There are a number of people who deserve special thanks for the help and support that they have offered during my study at University of York. First and foremost, thanks to my supervisor, Professor Mike Wickens, not alone for his invaluable and helpful insights from an economic viewpoint but also for providing me with the opportunity to work on a research project which provided vital financial support. I am also indebted to Dr. Peter N. Smith for his many helpful discussions and guidance.

Heartfelt thanks to all my teachers throughout my education from Knockanure N.S to Saint Michael's College, Listowel and onto University of Limerick and NUI, Maynooth, before finally arriving at University of York. Special thanks to Professor Geary and the staff of department of Economics, Maynooth and Jim Deegan at University of Limerick whose guidance and advice pointed me in this direction.

I must express my sincerest gratitude to all the friends that I have met during my years in York, unfortunately too many to mention by name. 
However, it would be remiss of me not to give special mention to Michele and Loredana, Etta, Manuel, Sigei and the inhabitants of the "Burnholme Orphanage" whose support in the early years was vital; 'mia vera amica' Anna whose friendship means so much; the Irish gang who made Tuesday nights something to look forward to; the Thursday night and Sunday morning footballers who provided a welcome respite from work; last but not least Teresa and Roberto who nursed me back to health. To all the others, your friendship is treasured just as much.

Finally, I can never adequately express my gratitude to my parents, brother and sisters for their concern and patience throughout my many years in various universities. Without your selfless and unquenchable support, it would have been impossible. Also the help and assistance of members of the extended family such as uncles, aunts, brothers-in-law, sister-in-law, nephews, niece and cousins is warmly appreciated. 


\section{Declaration}

The original work for this thesis was produced as part of a project that I worked on with my supervisor, Professor Mike Wickens, while I was employed as a research fellow by the University of York. Chapters 4 and 5 have been submitted for publication as joint work.

An earlier version of chapter 4 has been presented at the 1997 Money, Macro and Finance Annual meeting in University of Durham with the title Optimal Asset Allocation: A Multivariate GARCH Approach.

Chapter 5 has been presented at a PhD. student workshop at University of Southampton in May 1998 and also at the 1998 Money, Macro and Finance Annual meeting at Imperial College, London under the title $O p$ timal Asset Allocation with International Diversification. It has also been accepted for presentation at the forthcoming ASSET 98 meeting at University of Bologna.

A preliminary draft of chapter 7 entitled Optimal Asset Allocation and Macroeconomic Effects has been presented at the 1997 Irish Economic As- 
sociation Annual conference in Athlone.

All work, apart from the aforementioned joint chapters, is mine and any remaining errors are my sole responsibility. 


\section{Chapter 1}

\section{Introduction}

The main focus of this thesis is on the world of financial economics and the building of models incorporating recently developed econometric techniques. It is anticipated that our studies and results will appeal to both academics and financial market participants. We address questions that interest individual investors and large pension fund managers alike and demonstrate how developments in econometric tools can be married with advances in computing power to provide more realistic and insightful models which can be used to implement modern financial theory as it was originally intended.

The majority of the thesis is concerned with the problem of optimal asset allocation and the development of textbook theories of portfolio management to incorporate realistic market features. The best known of the traditional approaches are the mean-variance analysis of Markowitz (1952) 
and the capital asset pricing model (CAPM) of Sharpe(1964) and Lintner(1965). Markowitz assumes that investors wish to minimise the riskiness of the portfolio return subject to achieving a target rate of return while in the CAPM the optimal portfolio trades-off expected return and variance to maximise a - typically quadratic - welfare function in mean and variance. In implementing these models much of the early work assumed that risk, measured by the covariance matrix of asset excess returns, was constant or sufficiently slowly changing that it could be estimated by the unconditional matrix of past returns and then treated as though it would be constant for a fixed period in the future; see for example, Grubel(1968) and Levy \& Sarnat(1970). Now it is a widely accepted fact that the conditional second order moments violate this assumption and indeed vary from period to period. Our main innovation is that we allow the conditional covariance matrix to be time varying and estimate it in a comprehensive multivariate framework. This is a major advancement in that it recognises the importance of the second order moments and shows that in the presence of time-varying risk, the optimal portfolio needs to be re-balanced from period to period.

The original economic contributions made in this work fall into two categories. The first contribution of this thesis is in the development of econometric techniques. Many issues in finance and macroeconomics require modelling with a time-varying variance-covariance matrix and in a 
comprehensive multivariate framework. However, until Engle(1982) and Bollerslev(1986) introduced the family of (G)ARCH models, the econometric techniques capable of dealing with this problem were not available. Even then many interesting issues, such as asset allocation, which cry out for multivariate (G)ARCH models were still outside the realms of possibility due to the vast computational burden required to implement them empirically. However with increased computing power and more parsimonious parameterisations of the M-GARCH model, it is now possible to apply this econometric technique to many issues in financial markets. Our contribution is that we develop a new variant of a more parsimonious representation. By writing the second order moments in error correction format, we can distinguish between long-run and short-run sources of volatility. This also allows considerable flexibility in the conditional covariance matrix but remains economical in the number of parameters it uses.

The second contribution of this work appeals to financial economics. In chapters 4-8, we develop models that strive to optimally allocate an investor's funds among major classes of assets. The spirit of these asset allocation models is consistent with the pioneering work of Markowitz 1952.1959 who was the first to formulate and solve the mean-variance rule of port folio selection. Like Markowitz, we pay particular attention to mimimising the variance of the returns on our portfolio of assets subject to constraints such 
that the portfolio delivers a pre-specified target rate of return. These models are best described as tactical asset allocation models in that it is assumed that the investor has a short investment horizon. Tactical asset allocation strategies have been afforded an increased amount of attention in the aftermath of the October 1987 Stock Market crash, since investors who had adopted such strategies had smaller equity holdings in their portfolios and were relatively unharmed by the crash. Given the amazing advancements in computer technology during the 1990's, it is now feasible to construct myopic investment strategies based on risk management criteria.

In our studies we find compelling evidence for the uncorrelatedness of asset excess returns. On the other hand, we show that the second order moments of financial asset excess returns are much more forecastable and persistent than the level of the price movement. Taking both these findings into account, it makes more sense to construct portfolios based on risk minimisation subject to the portfolio delivering a target level of return over the risk-free rate, rather than attempting to maximise portfolio return.

The rest of the introduction comprises a brief preview of each subsequent chapter, outlining its main aims and original contributions. Each chapter contains a review of the pertinent associated literature and thus, this introduction avoids a major literature review.

Chapter 2 reviews the basic concepts of portfolio theory which are impor- 
tant for the original work undertaken later in the thesis. Section 2.1 presents the path breaking work of Markowitz(1952) in formulating a mean-variance rule for the selection of portfolios of assets. We deviate from Markowitz's original problem in two ways. Firstly we allow for unlimited short sales and secondly, we add a time dimension to the problem. Deriving this rule allows us to find a mathematical expression for the vector of asset weights in a given portfolio and for the variance (or standard deviation) of the portfolio returns solely in terms of asset expected returns, variances and covariances. Armed with these expressions, we are able to generate the portfolio frontier of risky assets. This is the locus of all points which for a given level of expected return minimises the variance of an asset or portfolio of assets. We then show how to identify and locate the optimal portfolio of risky assets. Section 2.2 recalls the two fund separation theorem of Tobin and shows how invoking the theorem in this context allows us to identify the two portfolios between which each investor, regardless of preferences, will allocate their total investable funds. Finally, section 2.3 discusses the conditions under which this mean-variance framework is consistent with expected utility maximisation. There are two occasions when this desirable result will hold and we look at the merits of each. We conclude the section by citing some empirical findings which support the stance that is adopted later in the original work. 
Chapter 3 looks at the econometric techniques employed in this thesis. We begin by looking at univariate $\mathrm{ARCH}$ and $\mathrm{GARCH}$ models and highlighting the major contribution that they have made to empirical work in financial economics. In the second sub-section, we look at their multivariate counterparts and stress the problems in implementing these models. The main problem arises from the vast number of potential parameters required for simultaneous estimation. We present the most general formulation of the model and then review some of the other more parsimonious representations that have been put forward in the literature to make the estimation of these models more feasible. Finally in section 3.3, we introduce a new parameterisation of the Multivariate GARCH model which is used extensively in the remainder of the thesis. The main innovation is that we write the conditional second order moments in error correction format, thereby enabling us to distinguish between long-run and short-run sources of asset volatility.

Chapter 4 is the first original chapter of the thesis. Using the concepts of portfolio theory outlined in chapter 2 and the M-GARCH model of chapter 3 , we build a tactical asset allocation model from the perspective of a $U K$ investor who is interested in holding a portfolio of domestic assets. We estimate our model and generate the portfolio frontiers facing this investor in each period of the analysis. Period by period, we locate the optimal portfolio 
of risky assets and identify the proportions in which each asset should be held in order for the investor to hold this portfolio. By identifying the composition of the risky portfolio and assuming that a riskless asset exists, we have identified two portfolios. By invoking the two fund separation theorem, we believe that each investor irrespective of preferences will hold a combination of these. This allocation of funds among the risky assets is calculated under two different investment strategies. Firstly, the allocation is unrestricted so that the investor is free to hold unlimited short positions in any of the assets. This often leads to an excessively volatile allocation. Our second strategy overcomes this problem by restricting each asset holding to be non-negative. This is consistent with the real world as UK mutual fund managers have this constraint imposed upon them by legislation.

Chapter 5 extends the analysis of the previous study to include the possibility of investing in a foreign asset. This allows us to address issues of benefits accruing from international diversification and highlight the home asset bias puzzle. This study begins with a review of the home bias literature which stresses the stylised fact that domestic investors tend to hold the majority of their portfolios in domestic assets despite many studies showing that there are unexploited gains to be reaped from diversifying across international markets. This study again utilises the M-GARCH model of chapter 3 and is conducted from the perspective of both UK and US in- 
vestors. We find that US investors enjoy a risk-return advantage over their UK counterparts in that the same expected return is associated with lower risk in the US than in the UK given the investment opportunity set. There is also significant evidence of contagion effects between markets, both domestically and internationally. Having identified the optimal portfolio of risky assets for each period of the analysis, we conclude that the home bias problem exists for both investors but is more acute in the US.

In chapter 6 , we present a global asset allocation strategy from the perspective of a UK investor. One of the potential shortcomings of using MGARCH models to build tactical asset allocation models is that we have to limit the number of assets in the investment opportunity set due to the sheer dimensionality of the model. We overcome this problem by adopting a multi-stage allocation process. At the initial stage, we form an optimal global bond portfolio and an optimal portfolio of European equity for each period of the analysis. Using the optimal proportions suggested by our model, each of these portfolios enter the final allocation decision as a single asset. This allows us to extend the investment opportunity set to ten assets. We find that extending the number of assets offers superior risk-return combinations to the UK investor as opposed to the study of the previous chapter with the optimal portfolios having much better performance levels. Furthermore, there is strong evidence of volatility spillovers between mar- 
kets, though these are most pronounced between international stockmarkets. We find that stockmarkets and government bond markets are largely segmented from each other in that there is little evidence that volatility is transmitted from equity to bond markets. This is a reason for holding both asset classes in the portfolio. The holding of assets in the optimal portfolios suggest that there are large benefits available to the UK investor who is willing to hold non-UK assets. On average, the optimal portfolio contains eight risky assets, six of which are foreign assets. Consequently, the home bias problem is larger that previously thought.

We demonstrate another advantage of our proposed M-GARCH model in chapter 7 by allowing macroeconomic variables to exert an influence on the asset allocation strategy through their covariances with the financial assets under consideration. This is achieved by jointly modelling the asset returns and the macroeconomic factors. We demonstrate that the way in which we define the parameter matrices in the second order moments of the model has an important bearing on the time lag with which the macro factors influence the allocation process. We illustrate this joint modelling technique from the perspective of a UK investor who again is only interested in holding domestic assets (as in chapter 4). The chapter begins with a review of the literature pertaining to the ability (and use) of macroeconomic factors to predict future financial asset price movements. At the end of this 
section, we pay particular attention to the puzzling empirical findings on the relationship between stock returns and inflation. This is important as our illustration uses domestic inflation as the macro variable. Our results suggest that taking the inflation effect into account yields more attractive risk-return combinations, especially for an investor who may be interested in holding the minimum variance portfolio. Furthermore, the proportions in which individual assets are held in the optimal portfolio change following the inclusion of macroeconomic factors in the analysis.

Chapter 8 extends the analysis of chapter 5 by allowing macroeconomic factors to influence the portfolio selection decision of the UK investor who has the opportunity to invest in US equity as well as home assets. We use a standard mean-variance framework to provide a rationale for the macroeconomic variables included in the analysis. These variables are domestic and foreign inflation and the foreign exchange rate. In contrast to the portfolio of domestic assets, we find that inflation variables play no role in determining the variances and covariances of the financial asset excess returns in this context. This is interpreted as evidence that holding foreign assets in the portfolio provides the UK investor with an adequate hedge against inflation. Consequently, when building an internationally diversified tactical asset allocation strategy, we should not be concerned about inflation effects. It is sufficient to model only the financial asset excess returns as in chapter 5 . 
Finally, chapter 9 concludes. We briefly attempt to summarise our main findings and set out future avenues of research that have been signposted by the material produced for this thesis. 


\section{Chapter 2}

\section{Portfolio Theory}

This section aims to give a brief outline of modern portfolio theory. It is not intended to be a comprehensive treatment of the subject but rather a presentation of the concepts and equations that are important for the work which follows. ${ }^{1}$

Markowitz (1952) is generally credited with being the first proponent of modern portfolio theory. In formulating and solving the risk versus return rule, Markowitz had first to disprove the theories that were in existence and in common use at the time. The widely accepted principle of the day was that an investor should choose a portfolio of assets by maximising the discounted expected returns. This is not to suggest that these economists totally ignored the concept of risk. Typically, risk was accounted for, e.g.

\footnotetext{
${ }^{1}$ For a detailed treatment of Portfolio Theory, the reader is referred to Huang and Litzenberger (1988) or Ingersoll (1987).
} 
Keynes(1936) or Hicks(1939), by including a risk premium in the expected future asset returns. Markowitz argues that if this were the correct way to allocate assets, then the optimal portfolio should only contain one asset, i.e. the asset with the highest discounted flow of expected future returns. Of course, this is inconsistent with the observed phenomenon of diversified portfolios. Therefore, such a simple rule has to be rejected.

Markowitz(1952) develops a rule that is entirely consistent with diversification. This rule is known as the "mean-variance" rule. It shows that in trying to reduce the variance of a portfolio, one must not alone diversify but importantly diversify over securites with low return covariance. Later in 1959, Markowitz shows that this type of portfolio selection is firmly grounded as rational choice under uncertainty.

The standard textbook treatment of portfolio selection theory is presented in a static framework. However, for reasons that will become obvious in the remainder of this thesis, we add a time dimension to the problem and its solution.

\subsection{Mean-Variance Portfolio Analysis}

Primarily as a way of introducing notation, we state how we obtain the asset allocation. Since forecasts of equity and bond returns are highly inaccurate due to their near serial independence compared with forecasts of 
their covariance structure which are highly serially correlated, we focus on choosing a minimum variance portfolio of excess returns over the risk-free rate. In effect this give ${ }^{5}$ us the tangency portfolio of risky assets.

Thus the optimal portfolio is obtained using mean-variance analysis as follows ${ }^{2}$. We assume that investors are forming their portfolios for one period only, period $t$, using information up to and including period $t-1$. Let $\mathbf{w}_{t}$ be an $n x 1$ vector whose components $w_{1 t} \ldots . . w_{n t}$ denote the proportion of an individual's wealth allocated to the $i$ th asset in the portfolio to be held in period $t$, where $i=1,2, \ldots . n$. Since it is assumed that all funds are invested, the sum of the weights must equal one, $\sum w_{i t}=1$. $\mathbf{i}$ is an $n x 1$ vector of ones and superscript / denotes the transpose of a vector or a matrix. $\mathbf{r}_{t}$ denotes an $n x 1$ vector of returns $r_{1 t} \ldots . r_{n t}$ where it is assumed that not all elements of $\mathbf{r}_{t}$ are equal. It is assumed that the conditional mean of $\mathbf{r}_{t}$ is $\boldsymbol{\mu}_{t \quad 1}$ and the conditional variance is $\boldsymbol{\Omega}_{t-1}$ is the $n x n$ covariance matrix with entries $\sigma_{i j, t}\{i, j=1,2, \ldots n\}$. It is assumed that $\boldsymbol{\Omega}_{t}$ is non-singular, i.e. no individual asset return is perfectly correlated with the return on a portfolio made up of the remaining assets and that none of the assets or portfolios of assets are riskless. Of course, we know that the covariance matrix must be positive definite since the variances of risky portfolios are strictly positive.

In formulating mean-variance portfolios, the key assumption is that an

\footnotetext{
${ }^{2}$ This formulation follows Constantinides and Malliaris (1995) which in turn relies heavily on Roll (1977). It is does not claim to be original.
} 
investor's preferences can be represented by a utility function defined over the mean and variance of the return to a portfolio, $V\left(R_{p}, \sigma_{p}^{2}\right)$. Using standard notation, we know that the expected return and variance of a portfolio are given by:

$$
\begin{gathered}
R_{p t}=\mathbf{w}_{t}^{\prime} \mathbf{r}_{t}=\sum w_{i t} r_{i t} \\
\sigma_{p t}^{2}=\mathbf{w}_{t}^{\prime} \Omega_{t} \mathbf{w}_{t}=\sum \sum w_{i t} w_{j t} \sigma_{i j, t} .
\end{gathered}
$$

The standard assumption is that investors prefer higher returns and smaller variances. Therefore, we are interested in finding the asset allocation that produces the portfolio with the minimum level of risk for a given level of expected return. This set of portfolios is termed mean-variance efficient. Following in the spirit of Markowitz'(1952) formulation, we first find the minimum variance portfolios in the absence of a riskless asset. This problem can be stated as:

Minimise $\quad \mathbf{w}_{t}^{\prime} \boldsymbol{\Omega}_{t} \mathbf{w}_{t}$ subject to:

$$
\begin{aligned}
& \mathbf{w}_{t}^{\prime} E\left(\mathbf{r}_{t}\right)=R_{p t} \\
& \mathbf{w}_{t}^{\prime} \mathbf{i}=1 .
\end{aligned}
$$

Unlike Markowitz(1952) we have not imposed any constraint on the sign 
of the asset weights so short sales are allowed in the analysis. It is also worth noting that the investor's preferences do not enter into the problem explicitly and unlike Tobin(1959) there is no cash (or other riskless asset) considered here. To mathematically derive the solution to this portfolio selection problem, we begin by forming the Lagrangian function

$$
L_{t}=\mathbf{w}_{t}^{\prime} \Omega_{t} \mathbf{w}_{t}-\lambda_{1 t}\left(\mathbf{w}_{t}^{\prime} \mathbf{r}_{t}-R_{p t}\right)-\lambda_{2 t}\left(\mathbf{w}_{t}^{\prime} \mathbf{i}-1\right)
$$

The first order conditions are

$$
\begin{gathered}
\frac{\delta L_{t}}{\delta w_{t}}=2 \Omega_{t} \mathbf{w}_{t}-\lambda_{1 t} \mathbf{r}_{t}-\lambda_{2 t} \mathbf{i}=0 \\
\frac{\delta L_{t}}{\delta \lambda_{1 t}}=R_{p t}-\mathbf{w}_{t}^{\prime} \mathbf{r}_{t}=0 \\
\frac{\delta L_{t}}{\delta \lambda_{2 t}}=1-\mathbf{w}_{t}^{\prime} \mathbf{i}=0
\end{gathered}
$$

From equation (2.5) we get

$$
\mathbf{w}_{t}=\frac{1}{2} \Omega_{t}^{1}\left(\lambda_{1 t} \mathbf{r}_{t}+\lambda_{2 t} \mathbf{i}\right)=\frac{1}{2} \Omega_{t}^{-1}\left[\begin{array}{ll}
\mathbf{r}_{t} & \mathbf{i}
\end{array}\right]\left[\begin{array}{l}
\lambda_{1 t} \\
\lambda_{2 t}
\end{array}\right]
$$

From equations (2.6) and (2.7), 


$$
\left[\begin{array}{ll}
\mathbf{r}_{t} & \mathbf{i}
\end{array}\right]^{\prime} \mathbf{w}_{t}=\left[\begin{array}{l}
R_{p t} \\
1
\end{array}\right]
$$

Premultiply both sides of equation (2.8) to get,

$$
\left[\begin{array}{ll}
\mathbf{r}_{t} & \mathbf{i}
\end{array}\right]^{\prime} \mathbf{w}_{t}=\frac{1}{2}\left[\begin{array}{ll}
\mathbf{r}_{t} & \mathbf{i}
\end{array}\right]^{\prime} \boldsymbol{\Omega}_{t}^{-1}\left[\begin{array}{ll}
\mathbf{r}_{t} & \mathbf{i}
\end{array}\right]\left[\begin{array}{l}
\lambda_{1 t} \\
\lambda_{2 t}
\end{array}\right]=\left[\begin{array}{l}
R_{p t} \\
1
\end{array}\right]
$$

For convenience denote

$$
\boldsymbol{\Gamma}_{t}=\left[\begin{array}{ll}
\mathbf{r}_{t} & \mathbf{i}
\end{array}\right]^{\prime} \boldsymbol{\Omega}_{t}^{1}\left[\begin{array}{ll}
\mathbf{r}_{t} & \mathbf{i}
\end{array}\right]
$$

the $2 x 2$ symmetric matrix with entries

$$
\left[\begin{array}{ll}
a_{t} & b_{t} \\
b_{t} & c_{t}
\end{array}\right]=\left[\begin{array}{cc}
\mathbf{r}_{t}^{\prime} \boldsymbol{\Omega}_{t}^{-1} \mathbf{r}_{t} & \mathbf{r}_{t}^{\prime} \boldsymbol{\Omega}_{t}^{1} \mathbf{i} \\
\mathbf{r}_{t}^{\prime} \boldsymbol{\Omega}_{t}{ }^{1} \mathbf{i} & \mathbf{i}^{\prime} \boldsymbol{\Omega}_{t}^{-1} \mathbf{i}
\end{array}\right]
$$

The next step is to establish that $\boldsymbol{\Gamma}$ is positive definite. For any $y_{1}, y_{2}$ such that at least one of the elements $y_{1}, y_{2}$ is non-zero, it is clear that

$$
\left[\begin{array}{ll}
\mathbf{r}_{t} & \mathbf{i}
\end{array}\right]\left[\begin{array}{l}
y_{1} \\
y_{2}
\end{array}\right]=\left[y_{1} \mathbf{r}_{t}+y_{2} \mathbf{i}\right]
$$

is a non-zero n-vector because, by assumption, the elements of $\mathbf{r}_{t}$ are not 
all equal.

Then $\boldsymbol{\Gamma}$ is positive definite because

$$
\begin{aligned}
{\left[\begin{array}{ll}
y_{1} & y_{2}
\end{array}\right] \boldsymbol{\Gamma}_{t}\left[\begin{array}{l}
y_{1} \\
y_{2}
\end{array}\right] } & =\left[\begin{array}{ll}
y_{1} & y_{2}
\end{array}\right]\left[\begin{array}{ll}
\mathbf{r}_{t} & \mathbf{i}
\end{array}\right]^{\prime} \boldsymbol{\Omega}_{t}^{-1}\left[\begin{array}{ll}
\mathbf{r}_{t} & \mathbf{i}
\end{array}\right]\left[\begin{array}{l}
y_{1} \\
y_{2}
\end{array}\right] \\
& \left.=\left[y_{1} \mathbf{r}_{t}+y_{2} \mathbf{i}\right]^{\prime} \mathbf{\Omega}_{t}^{1}\left[y_{1} \mathbf{r}_{t}+y_{2} \mathbf{i}\right]\right\rangle 0
\end{aligned}
$$

by the positive definiteness of $\boldsymbol{\Omega}_{t}{ }^{1}$.

Substituting $\boldsymbol{\Gamma}$ into equation (2.10) yields

$$
\frac{1}{2} \boldsymbol{\Gamma}_{t}\left[\begin{array}{l}
\lambda_{1 t} \\
\lambda_{2 t}
\end{array}\right]=\left[\begin{array}{l}
R_{p t} \\
1
\end{array}\right]
$$

This allows us to solve for the multipliers since $\Gamma$ is non-singular and its inverse exists. Therefore

$$
\frac{1}{2}\left[\begin{array}{c}
\lambda_{1 t} \\
\lambda_{2 t}
\end{array}\right]=\Gamma_{t}^{1}\left[\begin{array}{l}
R_{p t} \\
1
\end{array}\right] .
$$

Combining these results, we are now in a position to solve for the nvector of portfolio weights that minimise the variance of a portfolio with a given mean return. 


$$
\begin{aligned}
\mathbf{w}_{t} & =\frac{1}{2} \boldsymbol{\Omega}_{t}^{1}\left[\begin{array}{ll}
\mathbf{r}_{t} & \mathbf{i}
\end{array}\right]\left[\begin{array}{l}
\lambda_{1 t} \\
\lambda_{2 t}
\end{array}\right] \\
& =\boldsymbol{\Omega}_{t}^{1}\left[\begin{array}{ll}
\mathbf{r}_{t} & \mathbf{i}
\end{array}\right] \boldsymbol{\Gamma}_{t}^{1}\left[\begin{array}{l}
R_{p t} \\
1
\end{array}\right] .
\end{aligned}
$$

We can now compute the variance of any minimum variance portfolio with a given mean return, $R_{p}$.

$$
\begin{aligned}
\sigma_{p t}^{2} & =\mathbf{w}_{t}^{\prime} \boldsymbol{\Omega}_{t} \mathbf{w}_{t} \\
& =\left[\begin{array}{ll}
R_{p t} & 1
\end{array}\right] \boldsymbol{\Gamma}_{t}^{1}\left[\begin{array}{ll}
\mathbf{r}_{t} & \mathbf{i}
\end{array}\right]^{\prime} \boldsymbol{\Omega}_{t}^{1} \boldsymbol{\Omega}_{t} \boldsymbol{\Omega}_{t}^{1}\left[\begin{array}{ll}
\mathbf{r}_{t} & \mathbf{i}
\end{array}\right] \boldsymbol{\Gamma}_{t}^{1}\left[\begin{array}{l}
R_{p t} \\
1
\end{array}\right] \\
& =\left[\begin{array}{ll}
R_{p t} & 1
\end{array}\right] \boldsymbol{\Gamma}_{t}^{1}\left[\begin{array}{c}
R_{p t} \\
1
\end{array}\right] \\
& =\left[\begin{array}{ll}
R_{p t} & 1
\end{array}\right] \frac{1}{\Delta}\left[\begin{array}{cc}
c & -b \\
-b & a
\end{array}\right]\left[\begin{array}{l}
R_{p t} \\
1
\end{array}\right] \\
& =\frac{1}{\Delta_{t}}\left(a_{t}-2 b_{t} R_{p t}+c_{t} R_{p t}^{2}\right),
\end{aligned}
$$

where $\Delta=a_{t} c_{t}-b_{t}^{2}$. It should be noted that $\Delta_{t}$ is strictly positive by the Cauchy-Schwarz inequality since we have assumed that $\boldsymbol{\Omega}_{t}$ is non-singular and all assets do not have the same mean.

The relationship between the variance of the minimum variance portfolio 
and the expected mean return given by equation (2.18) is expressed as a parabola and is called the minimum variance portfolio frontier. In meanstandard deviation space, the relation is expressed as a hyperbola.

Having drawn the portfolio frontier in mean-standard deviation space, we can find the optimal risky portfolio as the point of tangency between the frontier and a line drawn from the risk free rate, called the Capital Market Line, see figure 2-1. We can then proceed to invoke the Two Fund Separation Theorem to infer that an investor will only invest in a combination of these two mutual funds i.e. the optimal risky portfolio and the risk free asset.

\subsection{Two Fund Separation Theorem}

This section is devoted to the two-fund separation theorem. This is a very important result for asset allocation models. While the mathematics are relatively simple, its economic implication and applications are far reaching. Its most significant contribution is that it establishes that the entire minimum variance portfolio frontier can be generated by any two distinct frontier portfolios.

Let $P_{a}$ and $P_{b}$ be two minimum variance portfolios with mean returns of $R_{a}$ and $R_{b}$ respectively, such that $R_{a} \neq R_{b}$.

(1) Then every minimum variance portfolio, $P_{c}$, is a linear combination of $P_{a}$ and $P_{b}$; 
Portfolio Frontier

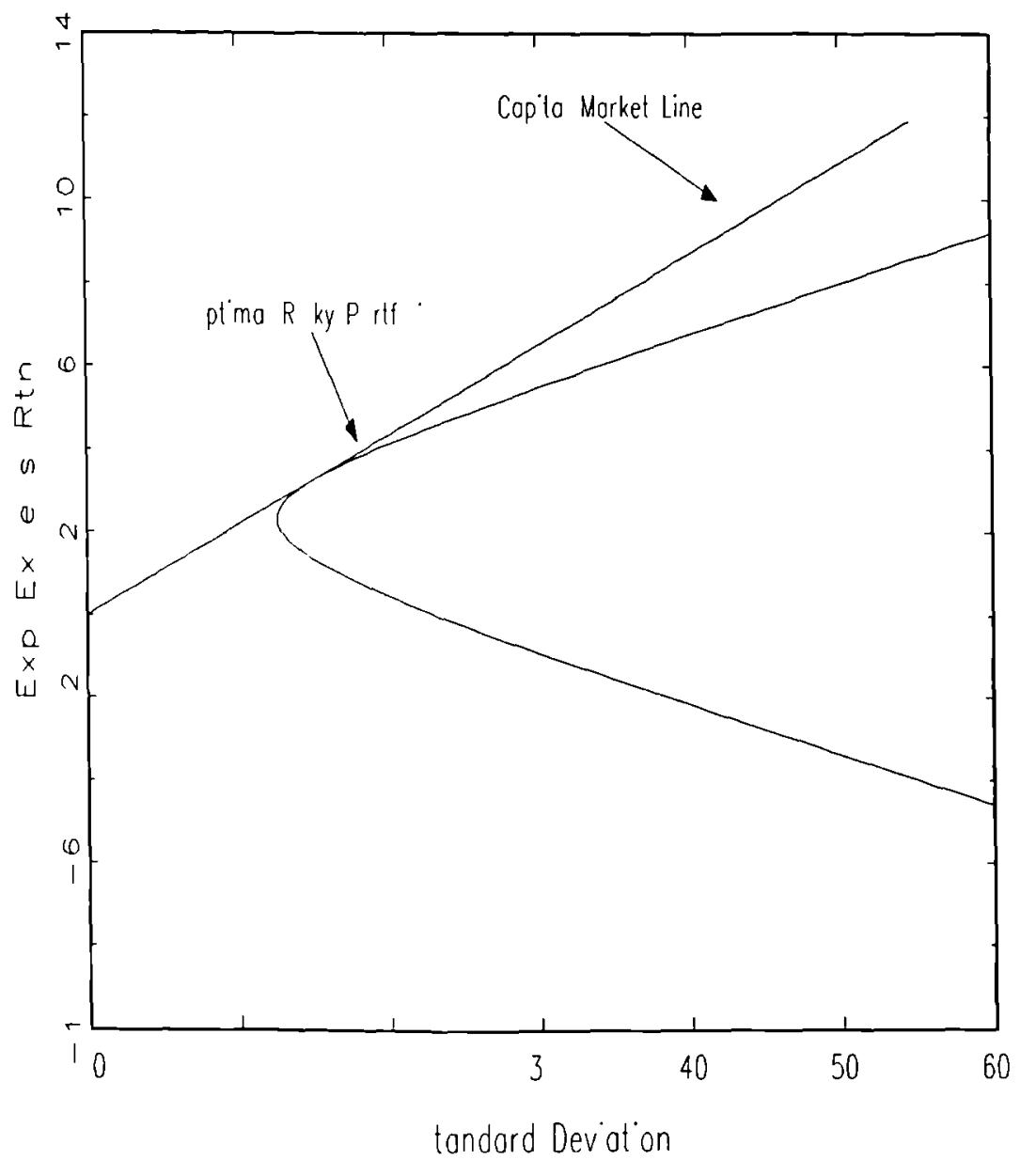

Figure 2-1: Identification of the optimal portfolio of risky assets 
(2) Conversely, every portfolio which is a linear combination of $P_{a}$ and $P_{b}$ is a minimum variance portfolio;

(3) In particular, if $P_{a}$ and $P_{b}$ are minimum variance efficient frontiers, then $\alpha P_{a}+(1-\alpha) P_{b}$ is a minimum variance efficient portfolio for $0 \leq \alpha \leq 1 .^{3}$

This theorem was discovered by Tobin (1958) who demonstrated the usefulness of the result using only two assets - cash and a risky asset - but argued that no major alterations are necessary even if there are many risky assets since these can be viewed as a single composite asset.

This provides a strong foundation for this work where risky assets will be viewed as a single mutual fund and the representative investor will choose to allocate funds between the riskless asset and the optimal portfolio of all risky assets. This neat result shows that an investor choosing a meanvariance efficient portfolio needs only to hold a linear combination of any two portfolios that are themselves mean-variance efficient. In this way, the investor has only to hold two assets to achieve the optimal combination of risk and return and not the $n$ original assets.

\footnotetext{
${ }^{3}$ Mathematical proofs of each of these three points are provided in Constantinides and Malliaris (1995)
} 


\subsection{Utility}

Up to now we have said very little about the consistency of the meanvariance analysis and expected utility maximisation. There are two conditions when this desirable result will hold. Firstly, for arbitrary distributions, we can assume quadratic utility. If an investor has a quadratic utility function defined solely over end-of-period wealth, then expected utility depends solely on the mean and variance of return. Furthermore, it can be shown that for risk averse utility functions and outcomes confined to the increasing utility range, then only the mean-variance efficient portfolios can be optimal.

Assuming quadratic utility has two drawbacks, namely the properties of satiation and increasing absolute risk aversion. The satiation property means that after a certain point, increased wealth leads to reduced utility which is not consistent with either theory or observed behaviour. Likewise the property of increasing absolute risk aversion is also inconsistent with economic theory in that it implies that risky assets are inferior goods.

The alternative approach is to assume that asset returns are multivariate normally distributed. Fama has cautiously accepted this as a working hypothesis for asset returns measured at monthly horizons. When this condition holds, then for any arbitrary set of preferences, the mean-variance analysis will be consistent with expected utility maximisation. This is the 
more common approach taken in the literature and is the approach used in the empirical investigation which follows ${ }^{4}$. While this is a more common justification for adopting the mean-variance framework, there is little evidence to suggest that this assumption holds true (Pulley(1981)).

The adoption of the mean-variance framework has been defended by Levy and Markowitz(1979), Pulley(1981), Kroll, Levy and Markowitz(1984) among others. Levy and Markowitz(1979) showed that the mean-variance rule yielded almost identical results to those found by maximising expected utility for various utility functions and historical distributions of returns. Later work by Pulley(1981) and Kroll, Levy and Markowitz(1984) confirm these findings, i.e. the optimal mean-variance portfolio was most often the portfolio which maximised expected utility regardless of the utility function employed. Taking these results into account, we can confidently proceed to utilise the mean-variance analysis to undertake our asset allocation studies.

\footnotetext{
${ }^{4}$ Ingersoll (1987) provides a concise, yet useful treatment of this topic.
} 


\section{Chapter 3}

\section{ARCH and GARCH Models}

\subsection{Background}

Until the beginning of the early 1980's, most empirical models concentrated on the conditional first moments of the model with any time variation in higher order moments conveniently ignored. However, as economic theory attributed an increasingly important role for uncertainty and risk premia in the world of macroeconomics and finance, it became necessary for empiricists to develop new econometric time series techniques capable of dealing with time variation in the second order moments of models. One such model was developed by Engle(1982). This is universally known as the Autoregressive Conditional Heteroskedasticity (ARCH) model. The major advancement of this model is in its ability to distinguish between conditional 
and unconditional second order moments e.g. while in many economic applications the unconditional variance-covariance matrix may be time invariant, the conditional variances and covariances quite often exhibit significant relationships with past states of nature. The ability of the model to capture this temporal dependence is a major step forward in helping us to understand many vitally important issues in financial economics.

Taking a simple $\operatorname{ARCH}(q)$ model as an example - where $q$ determines the length of time for which the shock persists in conditioning the variance of subsequent errors - we write it as follows:

$$
\begin{aligned}
y_{t} & =\mathbf{x}_{t} \boldsymbol{\beta}^{\prime}+\epsilon_{t}, t=1, \ldots, T \\
\epsilon_{t} & \mid \Psi_{t 1} \sim N\left(0, h_{t}\right) \\
h_{t} & =\alpha_{0}+\alpha_{1} \epsilon_{t}^{2}+\ldots+\alpha_{q} \epsilon_{t-q}^{2} \\
\alpha_{0} & >0, \alpha_{i} \geq 0 \quad i=1, . ., q .
\end{aligned}
$$

The first order moment equation is a standard regression model where $\mathbf{x}_{t}$ is an $m x 1$ vector of exogenous variables, $\boldsymbol{\beta}$ is an $m x 1$ vector of regression parameters while $\boldsymbol{\epsilon}_{t}$ is the error term. The key feature of this model is that the errors are distributed with zero mean but have a non-constant variance. The time-varying variance is a function of the information set available at 
time t-1, $\Psi_{t}$, and has a particular functional form that relates the current value of the variance to past squared errors. This functional form allows for the phenomenon of volatility clustering i.e. the observation that large errors are followed by large errors while small errors are followed by small errors. This behaviour is commonly observed in financial data especially in stock markets where we observe periods of sustained turbulence and other periods of relative tranquility. The restrictions on the signs of the $\alpha^{\prime} s$ ensure that the conditional variances are strictly positive.

However, in many applications of this model, it was discovered that a large value of $q$ was required to model the conditional second order moments. This required the estimation of a large number of parameters subject to lots of inequality restrictions. In an attempt to overcome this perceived shortcoming of the model, Bollerslev(1986) proposed an alternative but closely related model called the Generalized Autoregressive Conditional Heteroskedasticity GARCH model. This has proven itself to be a very useful extension to the original $\mathrm{ARCH}$ model in empirical work. A $\operatorname{GARCH}(p, q$ model can be specified as follows with $p$ determining the autoregressive order of the model and $q$ determining the number of lagged dependent variables to include when modelling the conditional variance. 


$$
\begin{aligned}
& y_{t}=\mathbf{x}_{t} \boldsymbol{\beta}^{\prime}+\epsilon_{t}, t=1, \ldots, T \\
& \epsilon_{t} \quad \mid \Psi_{t 1} \sim N\left(0, h_{t}\right) \\
& h_{t}=\alpha_{0}+\gamma_{1} h_{t}+\ldots+\gamma_{p} h_{t p}+\alpha_{1} \epsilon_{t 1}^{2}+\ldots+\alpha_{q} \epsilon_{t-q}^{2} \\
& \alpha_{0}>0 ; \gamma_{j} \geq 0 j=1, \ldots p ; \alpha_{2} \geq 0 i=1, . ., q .
\end{aligned}
$$

Again, the conditional mean equation can be interpreted as before. In this model, however, the conditional variance is not only a function of past squared errors but also of past values of the conditional variance itself. Once more, the restrictions on the $\alpha$ and $\gamma$ parameters ensure that the variance is strictly positive.

The main benefit of the GARCH model is the fact that in empirical applications quite low orders of $p$ and $q$ have been found to be sufficient in modelling most financial time series e.g. French, Schwert and Stambaugh(1987, Day and Lewis 1992) and Engle and Mustafa(1992). In fact, Bera and Higgins 1993 observe that;

"In applied work, it has been frequently demonstrated that the GARCH 1,1 process is able to represent the majority of financial time series. A data set which requires a model of order greater than GARCH 1,2 or GARCH 2,1 is very rare" 


\subsection{Multivariate GARCH Models}

Of course, both of these models are univariate in nature. However, there are several issues in financial economics that require modelling in a multivariate structure. The motivation for multivariate (G)ARCH stems from the fact that many economic variables react to the same information set and hence tend to have non-zero covariances conditional on the information set. Therefore, there are gains in efficiency to be exploited by modelling such issues as a multivariate system. Bollerslev, Engle and Nelson(1994) observe that;

"Financial market volatility moves together over time across assets and markets. Recognising this commonality through a multivariate framework leads to obvious gains in efficiency. Several interesting issues....also call for an explicit multivariate $\mathrm{ARCH}$ approach in order to capture the temporal dependencies in the conditional variances and covariances" (pp 3002).

\subsubsection{Vector ARCH}

However, the computational burden involved in estimating multivariate (G)ARCH models is quite considerable and is often sufficiently cumbersome to prevent its empirical application. This is due to the vast number of potential parameters requiring simultaneous estimation in the most 
general formulation of the $\operatorname{GARCH}(p, q)$ structure. Bearing in mind the earlier quotation taken from Bera and Higgins(1993), we begin by looking at $\operatorname{GARCH}(1,1)$ models. The most general form of this model can be represented by;

$$
\begin{aligned}
\mathbf{r}_{t} & =\boldsymbol{\alpha}+\boldsymbol{\xi}_{t} \\
\boldsymbol{\xi}_{t} & \mid \Psi_{t 1} \sim N\left(0, \mathbf{H}_{t}\right) \\
\operatorname{vech}\left(\mathbf{H}_{t}\right) & =\mathbf{W}+\operatorname{\Sigma S} \operatorname{vech}\left(\mathbf{H}_{t-1}\right)+\operatorname{\Sigma T} \operatorname{vech}\left(\boldsymbol{\xi}_{t-1} \boldsymbol{\xi}_{t-1}^{\prime}\right)
\end{aligned}
$$

where vech(.) is the vector half operator which stacks the lower triangle of a square matrix into a column vector. $\mathbf{H}_{t}$ is the time-varying conditional covariance matrix of asset excess returns. Since $\mathbf{H}_{t}$ is symmetric, vech $\left\{\mathbf{H}_{t}\right\}$ contains all the unique elements of the matrix. $\mathbf{r}_{t}$ is an $n x 1$ vector of asset excess returns over the risk free rate; $\boldsymbol{\alpha}$ is a vector of ones; $\boldsymbol{\xi}_{t}$ is a $n x 1$ vector of error terms. Concentrating on the second moment equation of 3.3 , we begin to get a feel for the dimensionality of the problem. $\mathbf{W}$ is a $[n(n+1) / 2 x 1]$ vector while both $\mathbf{S}$ and $\mathbf{T}$ are $[n(n+1) / 2 x n(n+1) / 2\}$ matrices and $n$ is the number of financial assets in the problem. It is clear that the potential number of parameters for estimation in this formulation is overwhelming. Even when there are only three assets, this specification 
of the conditional second moments requires the simultaneous estimation of 78 parameters, while for $n=5$ the number of parameters in this formulation grows to 465 .

This is clearly not a feasible working model and consequently empiricists have been forced to seek out alternative parameterisations of the conditional variance-covariance matrix in order to restrict the number of parameters required for estimation.

\subsubsection{Diagonal ARCH}

The first of these restricted models was proposed by Bollerslev, Engle and Wooldridge(1988 and is often referred to as Diagonal ARCH. This name has been adopted as because the $\mathbf{S}$ and $\mathbf{T}$ matrices in equation (3.3) are taken to be diagonal. It is easiest to write this model in terms of Hadamard products.

$$
\begin{aligned}
\mathbf{r}_{t} & =\boldsymbol{\alpha}+\boldsymbol{\xi}_{t} \\
\boldsymbol{\xi}_{t} & \mid \Psi_{t 1} \sim N\left(0, \mathbf{H}_{t}\right) \\
\mathbf{H}_{t} & =\mathbf{V}+\mathbf{A} \odot \mathbf{H}_{t}+\mathbf{B} \odot \boldsymbol{\xi}_{t-1} \boldsymbol{\xi}_{t-1}^{\prime},
\end{aligned}
$$


where $\odot$ denotes the Hadamard product ${ }^{1}$. This restriction only allows the $(i, j)$ th element in $\left\{\begin{array}{lll}\boldsymbol{\xi}_{t} & \boldsymbol{\xi}_{t}{ }_{1}\end{array}\right\}$ and $\left\{\begin{array}{lll}\mathbf{H}_{t} & 1\end{array}\right\}$ to influence the corresponding $(i, j)$ th element in $\mathbf{H}_{t}$ and can be interpreted as providing a filtered estimate of the variances and covariances.. The restriction is quite plausible and has the desired effect of reducing the number of parameters to be estimated. Compared to the most general formulation, the V, A and B matrices each have $\frac{1}{2} n(n+1)$ parameters for estimation. For models with $n=3$ and $n=5$ now have only 18 and 45 parameters respectively.

Another parameterisation termed the Baba, Engle, Kraft and Kroner, $\mathrm{BEKK}$, representation involves the equivalent number of parameters for estimation in the $\mathrm{GARCH}(1,1)$ model but has the advantage that it guarantees that the estimated time-varying variance-covariance matrices are positive definite. This model was first used in Engle and Kroner(1993) and may be expressed as follows:

$$
\mathbf{r}_{t}=\alpha+\boldsymbol{\xi}_{t}
$$

\footnotetext{
${ }^{1}$ The Hadamard product of two $N x N$ matrices $X$ and $Y$ is defined as;

$$
\begin{aligned}
X \odot Y & =\left[\begin{array}{ll}
x_{11} & x_{12} \\
x_{21} & x_{22}
\end{array}\right] \odot\left[\begin{array}{ll}
y_{11} & y_{12} \\
y_{21} & y_{22}
\end{array}\right] \\
& =\left[\begin{array}{ll}
x_{11} y_{11} & x_{12} y_{12} \\
x_{21} y_{21} & x_{22} y_{22}
\end{array}\right]
\end{aligned}
$$
}




$$
\begin{aligned}
\boldsymbol{\xi}_{t} & \mid \Psi_{t 1} \sim N\left(0, \mathbf{H}_{t}\right) \\
\mathbf{H}_{t} & =\mathbf{V}^{\prime} \mathbf{V}+\mathbf{A}^{\prime} \mathbf{H}_{t}{ }_{1} \mathbf{A}+\mathbf{B}^{\prime} \boldsymbol{\xi}_{t-1} \boldsymbol{\xi}_{t-1}^{\prime} \mathbf{B}
\end{aligned}
$$

\subsubsection{Constant Conditional Correlations}

Bollerslev(1990) proposed an even more parsimonious parameterisation of the multivariate (G)ARCH structure. He suggested a model where the time varying conditional variances and covariances are proportional to the product of the corresponding conditional standard deviations. As the name suggests, the conditional correlation matrix is assumed to be constant, giving rise to the following model:

$$
\begin{aligned}
\mathbf{r}_{t} & =\boldsymbol{\alpha}+\boldsymbol{\xi}_{t} \\
\boldsymbol{\xi}_{t} & \mid \Psi_{t 1} \sim N\left(0, \mathbf{H}_{t}\right) \\
\mathbf{H}_{t} & =\mathbf{D}_{t}^{1 / 2} \Gamma \mathbf{D}_{t}^{1 / 2}
\end{aligned}
$$

In particular, the $\mathbf{D}$ matrix is a diagonal matrix with the conditional variances along the main diagonal and zeros elsewhere. $\boldsymbol{\Gamma}$ denotes the conditional correlation matrix and is time invariant. Consequently, all the time variation in the conditional variance-covariance matrix results from variation in the conditional variances, further reducing the number of parameters 
to estimate and the computational burden. Furthermore, it is easy to impose symmetry and once again the resulting matrices are guaranteed to be positive definite.

The validity of the assumption of constant conditional correlations is an empirical issue. However, a number of studies have shown it to be a reasonable working hypothesis e.g. Baillie and Bollerslev(1990), Schwert and Seguin(1990), McCurdy and Morgan(1991) and $\mathrm{Ng}(1991)$. This view is contested by Bera and Roh(1991) who suggest a test of the constant correlation hypothesis and find this assumption is rejected for many financial time series.

\subsubsection{Factor ARCH Models}

In an attempt to exploit the fact that the variances and covariances of financial variables may be potentially driven by a small number of common factors, Engle(1987) proposed a Factor ARCH model. One obvious appeal of this model is that it is immediately recognisable as an APT model in the conditional mean equation, where the $n x 1$ vector of asset returns, $\mathbf{r}_{t}$ is determined by a $k x 1$ vector of economic factors and a $n x k$ matrix of factor loadings. If the factors and the error term are uncorrelated (or have constant correlations), then we can write the model as follows: 


$$
\begin{aligned}
\mathbf{r}_{t} & =\mathbf{L} f_{t}+\boldsymbol{\varepsilon}_{t} \\
\boldsymbol{\varepsilon}_{t} & \mid \Psi_{t \quad 1} \sim N\left(0, \mathbf{H}_{t}\right) \\
\mathbf{H}_{t} & =\mathbf{\Phi}+\mathbf{B} \boldsymbol{\Lambda}_{t} \mathbf{B}^{\prime}
\end{aligned}
$$

where the idiosyncratic shocks, $\varepsilon_{t}$, have constant conditional covariances $\boldsymbol{\Phi}$ and the factors, $f_{t}$ have time varying conditional covariance matrices, $\Lambda_{t}$. Furthermore, if the off-diagonal elements of $\boldsymbol{\Lambda}_{t}$ are zero (or constant so that they can be combined into $\boldsymbol{\Phi}$ ) and the main diagonal contains typical elements $\lambda_{k t}$ the second order moments of the model may be written as follows:

$$
\mathbf{H}_{t}=\mathbf{\Phi}+\sum_{k} \beta_{k} \beta_{k}^{\prime} \lambda_{k t}
$$

Based on this formulation, the time variation of the conditional variances and covariances results in total from the $K$ factors. This model has been implemented empirically by Engle, Ng and Rothschild(1990) and Ng, Engle and Rothschild(1992) for US Treasury Bills and stocks respectively.

Diebold and Nerlove(1989) proposed a very similar latent variable model using factor variances not conditioned on the past information set to generate the time varying conditional second order moments. In particular, the 
variance-covariance matrix was generated by

$$
\mathbf{H}_{t}=\boldsymbol{\Phi}+\sum_{k} \beta_{k} \beta_{k}^{\prime} \phi_{k t}^{2}
$$

where $\phi_{k t}^{2}$ represent the factor variances.

\subsection{A New Representation}

Despite the wealth of parameterisations for the multivariate GARCH structure, we developed another representation of the problem. This formulation seeks to retain the parsimony of the other representations while simultaneously offering the user additional potentially important information that is ignored by the other models. We write the conditional second order moments in error correction format thereby decomposing them into the sum of their long-run and short-run components. This allows us to assess the importance of the short run effects and determine whether or not they exert a significant influence on the overall process. In particular, we model the conditional variances and covariances as the sum of their long run values and their short run deviations about these values. This is combined with the BEKK representation in order to ensure that the resulting time varying covariance matrices are symmetric and positive definite. Our parameterisation can be written as follows: 


$$
\begin{aligned}
\mathbf{r}_{t} & =\boldsymbol{\alpha}+\boldsymbol{\xi}_{t} \\
\boldsymbol{\xi}_{t} & \mid \Psi_{t 1} \sim N\left(0, \mathbf{H}_{t}\right) \\
\mathbf{H}_{t} & =\mathbf{V}^{\prime} \mathbf{V}+\mathbf{A}^{\prime}\left(\mathbf{H}_{t 1}-\mathbf{V}^{\prime} \mathbf{V}\right) \mathbf{A}+\mathbf{B}^{\prime}\left(\boldsymbol{\xi}_{t}{ }_{1} \boldsymbol{\xi}_{t-1}^{\prime}-\mathbf{V}^{\prime} \mathbf{V}\right) \mathbf{B}
\end{aligned}
$$

This parameterisation has a number of advantages over the more general formulation. Firstly, it offers a significant reduction in the number of parameters to be estimated. Now $\mathbf{V}, \mathbf{A}$ and $\mathbf{B}$ are all $n x n$ symmetric matrices requiring the estimation of $3 x[n(n+1) / 2]$ parameters in total. Therefore in the applications of the model which follow in subsequent chapters where $n=3, n=4$ and $n=5$ the number of parameters to be estimated is reduced from 78 to 18,210 to 30 and 465 to 45 respectively.

Secondly, by formulating the conditional variance-covariance structure in this way, we get an estimate of both the long-run conditional covariance matrix together with its short-run dynamics. This is important as it allows us to decide if the short-run dynamics have a contribution to make and if they are worthy of the time and effort required to estimate them. It also allows us to isolate and identify the sources of the short-run action, i.e. which parameters are most significant in determining the deviations from the long-run value. This could be an important source of information in 
problems of a larger dimension in which the author may want to restrict the number of parameters to estimate.

Thirdly, by combining this parameterisation with a variant of the BEKK representation, we have guaranteed that the conditional variance-covariance matrix of asset excess returns is positive definite. 


\section{Chapter 4}

\section{Optimal Allocation of}

\section{Domestic Assets for the UK}

\section{Investor}

\subsection{Introduction}

The aim of this chapter is to analyse the subject of optimal allocation among major classes of UK financial assets. In particular, the question is addressed from the viewpoint of a UK investor who wishes to identify the optimal mix of risky assets in which to invest a proportion of his wealth. The investor is interested in allocating funds among four assets - three risky financial assets and one riskless asset. This analysis strives to identify the proportions 
in which the risky assets must be held in order for the investor to hold the optimal risky portfolio period by period and leaves each investor to allocate their funds between this risky portfolio and the riskless asset based on their subjective preferences. The risky assets under consideration are UK equities, UK government bonds with more than 15 years to maturity and UK government bonds with less than 5 years to maturity. In this paper, it is assumed that the investor is only interested in domestic assets and is not concerned with the opportunities presented by international portfolio diversification.

The textbook theories of portfolio management focus on the individual assets at the outset. The best known of these are the mean-variance analysis of Markowitz 1952 and the Sharpe-Lintner capital asset pricing model (CAPM). In the former, investors are assumed to minimise the riskiness of the total return on the portfolio subject to achieving a target rate of return. For example, a typical requirement of a fund manager is that the portfolio achieve, say, $1 \%$ above the market return. In the CAPM the optimal portfolio trades-off expected return and variance to maximise a typically quadratic - welfare function in mean and variance. In practice, because returns - especially equity returns - are not forecastable (they are virtually serially independent), the emphasis is on minimising the variance of the portfolio's return generally by choosing appropriately the propor- 
tions in which each asset is held in the portfolio. In making this calculation it is usually assumed that the variance-covariance matrix of returns is contant over time e.g. Grubel(1968) and Levy \& Sarnat(1970). It has been shown, however, that this matrix is not constant but time-varying, see Poterba \& Summers(1987), Bollerslev, Engle and Wooldridge(1988), Giovannini \& Jorion(1990), Thomas \& Wickens(1993), Engle, Frankel, Froot \& Rodrigues(1989) etc. In the absence of transaction costs, this implies that the optimal portfolio will need to be re-balanced each period. Unlike returns, it is possible to forecast how the covariance matrix changes through time. This suggests that the aim of tactical asset allocation should be to exploit the regularities in the covariance structure of returns with the aim of reducing risk.

The main innovation of this work is that we use a multivariate GARCH model of returns to forecast their covariances. We then use these forecasts to generate the portfolio frontier period by period and identify the optimal portfolio of risky assets by finding the point of tangency between the portfolio frontier and a line drawn from the risk free rate (the Capital Market Line). Given a target rate of return for the portfolio, the optimal proportions in which each asset should be held can be calculated. Typically this results in going short in at least one asset. Since some investors may be constrained from doing this, we also calculate the optimal proportions 
when they are constrained to be non-negative. Constrained tactical asset allocation is especially relevant for UK pension funds who are forbidden by law from going short. Cumby, Figlewski \& Hasbrouck(1994) also allow the elements of the covariance matrix to vary through time but they model each element individually rather than as one multivarite system. Though computationally burdensome, we hope to exploit efficiency gains by estimating the model as a comprehensive multivariate system.

Adopting the two-fund separation theorem, we believe that all investors, regardless of preferences, will hold a combination of only two mutual funds namely the riskless asset and the optimal portfolio of risky assets. Therefore, our aim is not to identify the final investment position of an investor but rather to identify the proportions in which the risky assets should be held. Each investor may then choose their preferred combination of these funds based on subjective preference.

The plan of the chapter is as follows. Section 4.2 , recalls the empirical model presented in Chapter 3 while section 4.3 describes the data used in the implementation of the model. In section 4.4, we present the results of the analysis while section 4.5 contains our concluding remarks. 


\subsection{Empirical Model}

The aim of this work is to allow the variance-covariance matrix of asset excess returns to vary over time. In order to achieve this goal, we decided to employ a multivariate GARCH model. Such models are ideally suited to this analysis as not only are they capable of modelling the time variation, but it also captures many of the stylised facts of asset returns such as thick tails and volatility clustering. The problems of implementing multivariate GARCH models are already documented in chapter 3 so we briefly recall the new parameterisation of the model (equation (3.11)) presented earlier:

$$
\begin{aligned}
\mathbf{r}_{t} & =\boldsymbol{\alpha}+\boldsymbol{\xi}_{t} \\
\boldsymbol{\xi}_{t} & \mid \Psi_{t 1} \sim N\left(0, \mathbf{H}_{t}\right) \\
\mathbf{H}_{t} & =\mathbf{V}^{\prime} \mathbf{V}+\mathbf{A}^{\prime}\left(\mathbf{H}_{t}{ }_{1}-\mathbf{V}^{\prime} \mathbf{V}\right) \mathbf{A}+\mathbf{B}^{\prime}\left(\boldsymbol{\xi}_{t_{1}} \boldsymbol{\xi}_{t 1}^{\prime}-\mathbf{V}^{\prime} \mathbf{V}\right) \mathbf{B}
\end{aligned}
$$

where $\mathbf{r}_{t}$ in this application represents a vector of asset excess returns. Until now we have concentrated our attention on the conditional second moments of the problem and have largely ignored the conditional mean equation. When we estimate the model, we include the first lag of each variable as an explanatory variable. Furthermore, we found that it was necessary to include a dummy variable for the October ' 87 stock market 
crash as an extra right hand side variable in the conditional mean equation for UK equities. Therefore the model which we estimate is as follows:

$$
\begin{aligned}
\mathbf{r}_{t} & =\boldsymbol{\alpha}+\boldsymbol{\beta} \mathbf{r}_{t 1}+\boldsymbol{\gamma d u m} 87+\boldsymbol{\xi}_{t} \\
\boldsymbol{\xi}_{t} & \mid \Psi_{t 1} \sim N\left(0, \mathbf{H}_{t}\right) \\
\mathbf{H}_{t} & =\mathbf{V}^{\prime} \mathbf{V}+\mathbf{A}^{\prime}\left(\mathbf{H}_{t_{1}}-\mathbf{V}^{\prime} \mathbf{V}\right) \mathbf{A}+\mathbf{B}^{\prime}\left(\boldsymbol{\xi}_{t_{1}} \boldsymbol{\xi}_{t_{1}}^{\prime}-\mathbf{V}^{\prime} \mathbf{V}\right) \mathbf{B}
\end{aligned}
$$

where the vector of excess returns $r=(u k e, l b d, s b d)^{\prime}, u k e$ is the excess return of UK equities, $l b d$ is the excess return of UK government bonds with more than 15 years to maturity and $s b d$ is the excess return of UK govt bonds with less than 5 years to maturity respectively and dum 87 is a dummy variable for the October 1987 stock market crash. $\boldsymbol{\beta}$ is a $3 x 3$ matrix of regression parameters and $\gamma$ is a $3 x 1$ vector of parameters.

\subsection{Data}

This chapter uses time series data on broad classes of UK financial assets. In particular, we focus on three risky assets and one riskless asset. The risky assets used in the analysis are equities, represented by the Financial Times All Share Index; long UK government bonds represented by the FT British government stock over 15 years index; and short government bonds repre- 
sented by the FT British government stock under 5 years index. The data used in this chapter is annualised monthly total returns for each asset ${ }^{1}$. The total return data is calculated so as to take account of dividend payments in the case of equities and coupon payments in the case of government bonds. Both dividends and coupon payments are treated as if they were received in equal amounts throughout each working day of the year rather than as a lump sum at one or two distinct points in time. The rate of return on the UK government 30 day Treasury bill is taken as the risk free rate of interest available to the investor. It is true to say that this asset is riskless at least in the nominal sense. All data was sourced from DATASTREAM.

The data covers a sample period in excess of 20 years beginning in January, 1976 and finishing in February, 1997. This sample yields a total of 251 usuable observations. We have chosen to work exclusively with rates of return in excess of the risk free rate. This approach has been adopted to prevent volatility in the risk free rate from incorrectly contributing to the risk of the optimal risky portfolio. Since the risk free rate is perfectly predictable at the start of each period and therefore part of the investor's information set when the allocation decision is made, its inclusion would tend to over-estimate the total risk of the portfolio.

\footnotetext{
${ }^{1}$ All returns are nominal values. We use nominal returns to be consistent with other studies and using the results of Engle(1984) and Cumby(1988) where it is argued that both the behaviour of both nominal and real returns are substantially the same.
} 
From an econometric point of view, there is a further benefit from working with excess returns, namely that all series are stationary and do not require differencing ${ }^{2}$.

\subsection{Estimating the Model}

\subsubsection{Convergence of the Log Likelihood Function}

The model outlined earlier in 4.2 was estimated by recursively maximising the following log likelihood function using the Berndt, Hall, Hall \& Hausmann (BHHII) algorithm

$$
\log L=-\frac{n}{2} \log (2 \pi)-\frac{1}{2}\left(\log \left|\Omega_{t}\right|-u_{t}^{\prime} \Omega_{t}^{1} u_{t}\right)
$$

where $\mathrm{n}$ is the number of assets.

As with all multivariate GARCH models, one of the most important questions to address is the convergence procedure. Meeting the convergence criteria under the $\mathrm{BHHH}$ algorithm requires both stabilised parameter values as well as a stabilised function value. Choosing starting values near the optimum is vital as otherwise the models often fail to converge. The error correction structure of 4.2 is useful in this respect as it enables us to use

\footnotetext{
${ }^{2}$ A number of formal tests confirm that the series are stationary namely, Dickey Fuller, Augmented Dickey Fuller, Phillips Peron and Stock-Watson Tests.
} 
the unconditional error covariance matrix to obtain a consistent estimate of V. Initial values of the explanatory variables and error terms in the conditional mean equations can be conveniently generated by a prior Ordinary Least Squares estimation of these mean equations. These residuals are then used to estimate the unconditional variance-covariance matrix and we then obtain an initial estimate of $\mathbf{V}$ using a choleski factorisation of the resulting estimate. The $\mathbf{A}$ and $\mathbf{B}$ matrices are initialised with an arbitrary small number along the diagonal and zeros elsewhere. This procedure is crucial to the successful implementation of the model as bad starting values can often mean that the algorithm fails to meet the convergence criteria or may lead the algorithm into an area where the variance-covariance matrix is no longer positive definite, thus causing the model to fail.

\subsubsection{Results}

When the model as specified in equation (4.2) was estimated and convergence achieved, it yielded the following results. The results for each matrix are presented with the corresponding t-statistics in brackets underneath. 


\section{Conditional Mean}

$$
\boldsymbol{\alpha}=\left[\begin{array}{l}
13.39 \\
(3.75) \\
4.07 \\
(1.35) \\
0.93 \\
(0.87)
\end{array}\right], \boldsymbol{\beta}=\left[\begin{array}{ccc}
0.006 & 0.096 & 0.267 \\
(0.09) & (0.95) & (0.83) \\
0.042 & -0.016 & 0.318 \\
(0.71) & (-0.13) & (1.26) \\
-0.027 & 0.038 & 0.015 \\
(-1.20) & (1.60) & (0.179)
\end{array}\right], \boldsymbol{\gamma}=\left[\begin{array}{l}
-392.25 \\
(-4.98) \\
0
\end{array}\right]
$$

Conditional Covariances

$\mathbf{V}, \mathbf{A}$ and $\mathbf{B}$ are all symmetric matrices.

$$
\mathbf{V}=\left[\begin{array}{lll}
57.37 & & \\
(22.58) & & \\
23.15 & 30.72 & \\
(6.94) & (1.99) & \\
8.72 & 6.81 & 9.06 \\
(7.02) & (1.77) & (7.33)
\end{array}\right]
$$




$$
\mathbf{A}=\left[\begin{array}{lll}
0.82 & & \\
(3.44) & & \\
0.09 & 0.90 & \\
(0.12) & (7.40) & \\
0.29 & 0.22 & 0.31 \\
(1.37) & (1.11) & (1.5)
\end{array}\right], \mathbf{B}=\left[\begin{array}{lll}
0.06 & & \\
(1.02) & & \\
0.03 & 0.08 & \\
(0.60) & (1.20) & \\
0.11 & 0.02 & 0.16 \\
(4.62) & (0.40) & (1.10)
\end{array}\right]
$$

\subsubsection{Estimates of the Conditional Mean}

The lack of significance in the estimates of $\boldsymbol{\beta}$ are consistent with the usual finding of the virtual serial uncorrelatedness of total stock and bond returns. Consequently, while we retain the mean specification for estimation purposes, we use the vector of historical asset means to generate the portfolio frontiers. It has the added advantage that all of the variation in the estimated frontiers, and hence the portfolio shares, can be attributed to variation in the conditional covariance matrix of excess returns.

This is also the assumption made by Cumby, Figlewski and Hasbrouck(1994) who also use the historical mean of each asset as its expected value. Jobson \& Korkie(1981) advocate the use of global shrinkage based on Stein estimators whereby all assets of the same class have the same expected excess return. This is an extreme case of Stein estimation with the individual as- 
set being assigned a weight of zero and the global mean having a weight of one. Jobson \& Korkie show that this approach significantly improved the practical application of the Mean-Variance framework. Since we are working with financial asset indices as opposed to individual securities, both of these appoaches reduce to the same thing.

Another reason for making this assumption is that the sensitivity of the portfolio shares to small variations in the mean is far greater than that to variations in the covariance matrix, Kallberg and Ziemba(1984). Best and Grauer(1991) show that even small changes in the mean vector can result in dramatic variation in the composition of the estimated optimal portfolio of risky assets.

Continuous re-balancing of the portfolio to changes in the predicted excess return would not only be expensive due to transaction costs, it would also be counter-productive because of the lack of persistence of the deviations of excess returns from their unconditional means. This is not true of the re-balancing due to changes in the conditional variance because of their much higher degree of persistence and their lower volatility.

\subsubsection{Estimates of the Conditional Covariance Matrix}

The estimates of the elements of $\mathrm{V}$ are all significant at the $10 \%$ level and all but one are significant at the $5 \%$ level. Although, many of the elements of $\mathbf{A}$ 
and $\mathbf{B}$ are not significant, even at the $10 \%$ level, sufficient are significant to show that there are deviations of the short-run from the long-run covariance matrix. In the main, these are due to autocorrelation in the conditional variances, but there is also a significant effect arising from the interaction between the excess returns on equity and short-term government bonds.

Roughly speaking and ignoring the other elements, the greater the elements on the leading diagonals of $\mathbf{A}$ and $\mathbf{B}$, the more the conditional covariance matrix deviates from the long-run value. The more significant these elements the more predictable are these deviations. The estimates suggest that the deviations are both persistent and predictable. Figure 4-1 plots the conditional and unconditional variances from the three excess returns. The deviations from the long run are most persistent for the long government bond. It is also clear that equities have predictable and persistent deviations, most notably in 1983-4 and since 1993. These are precisely the conditions in which there is greatest benefit to taking account of a time-varying covariance matrix of excess returns in determining asset allocation.

\subsubsection{Frontier Movements}

Apart from changes to the target rate of return, variations in the optimal portfolio weights are due entirely to movements in the portfolio frontier 
brought about by new information on next period's conditional covariance matrix. This new information is the cause of the time variation in the conditional covariance matrix. Some idea of the extent of the movements in the frontier within the sample period can be obtained from Figures 4-24-6. The position of the frontiers reflect the minimum portfolio standard deviation for a given portfolio return, hence this is just another way of comparing portfolio standard deviations. Figure 4-2 shows how the frontier has moved over time by displaying the frontier in September at four year intervals from 1976-96. Figure 4-3 provides information on the distribution of the frontiers since it displays the minimum, maximum, mean and median frontiers for the whole sample. The global minimum variance portfolio of the frontier was calculated for each monthly period of the analysis and these portfolios were used to compute the frontiers depicted in this figure. It reveals that the distribution is highly positively skewed with a long tail to the right. The standard deviations of the minimum variance portfolios range from a minimum of approximately $8 \%$ in February 1996 to a maximum of $28 \%$ in September 1981. The skewness obviously has major implications for the choice of optimal portfolio. Assuming the same frontier for each period by taking the mean or the median frontier would have seriously underestimated the riskiness of the assets in September 1981. Figure 4-4 examines the last six months of 1981 in more detail. It shows how volatile 
the frontier can be over a short time horizon.

A very revealing comparison is between the frontiers based on constant covariance matrices computed from both a simple OLS estimate of the unconditional covariance matrix and the long-run matrix $\left(\mathbf{V}^{\prime} \mathbf{V}\right)$ of our model and the frontiers obtained from using a time-varying conditional covariance matrix. In Figure 4-5 we include the frontiers generated by the OLS estimate, the long-run unconditional covariance matrix and the mean and median of the conditional covariance matrices. Both the frontiers associated with the unconditional covariance matrices lie further from the origin than their time-varying counterparts. This shows the considerable reduction in riskiness of the portfolio that can be achieved by using the conditional covariance matrix instead of a constant, unconditional, covariance matrix. The frontier generated by the OLS estimate is to the right of the others, demonstrating that such a simple estimate tends to overestimate the riskiness of the assets and that even in using our model only to estimate the unconditional covariance matrix, there are risk reduction gains to be exploited over using the more simple OLS approach.

Finally, we examine the consequences of allowing the conditional mean to be time varying by failing to omit the insignificant terms in the conditional mean in the above calculations. The means and medians of the frontiers computed from including and excluding the lagged dependent variable from 
the model (and then re-estimating the model) are shown in Figure 4-6. We find that distribution of the frontiers for the model that includes the lagged excess returns in the model lies to the left of the distribution of the frontiers that restricts the model by excluding these lags. Thus, even if the dynamics in the conditional mean are imprecisely estimated, including them in the model results in a substantial reduction in portfolio risk.

\subsubsection{Optimal Portfolios}

\section{Unrestricted Weights}

Having generated the portfolio frontiers, we now proceed to identify the optimal portfolio in each period. This is achieved by finding the point of tangency between the portfolio frontier and a line drawn from the rate of return on the riskless asset. Since we are working exclusively with excess returns, this line will now go through the origin. It can be shown that for every period, the tangential line will have a slope, $\mathrm{m}$, given by (see Appendix 1):

$$
m=\frac{c_{t} R_{p t}-b_{t}}{\sqrt{\left(a_{t}-2 b_{t} R_{p t}+c_{t} R_{p t}^{2}\right)\left(a_{t} c_{t}-b_{t}^{2}\right)}}
$$

The point of intersection identifies the optimal portfolio of risky assets which will have an excess return $=a_{t} / b_{t}$ (Appendix 1$)$. All of these variables are defined as in section 2.2. Figure 4-7 shows how both the expected excess 
return and the standard deviation of the optimal risky portfolio changed over time. As we would expect, there is a clear direct relationship between the excess return and the standard deviation. Also the standard deviation is much more volatile than the excess return. Table 4.1 summarises the key features of the optimal portfolios.

\begin{tabular}{|l|l|l|l|}
\hline & Mean & Max. & Min. \\
\hline Excess Return & 7.41 & 16.1 & 4.34 \\
\hline Standard Deviation & 46.0 & 102.3 & 26.8 \\
\hline
\end{tabular}

Table 4.1: Key features of optimal domestic portfolio

We begin the analysis by computing the optimal asset proportions for a buy and hold portfolio generated by both our estimates of a constant unconditional covariance matrix. The asset holdings are shown in table 4.2.

\begin{tabular}{|l|c|c|c|}
\hline & Equity & Long Bond & Short Bond \\
\hline OLS estimate & $71.3 \%$ & $18.2 \%$ & $10.5 \%$ \\
\hline Long-run Matrix & $69.6 \%$ & $26.2 \%$ & $4.2 \%$ \\
\hline
\end{tabular}

Table 4.2: Optimal buy and hold portfolios

The two portfolios differ greatly, especially in the importance attached to the government bonds. The portfolio based on the OLS estimate has a large equity holding which is consistent with it producing the most risky portfolio frontier in figure $4-5$. 
With the optimal portfolios located and identified for each period, we calculate the proportions in which each of the risky assets must be held in order for the investor to hold this portfolio. We calculate the weight vector according to equation 2.17. This calculation places no restriction on the sign of the weights and it is therefore possible for the investor to take a short position in an asset, thus allowing the investor to invest more than $100 \%$ of investable funds in the other assets. Figure $4-8$ shows the weights given to each asset over the sample. It is clear from this picture that the optimal portfolio often involves taking a short position in the shorter UK government bond, especially in the earlier part of the sample, allowing a stronger position in the relatively higher return assets. As expected, equities are the dominant asset, accounting for $70 \%$ of the portfolio on average. The weight given to equity is consistently positive and indeed never falls below $38 \%$, while on a number of occasions more than $100 \%$ of funds are invested in equities. The government bonds, on the other hand, enjoy varying degrees of popularity. On average, the longer bond and the shorter bond account for $20 \%$ and $10 \%$ of the portfolio respectively. The longer bond is only held short in one period out of the 251 period sample while the holdings of the shorter UK government bond is the most volatile of all assets. Its weight in the optimal portfolio undergoes large changes from period to period. Table 4.3 captures the main statistics of the weights given to each asset in the 
portfolio.

\begin{tabular}{|l|c|c|c|}
\hline & Mean Weight & Maximum & Minimum. \\
\hline Equities & $69.7 \%$ & $160 \%$ & $38.1 \%$ \\
\hline Long Bond & $19.8 \%$ & $84 \%$ & $-3.6 \%$ \\
\hline Short Bond & $10.5 \%$ & $65 \%$ & $-144 \%$ \\
\hline
\end{tabular}

Table 4.3: Summary statistics for optimal unrestricted domestic portfolio

Two practical considerations suggest that this may not always be an attractive or viable asset allocation strategy. Firstly, these calculations ignore the transactions costs of continuously rebalancing the portfolio. Given the volatility of the shares, this could be considerable and may act as a deterent to implementing this investment strategy. Secondly, many investors are precluded from going short either by choice or by law. Mutual fund managers in the UK are prohibited by law from holding short positions. We therefore examine optimal asset allocation subject to a non-negativity constraint on asset shares.

\section{Restricted weights}

Although it is not possible to provide a closed-form expression for the portfolio shares when a non-negativity constraint is imposed, they can be obtained for each period using Quadratic Programming. ${ }^{3}$ Instead of solving for the

\footnotetext{
${ }^{3}$ See Fletcher(1981) for a discussion of Quadratic Programming techniques.
} 
mean return for the optimal portfolio as above, it is now necessary to specify a target rate of return. We choose the target return to be the average return on the unrestricted optimal portfolio. This implies that, in terms of the mean portfolio return, investors are not penalised by the restriction, and it aids comparisons with the unrestricted case.

The restricted shares as well as the long-run asset proportions are displayed in Figure 4-9, and summary statistics are reported in Table 4.4. The main change compared with the unrestricted shares is the much lower variation in the shares. Their mean values are hardly altered. For equities the share now ranges between $62 \%$ and $72 \%$ of the portfolio compared with $38 \%$ to $160 \%$ previously. The shares of the two types of government bonds are almost a mirror image of each other, and their range of variation is dramatically reduced. This indicates that most of the portfolio rebalancing is between longer-dated and shorter-dated government bonds.

\begin{tabular}{|c|c|c|c|}
\hline & Mean Weight & Maximum & Minimum \\
\hline Equities & $69.4 \%$ & $72.8 \%$ & $62.7 \%$ \\
\hline Long Bond & $20.3 \%$ & $37.3 \%$ & $11.5 \%$ \\
\hline Short Bond & $10.3 \%$ & $15.6 \%$ & $0 \%$ \\
\hline
\end{tabular}

Table 4.4: Summary statistics for optimal restricted domestic portfolio

A quick, yet informative, check on the validity of this approach is to compare the actual performances of the constrained time-varying portfolio 
with a more traditional buy and hold portfolio. The asset proportions of the latter being determined by the unconditional covariance matrix. Since our goal is to minimise risk, we would expect that the variance of the continuously re-balanced portfolio should not be greater than the variance of the other. We would also hope that the returns would not be significantly different. Figure 4-10 supports our hypothesis. The top panel of the graph shows the ratio of the return on the time-varying portfolio to the return on the buy and hold portfolio. The ratio is usually very close to unity and shows that neither portfolio consistently outperforms the other. The lower panel plots the ratio of the variances. Now we see that our tactical asset allocation strategy systematically delivers lower risk than the more conventional portfolio. The risk reduction is of the order of $5 \%$ - in the world of investment where even the slightest advantage can mean massive financial rewards, this reduction is very substantial and highly significant.

Of course, we must remind ourselves at this stage that the portfolios identified thus far contain only risky assets and are not meant to define the total investment position of the individual. Each individual should hold a combination of the risky portfolio and the riskless asset. This decision depends on the individual preferences of each investor. If an individual's preference is to bear less risk than that associated with the risky portfolio, then they should allocate total investable funds between the riskless asset 
and the risky mutual fund, holding a positive amount of both funds. Likewise, if an investor is willing to bear more risk than the risky portfolio, they should take a short position in the riskless asset and invest all their own funds plus the extra money generated by this short position in the risky portfolio. Whatever the preferences of the individual, total funds can be allocated between these two mutual funds. This analysis does not indicate the final investment position of any investor but it identifies the two mutual funds between which resources should be allocated so as to maximise the return to the investor.

\subsection{Conclusion}

The aim of this chapter was to identify the time-varying optimal portfolio of risky assets in which a UK investor should invest. We focus on an investor who decides to invest in three domestic assets. The chapter uses the portfolio theory of Markowitz(1952) as its foundation and then tries to incorporate a more realistic specification of the risk associated with asset allocation. The main innovation of the chapter is that the covariance matrix of asset excess returns, and therefore the portfolio frontier, is allowed to vary in each period of the analysis. This time variation in the conditional second order moments is captured by a multivariate $\operatorname{GARCH}(1,1)$ model.

We specified a model that has a first order VAR structure in the con- 
ditional mean equation, with the conditional second moments following a $\operatorname{GARCH}(1,1)$ process. This enables the elements of the conditional covariance matrix to vary over time. The chapter applies the new parameterisation of the multivariate GARCH structure outlined in chapter 3. This parsimonious representation writes the conditional second order moments in error correction format thus enabling us to distinguish between long-run and short-run sources of financial asset volatility. Combining this with the BEKK representation, we ensure that the time-varying conditional covariance matrices generated by the model will be positive definite. It also allows for easy assessment of the importance of the short-run dynamics.

The key results of the model are that the elements of the conditional covariance matrix of asset returns are highly variable over time. Consequently, the portfolio frontiers also exhibit a great deal of time variation. It can be seen that the portfolio frontier changes in both shape and location throughout the 20 year sample. The results also emphasise the importance of the short-run dynamics of the model with a great deal of persistence in evidence in the second order moments of the process. We provide graphical evidence that the UK investor can reduce portfolio risk by using conditonal second order moments as opposed to more traditional static estimates.

Having located and identified the portfolio frontier in each period, we found the proportions in which the three risky assets should be held in order 
for the investor to hold this optimal portfolio of risky assets. UK equities were found to be the dominant asset throughout the sample, accounting for $70 \%$ of the portfolio on average. The long UK government bond was also held consistently in the portfolio with an average weighting of $20 \%$. The shorter UK government bond holding was more volatile and was actually held short on many occasions with the extra funds generated by this position invested in equities. Its mean weighting in the portfolio was $10 \%$. This led to often dramatic alterations in the composition of the optimal portfolio. A more practical strategy was then adopted with the weights given to each asset restricted to be non-negative. This is equivalent to prohibiting short sales, a restriction enforced on UK fund managers by law. With this restriction in place, the proportion of funds allocated to each asset is much less volatile. The mean positions are unaltered but the range of their movements is much smaller. This is a very pleasing feature for an investment manager operating in a world with transaction costs. Therefore, enforcing the non-negativity restriction on the portfolio asset weights yields a much more practical invest ment strategy.

Crucially, our approach delivered its aim of portfolio risk reduction. We found that the re-balanced portfolio systematically outperformed its buy and hold counterpart in terms of lower risk - $5 \%$ on average - while achieving excess returns that were predominantly the same. The evidence sug- 
gests that significant risk reduction benefits can be reaped from utilising conditional second order moments as opposed to the more traditional unconditional estimates. 
Figure 4.1

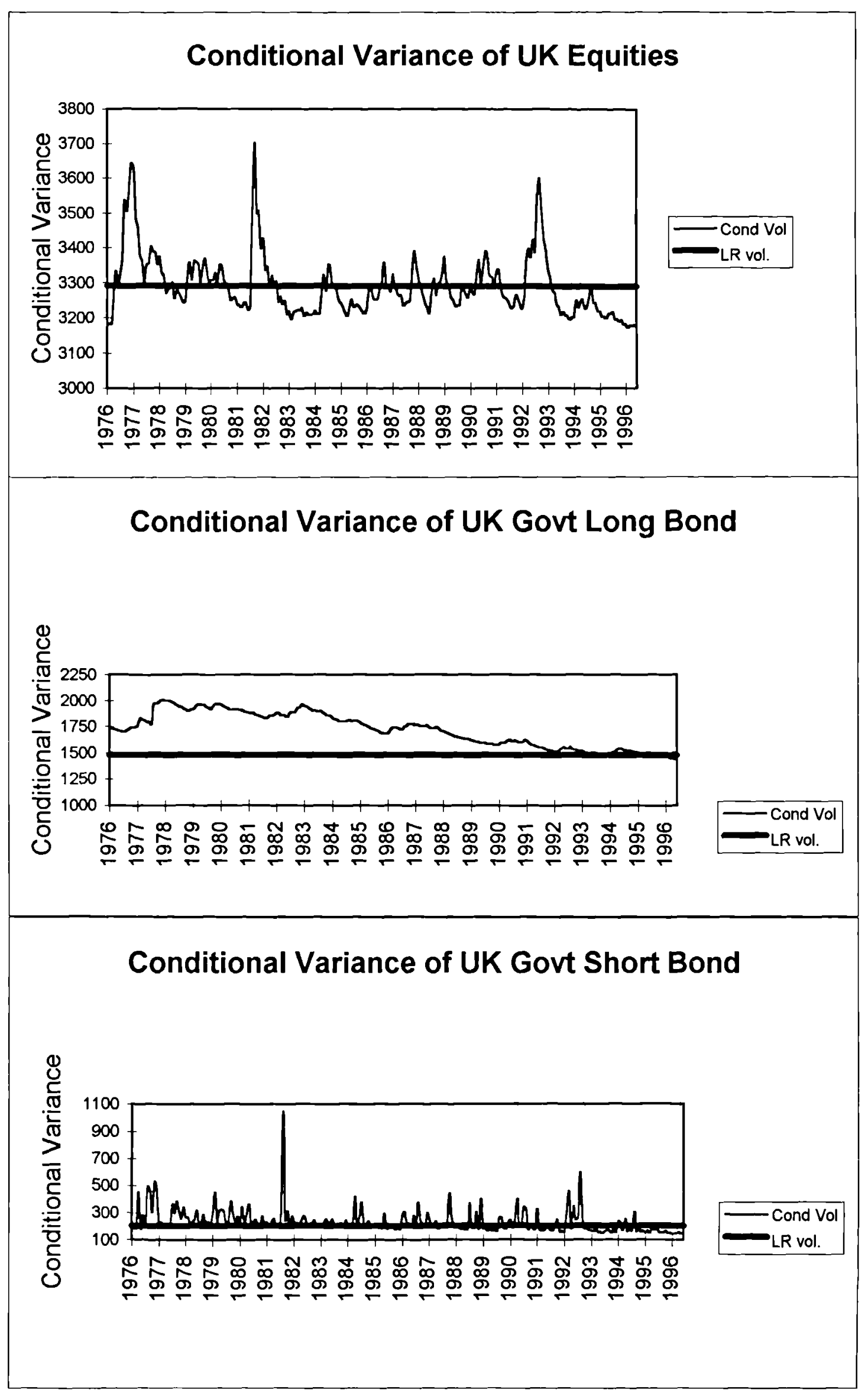


Figure 4.2

Movement of Portfolio Frontier: 4 year intervals

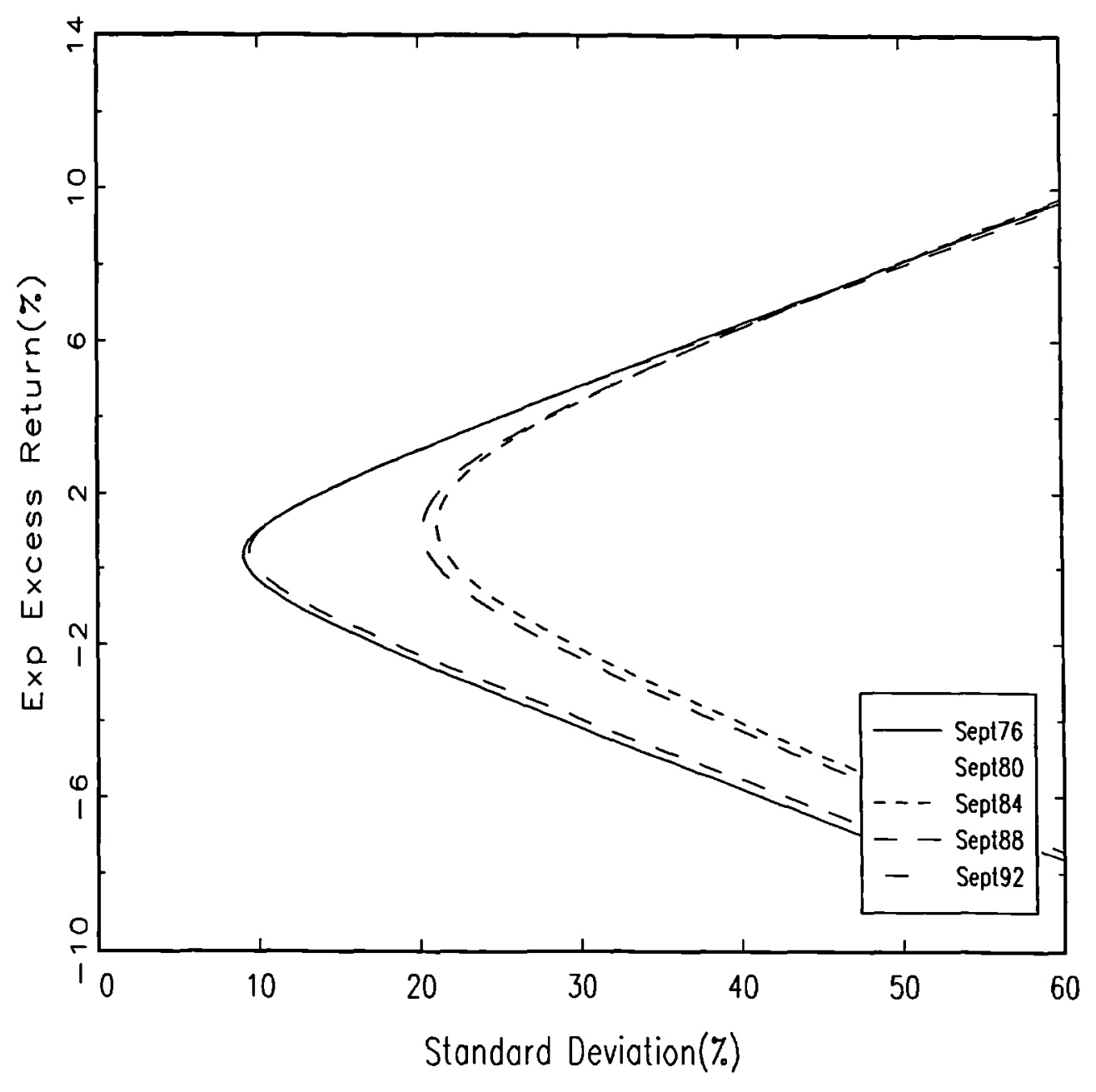




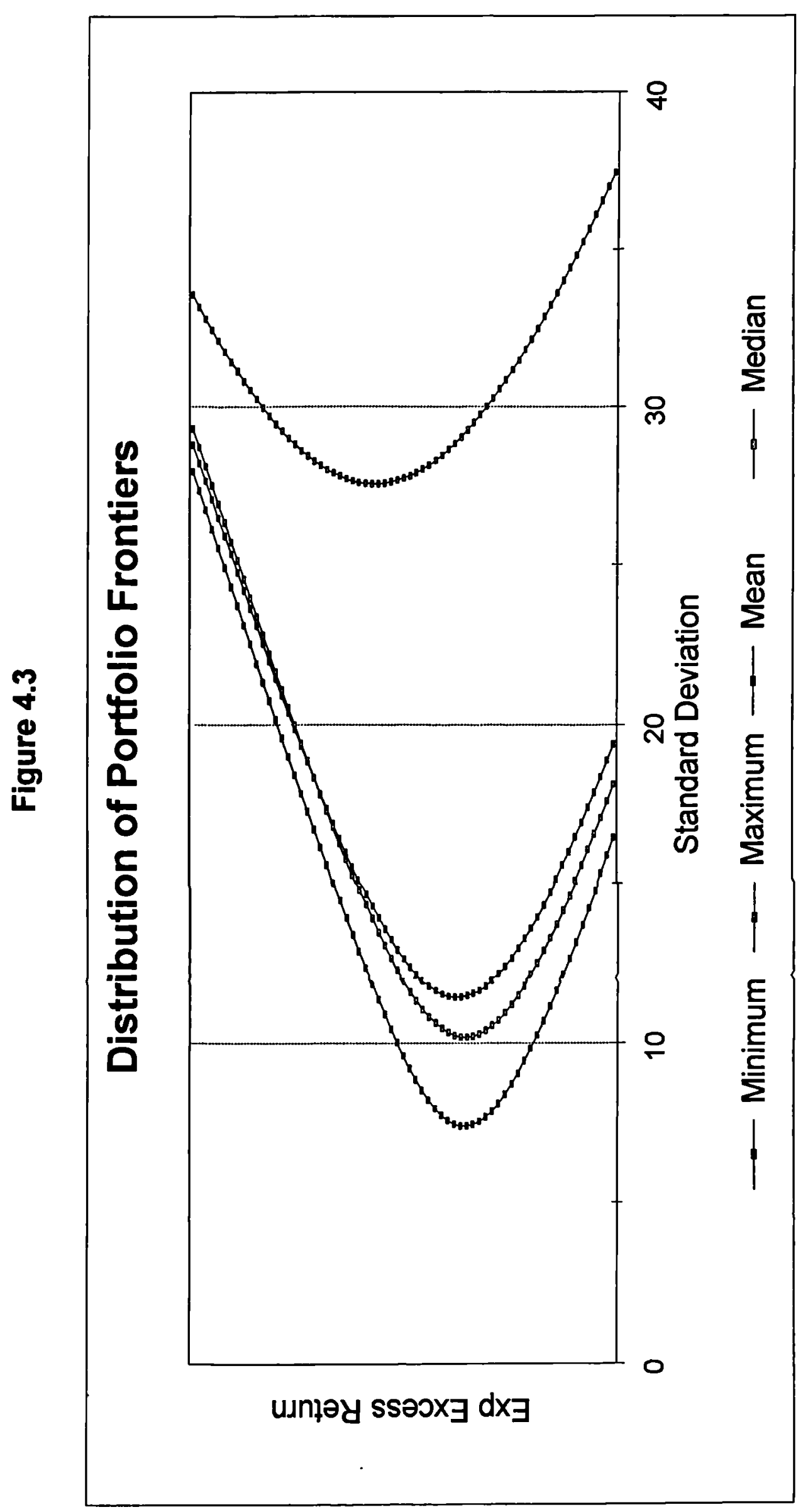

œ 
Figure 4.4

Movement of Portfolio Frontier: July - Dec 1981

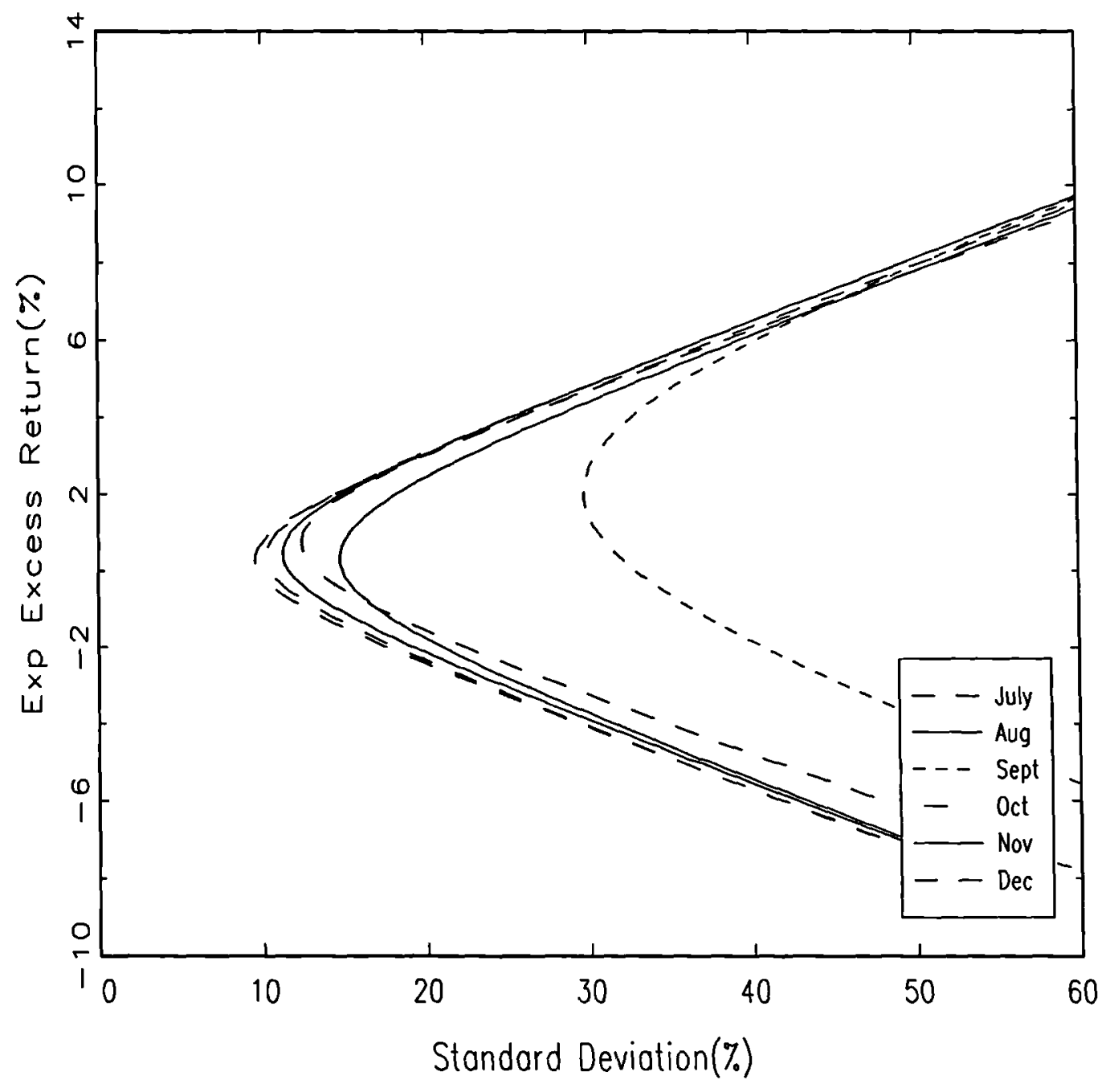




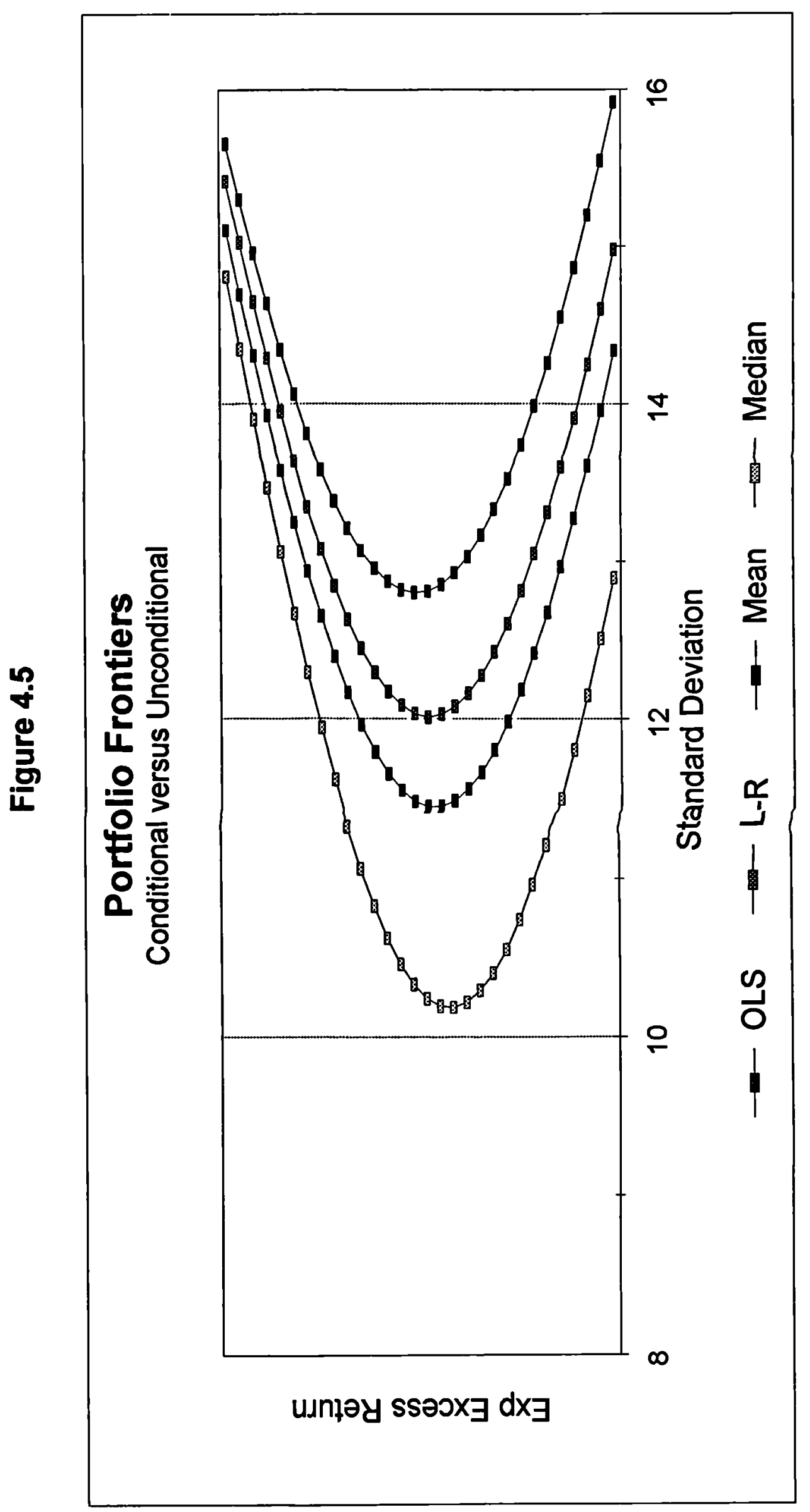

$\mathscr{\infty}$ 


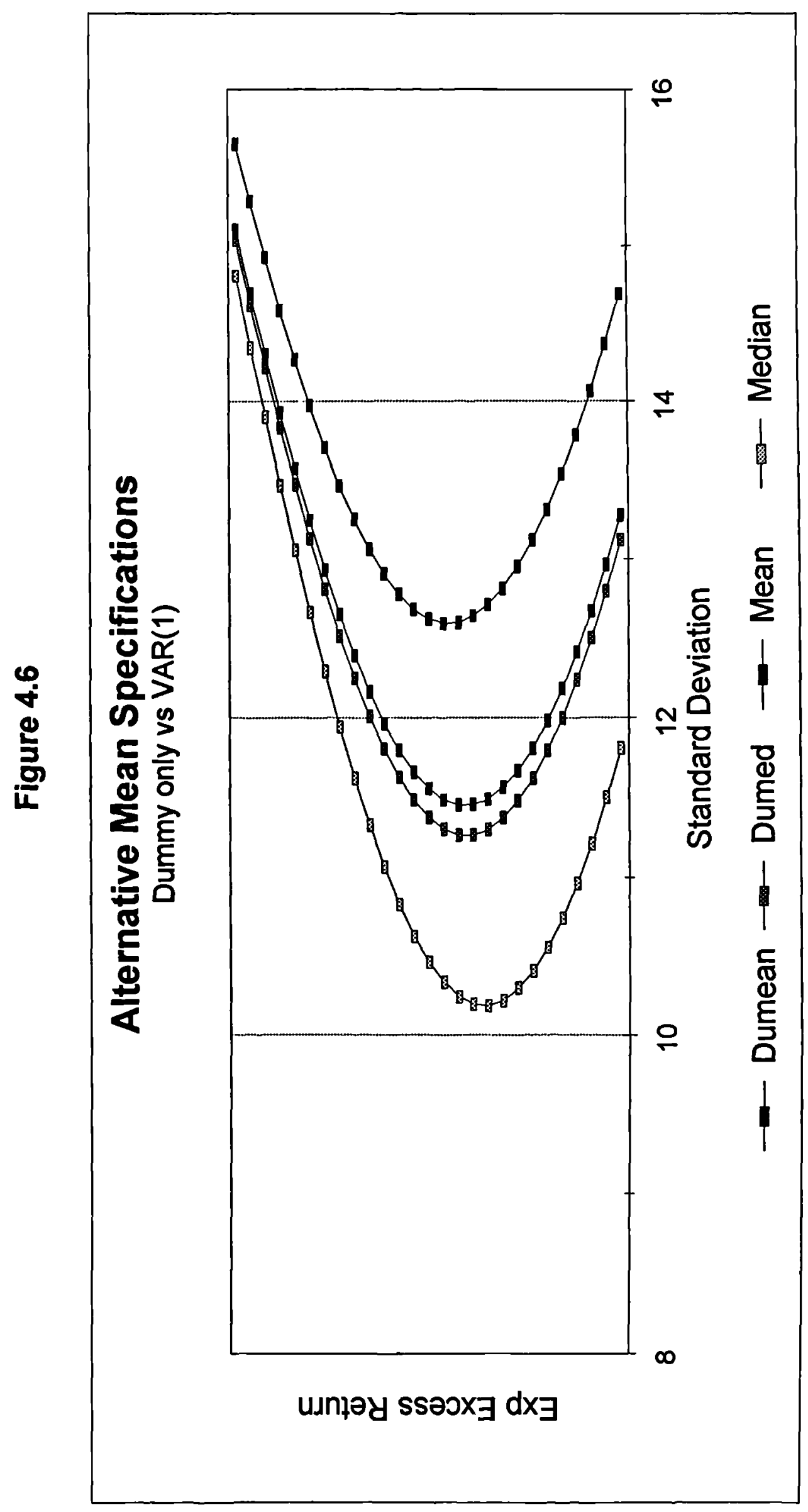

$œ$ 
Figure 4.7

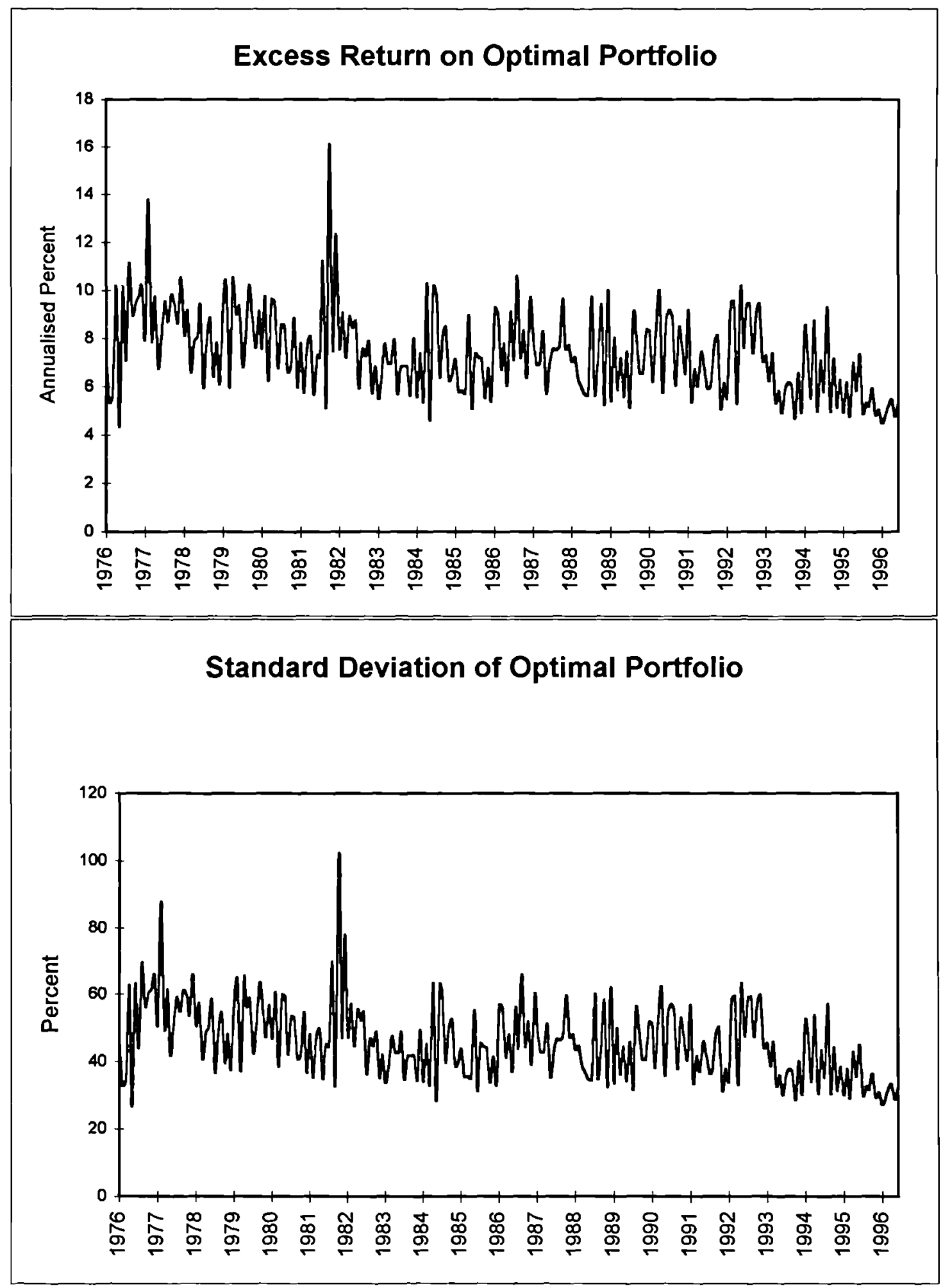



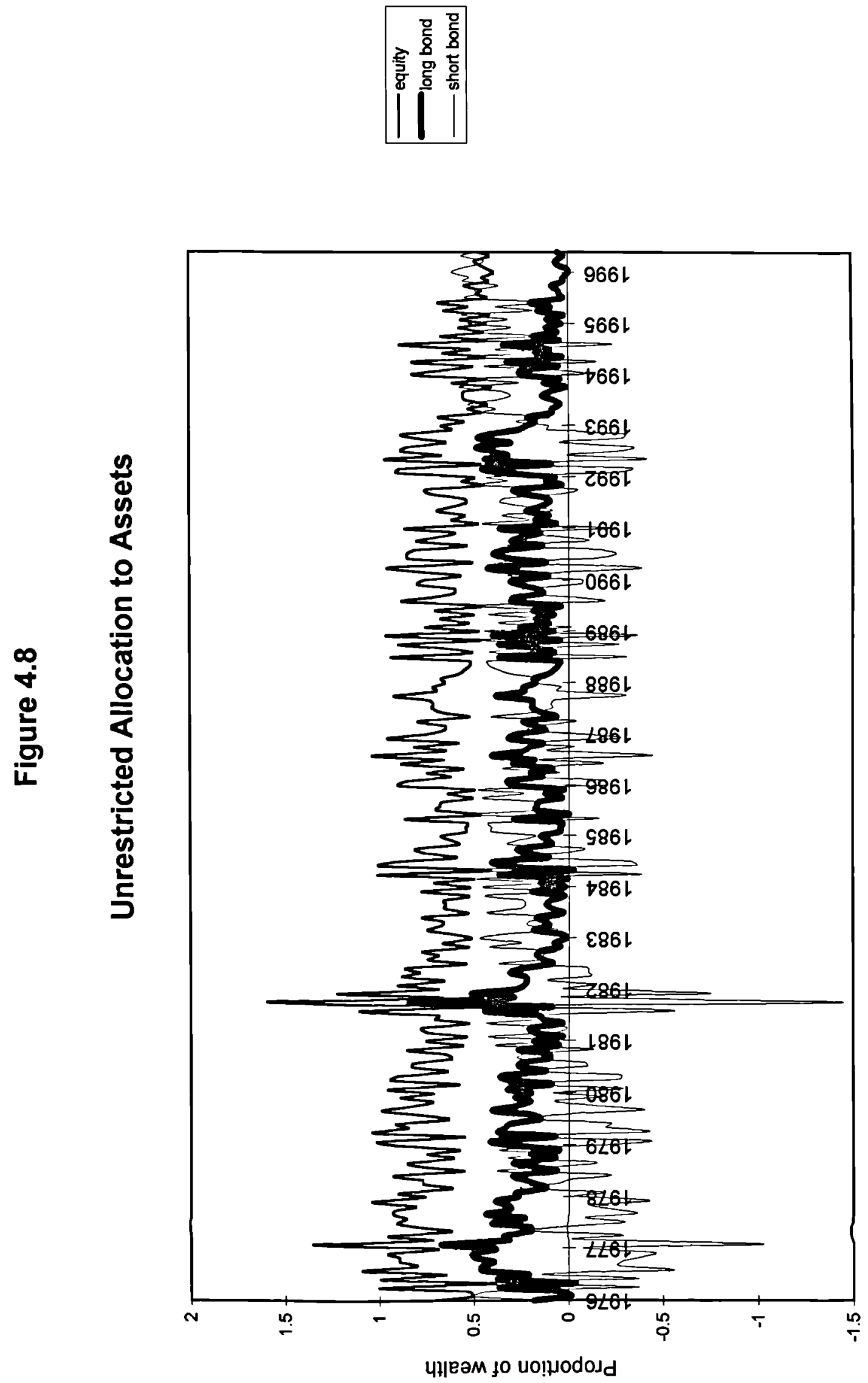

$\infty$ 


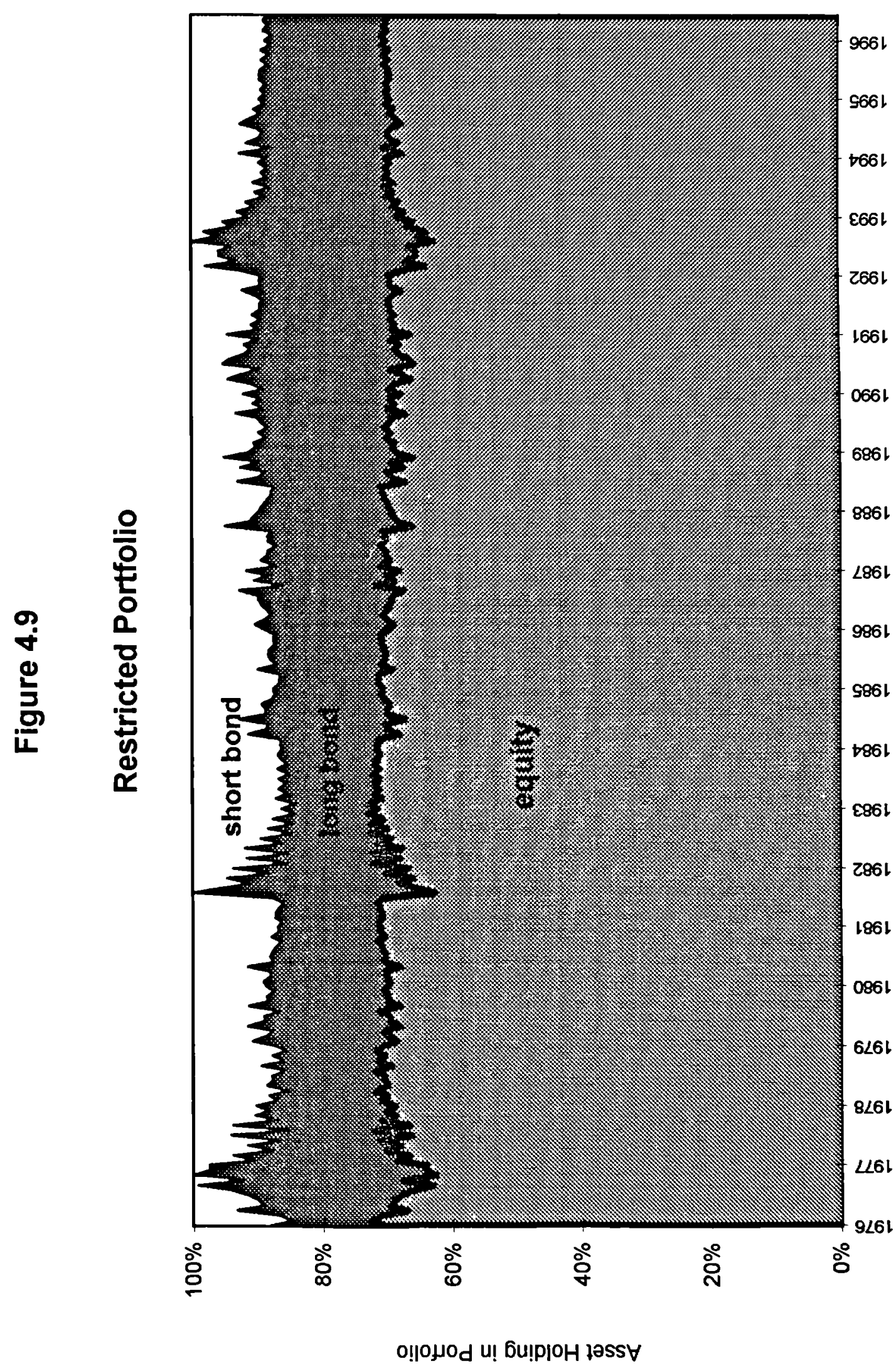


Figure 4.10

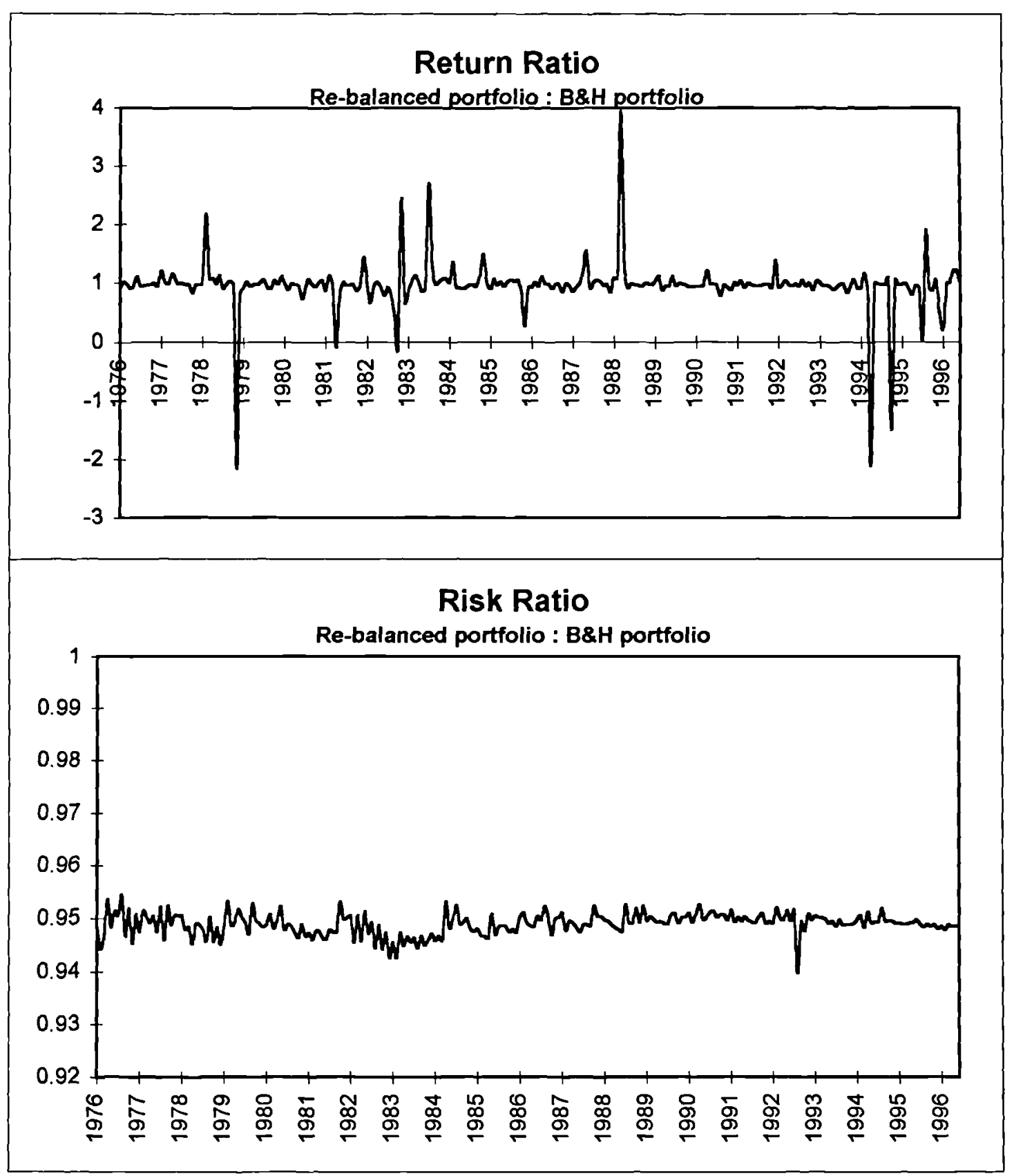




\section{Chapter 5}

\section{Optimal Asset Allocation with}

\section{an International Perspective.}

\section{Is Investor Location}

\section{Important?}

\subsection{Introduction}

This chapter is concerned with the potential benefits of holding an internationally diversified portfolio that is re-balanced each period to take account of time-variation in the covariance matrix of returns. This question is addressed from both the perspective of UK and US investors and we also inves- 
tigate the importance of location in the optimal asset allocation decision. In particular, we contrast the optimal portfolio of risky assets that should be held by an investor in the UK and the US when both are presented with the opportunity of investing in UK and US equity and a domestic government bond. Empirical research suggests that diversification across international markets is more important than diversification across industries, see Heston and Geert Rouwenhorst(1995). This analysis allows us to address the question of home bias and quantify if this remains a puzzle.

From the very early literature, there has been much evidence that large benefits are available to investors who diversify their portfolio to hold foreign assets. Grubel(1968) and Levy and Sarnat(1970) were among the first studies to reach such a conclusion. More recent studies are equally supportive of diversification, especially Grauer and Hakansson(1987) whose results confirm that US investors can reap "remarkably large" gains from including non-US assets in their portfolio of risky assets. Based on the paired $t$-test, these internationally diversified portfolios realised returns that were significantly higher than those generated by a portfolio consisting entirely of domestic stocks. Furthermore, the gains increased as the investor became more risk averse. De Santis and Gerard(1997) provide evidence that even though equity market declines are contagious across countries, US investors may still earn expected gains of $2.1 \%$ on average from holding foreign stocks 
and despite increased financial market intergration, these ex-ante gains have not fallen. Eun and Resnick(1988) and Jorion(1985) both show that hedging foreign exchange risk can potentially increase the gains from international diversification. While many of these studies concentrated on equity markets, Levy and Lerman(1988) find that a US investor who diversified across world bond markets could have realised returns more than twice the mean rate of return on a domestic US bond portfolio at the same risk level. Therefore, it seems that investors should seize the opportunity to diversify across markets. By including both equity and bonds and taking account of the time-variation in returns we expect to find that gains from international diversification are even greater than previously found.

This chapter is based on the portfolio selection theory of Markowitz(1959) but differs from earlier work in that the variance-covariance matrix of asset excess returns is allowed to vary through time. The study locates the optimal portfolio for each monthly period of the analysis and identifies the optimal mix of risky assets for both the UK and US investor. Therefore, the solution offers the investor a strategy for tactically allocating his portfolio over time. We find that the domestic equity dominates the optimal portfolio in each period but the foreign asset is also an important constituent of the portfolio and dominates the domestic bond on average. We also observe that when we compare our results to the available surveys of asset holdings, the 
home bias puzzle is much more acute in the US. The surveys suggest that US investors hold at least $94 \%$ of their wealth in domestic assets, whereas our analysis suggests that the US investor should, on average, hold $20 \%$ of funds for investment in risky assets in UK equity. The equivalent holding of US equity for the UK investor is $25 \%$, but the survey material shows that the UK investor holds only $18 \%$ of the risky portfolio in all foreign assets.

The remainder of this chapter is structured as follows. Section 5.2 presents a survey of the literature pertaining to the home bias puzzle and reviews some of the explanations that have been put forward in an effort to reconcile the theory and the observed behaviour of investors. Section 5.3 reviews the econometric techniques employed to address the question, contains the model for estimation and discusses the data. The results of the analysis are presented in section 5.4 , while section 5.5 presents the results of our tactical allocation strategies. Section 5.6 offers a summary and concluding remarks.

\subsection{Home Bias}

The home bias or international diversification puzzle refers to the widely accepted fact that investors hold too little of their financial wealth in foreign 
assets $^{1}$. In the past, many commentators explained this phenomenon as a consequence of national barriers to capital flows and the very low levels of financial market integration across the world. However, this is no longer a valid explanation in the late 1990's with limits to foreign exchange transactions and impediments to inward investment almost totally eliminated in all of the major developed markets and technological advancements helping financial markets to become increasingly integrated. Yet French and Poterba(1991) report that US investors hold $94 \%$ of their financial wealth in domestic securities, with Japanese and UK investors holding $98 \%$ and $82 \%$ of their respective portfolios in domestic assets. Likewise Cooper and Kaplanis(1994) estimate that the percentage of domestic equities in the total equity portfolio in US, UK and Japan is $98 \%, 79 \%$ and $87 \%$ respectively.

A vast literature has been produced in an effort to explain this puzzle but no concensus has emerged. Uppal(1992) conducted a survey of the potential explanations and divides them into three main categories.

(1). Home bias is a result of the investors desire to hedge domestic inflation. This potential explanation received a great deal of attention in the early 1980's. Sercu(1980), Adler and Dumas(1983) and Branson and Henderson(1985) all developed models where the desire to hedge inflation resulted from deviations in the law of one price in consumption goods. ${ }^{2}$

\footnotetext{
${ }^{1}$ see French and Poterba(1991), Cooper and Kaplanis(1994) and Tesar and Werner(1995)

${ }^{2}$ Eldor, Pines and Schwartz(1988) and Stockman and Dellas(1989) also generated sim-
} 
They found that as the proportion of domestic goods in total consumption increased, or as the degree of risk aversion increased, the demand for home assets also increased. In these models, the usual response to an increase in the relative risk aversion is a greater demand for domestic bonds, particularly the riskless asset. However, a common feature of these models was that the processes governing prices, the exchange rate and the riskless rate of interest were all specified exogenously. Uppal(1993) discovered that when the process for the exchange rate and the riskless rate of interest were endogenised, this potential explanation of the home bias puzzle was no longer valid. In particular, he found that the model only predicted a bias towards the assets of the home country if the investor had a relative risk aversion less than one. Given that virtually all asset pricing models find a degree of relative risk aversion greater than one ${ }^{3}$, the findings of Uppal(1993) suggest that this hedging motive is unlikely to be the main determinant of the puzzle. Furthermore, Uppal shows that when the degree of relative risk aversion is assigned a more plausible value greater than unity, then the domestic investor actually prefers the foreign stock due to the fact that the exchange rate is negatively correlated with the return on the foreign stock and secondly, the share of the foreign good in total domestic consumption

ilar results by assuming that it was price uncertainty in nontraded goods that prompted investors to hedge against domestic inflation.

${ }^{3}$ see Singleton(1991). 
increases with risk aversion. Therefore the return on the foreign asset is less risky in real terms than the home asset as the investor becomes more risk averse. These findings are supported by Cooper and Kaplanis(1994) who also report that the inflation hedging motive is only valid when the relative risk aversion is very low (less than one).

(2). Home bias occurs due to Institutional Constraints on Foreign Investment. This explanation focuses on direct controls on foreign investment which may restrict inward investment or alternatively restrict domestic investors from investing in foreign securities. Bonser-Neal, Brauer, Neal and Wheatley(1990) tested if such restrictions were binding and found evidence that the French, Japenese, Korean and Mexican markets were partially segmented from the US market. Also Hietala(1989) found evidence that these restrictions may have been a source of bias in the Finnish market. These constraints may be important in certain countries but with financial markets becoming increasingly intergrated this explanation seems unlikely or otherwise we should observe an increasing rate of foreign investment. Halliday(1989) reports that there are no constraints on investing in foreign stock markets in most developed countries. Gultekin, Gultekin and Penati(1989) found evidence of market segmentation between the US and Japanese stock markets prior to the Foreign Exchange and Foreign Trade Control law in 1980 but found no significant evidence of this segmentation in the aftermath 
of this agreement. Furthermore, Fairlamb(1989) investigated if constraints on domestic investors were binding but found that most often, they were not, e.g. in 1988 only $8 \%$ of Spanish funds were invested in foreign assets even though the law allowed for $30 \%$.

(3). Home bias is caused by Discriminatory Taxes and Transaction Costs. Many studies analyse the effects on portfolio choice of discriminatory taxes such as withholding taxes, stamp duties and turnover taxes and transaction costs. A consensus seems to have developed in this area with a number of studies such as, Cooper and Kaplanis(1994), French and Poterba(1991), Kemp(1987), Uppal(1993), Tesar and Werner(1995), agreeing that the level of taxes and transaction costs required to explain such a degree of home bias is much higher than those observed in practice. In particular, Tesar and Werner observe that while international diversification is small, the volume of transactions in international markets is large and increasing. In contrast to what proponents of the high transaction costs detering international diversification argument might believe, Tesar and Werner note that the turnover rate on financial securities held by nonresidents is higher than those held by domestic investors.

Asymmetric information between domestic and foreign investors has been suggested as another possible explanation. For example, domestic investors may have cheaper and more easy access to information about do- 
mestic assets than foreign assets, or alternatively, domestic investors may process the same information differently due to cognitive biases. In an interesting study of the Mexican crisis of 1994, Frankel and Schmukler (1996) conclude that domestic investors may have had better information, and consequently formed more accurate expectations, about local economic events immediately before the crisis than foreign market participants. Frankel and Schmukler find evidence of heterogeneous expectations and show how, just before the devaluation, the Mexican fund Net Asset Values (NAV) (driven by local investors) suffered much faster price falls than Mexican country fund prices (driven by foreign investors). It was found that Mexican NAV's tended to Granger-cause the country fund prices, suggesting that causality flowed from local market participants to Wall Street investors. As a result, local investors were the 'front-runners' in selling Mexican assets in December 1994, and not 'fickle foreign investors' as had been suggested at the time. This provides some, albeit limited, evidence to support the suggestion that asymmetric information may be a significant determinant of home country bias.

Merton (1987) argues that investors are most likely to purchase securities that they are familiar with. This argument is supported by Kang and Stulz (1996) who note that inward foreign investment in Japanese stocks is primarily concentrated in the large domestic companies which have a higher 
international profile. This argument receives support from Tversky and Heath (1991) who present evidence that households perceive an unfamiliar gamble to have greater risk than a familiar one, even when both gambles have identical probability distributions. French and Poterba (1991) argue that home bias could result from investors' feeling safer with, and more optimistic about the prospects of, domestic securities than foreign investors. Schiller, Kon-ya and Tsutsui (1990) present survey evidence consistent with investors often being more optimistic about the domestic market than foreign markets.

Baxter and Jermann(1997) attempt to explain the puzzle by introducing human capital as another asset in the portfolio choice but discover that the puzzle is exacerbated by this extension. This study suggests that the optimal portfolio involves a substantial short position in domestic assets combined with a long position in foreign securities.

In a recent paper, Griffin(1997) attempts to explain some of the observed home bias by showing that pension funds and insurance portfolios (who together dominate the financial markets) will rationally hold a large proportion of domestic assets when the risk associated with asset allocation is measured relative to liabilities. However, this result depends on the length of the liability, with longer liabilities leading to a higher domestic concentration. This is therefore an unlikely explanation of any home bias 
found in this study as we are dealing with monthly portfolio revisions.

Other explanations that have been put forward claim that the problem is not so acute with many domestic investors holding stocks in large multinational companies (Agmon and Lessard(1977)) and with many more large stocks being cross-listed in two or more exchanges. Another simple argument is that we have simply overestimated the benefits of acquiring foreign assets e.g. Bailey and $\operatorname{Stulz}(1990)$. However, despite the wealth of literature that has been afforded to the home bias puzzle, there is still no convincing explanation. Only, the asymmetric information argument remains as a potential solution, yet it is difficult to imagine that this argument is capable of resolving such a deep puzzle.

\subsection{Econometric Methodology and Data}

\subsubsection{The Model}

The aim of this chapter is to identify the optimal portfolio of risky assets available to both UK and US investors. To achieve this we require a model capable of estimating time-varying second moments. We employ a multivariate GARCH model, first presented in chapter 3 and applied in chapter 4 . Consistent with the analysis of the previous chapter, we include a dummy variable for the October ' 87 stock market crash as an extra right 
hand side variable in the conditional mean equation for UK and US equities. Therefore the model which we estimate is as follows:

$$
\begin{aligned}
\mathbf{r}_{t} & =\boldsymbol{\alpha}+\boldsymbol{\beta} \mathbf{r}_{t-1}+\boldsymbol{\gamma d u m} 87+\boldsymbol{\xi}_{t} \\
\boldsymbol{\xi}_{t} & \mid \Psi_{t-1} \sim N\left(0, \mathbf{H}_{t}\right) \\
\mathbf{H}_{t} & =\mathbf{V}^{\prime} \mathbf{V}+\mathbf{A}^{\prime}\left(\mathbf{H}_{t-1}-\mathbf{V}^{\prime} \mathbf{V}\right) \mathbf{A}+\mathbf{B}^{\prime}\left(\boldsymbol{\xi}_{t-1} \boldsymbol{\xi}_{t-1}^{\prime}-\mathbf{V}^{\prime} \mathbf{V}\right) \mathbf{B}
\end{aligned}
$$

where $\mathbf{r}=(u k e q, u s e q, g v b d)^{\prime}$ and $u k e q, u s e q$ and $g v b d$ refer to UK equities, US equities and a domestic government bond. $\boldsymbol{\beta}$ is a $3 x 3$ matrix of regression parameters and $\boldsymbol{\gamma}$ is a $3 x 1$ vector of parameters. Once more, this parsimonious representation ensures that the resulting matrices are positive definite while allowing the user to disentangle long- and short-run effects in the conditional second order moments.

\subsubsection{The Data}

This chapter uses time series data on broad classes of UK and US financial assets. The analysis is conducted, firstly from the perspective of a UK investor and secondly with respect to a US investor. In each case, we focus on four assets, three risky assets and a riskless one. Both sets of investors have the opportunity to invest in two domestic risky assets, a domestic risk- 
less asset and one foreign risky asset. The risky assets used in the analysis are UK equities, represented by the Financial Times All Share Index; US Equities represented by the S\&P Composite Index, UK government bonds represented by the FT British government stock index; US bonds represented by an all government bond index. In each case, the return on the foreign asset is converted into the domestic currency using end of month exchange rates. The data used in this chapter is annualised monthly total returns for each asset and is calculated so as to take account of dividend payments in the case of equities and coupon payments in the case of government bonds. For the UK investor, the rate of return on the UK government 30 day Treasury bill is taken as the risk free rate of interest while for the US investor, the riskless interest rate is proxied by the Eurodollar rate, i.e. the rate available on one month US deposits in London. It is true to say that these assets are riskless at least in the nominal sense. All data was sourced from DATASTREAM.

The data covers a sample period beginning in January, 1980 and finishing in September, 1996. Again we have chosen to work exclusively with rates of return in excess of the risk free rate in order to avoid over-estimating the total risk of the portfolio. As noted previously, this also avoids unit root problems in the data since a unit root is rejected for all of the excess returns. 


\subsection{Estimation Results}

The model as specified in equation (5.1) was maximised subject to the Berndt, Hall, Hall and Hausmann (BHHH) algorithm. The results of the model for both the UK and US investor are reported below with $t$-statistics in parentheses.

\subsubsection{UK Investor}

\section{Mean Matrices}

$$
\alpha=\left[\begin{array}{c}
7.23 \\
(2.2) \\
5.20 \\
(1.23) \\
-0.61 \\
(-0.33)
\end{array}\right], \boldsymbol{\beta}=\left[\begin{array}{ccc}
-0.06 & 0.07 & 0.20 \\
(-0.74) & (1.07) & (1.32) \\
-0.09 & 0.11 & 0.07 \\
(-1.05) & (1.39) & (0.43) \\
0.01 & -0.06 & 0.10 \\
(0.27) & (-1.95) & (1.35)
\end{array}\right], \gamma=\left[\begin{array}{c}
-403.51 \\
(-1.49) \\
-334.39 \\
(-2.01) \\
0
\end{array}\right]
$$




\section{Covariance Matrices}

$$
\mathbf{V}=\left[\begin{array}{ccc}
57.21 & & \\
(8.99) & & \\
26.59 & 56.98 & \\
(4.51) & (11.94) & \\
15.50 & -4.94 & 24.06 \\
(7.17) & (-2.17) & (11.4)
\end{array}\right]
$$

$$
\mathbf{B}=\left[\begin{array}{ccc}
0.27 & & \\
(3.10) & & \\
-0.13 & 0.28 & \\
(-2.43) & (3.86) & \\
0.15 & 0.02 & 0.43 \\
(4.28) & (0.51) & (4.08)
\end{array}\right], \mathbf{A}=\left[\begin{array}{cccc}
-0.80 & & \\
(-7.45) & & \\
-0.19 & -0.73 & \\
(-2.01) & (-5.5) & \\
-0.15 & -0.01 & 0.39 \\
(-0.97) & (-0.07) & (3.05)
\end{array}\right]
$$

\section{Discussion of the Results}

In the conditional mean the elements of $\boldsymbol{\beta}$ are generally not significant. This is consistent with the usual finding that total stock and bond returns are serially uncorrelated. The most significant element is $\beta_{32}$ implying that the lagged excess return on the US equity has some explanatory power for the 
excess return on UK bonds, but it is difficult to think of a good reason why this should be.

In contrast, the conditional second order moments are much more readily explained by the GARCH structure adopted in the model. All the elements of the $\mathbf{V}$ matrix, which determines the unconditional or long-run matrix, are statistically significant. This observation is supportive of the hypothesis that financial data exhibit volatility clustering even at monthly horizons. Furthermore, the statistical significance of the off-diagonal elements of the long-run matrix suggests that there are volatility spillovers from market to market. Also, the short-run matrices, $\mathbf{A}$ and $\mathbf{B}$, are characterised by many statistically significant parameters. The significance of the diagonal elements of $\mathbf{A}$ and $\mathbf{B}$ indicates that the conditional variances differ considerably from the unconditional variances. The results show that not alone are own lagged volatility measures important in determining the current level but again there is evidence of contagion effects between markets, especially between the UK and US stockmarkets with both $A_{21}$ and $B_{21}$ being statistically significant parameters. Short-run volatility in the UK bond market is influenced by the UK equity market, see $B_{31}$ but appears to be segmented from the US stockmarket, evidenced by the non-significance of both $A_{32}$ and $B_{32}$. The significance of $\{3,1\}$ elements suggests that the allocation between UK equity and UK bonds will need to be re-balanced in the short 
run to achieve optimality.

The significance of the $\{2,1\}$ elements of $\mathbf{A}$ and $\mathbf{B}$ together with the $\{2,1\}$ element of the long-run covariance matrix, is the reason why investors may want to hold an internationally diversified portfolio in order to reduce risk. For example, the long-run covariance matrix is

$$
\mathbf{V}^{\prime} \mathbf{V}=\left[\begin{array}{lll}
3273 & & \\
1521 & 3953 & \\
887 & 131 & 843
\end{array}\right]
$$

implying a correlation between the excess returns over the UK risk-free rate of UK and US equity returns of 0.42 . They also imply that to achieve an optimal portfolio there will need to be a re-balancing between UK and US equity. 
5.4.2 Ů $\mathrm{S}_{1} \mathrm{v}$ stor

Méan hatatri es

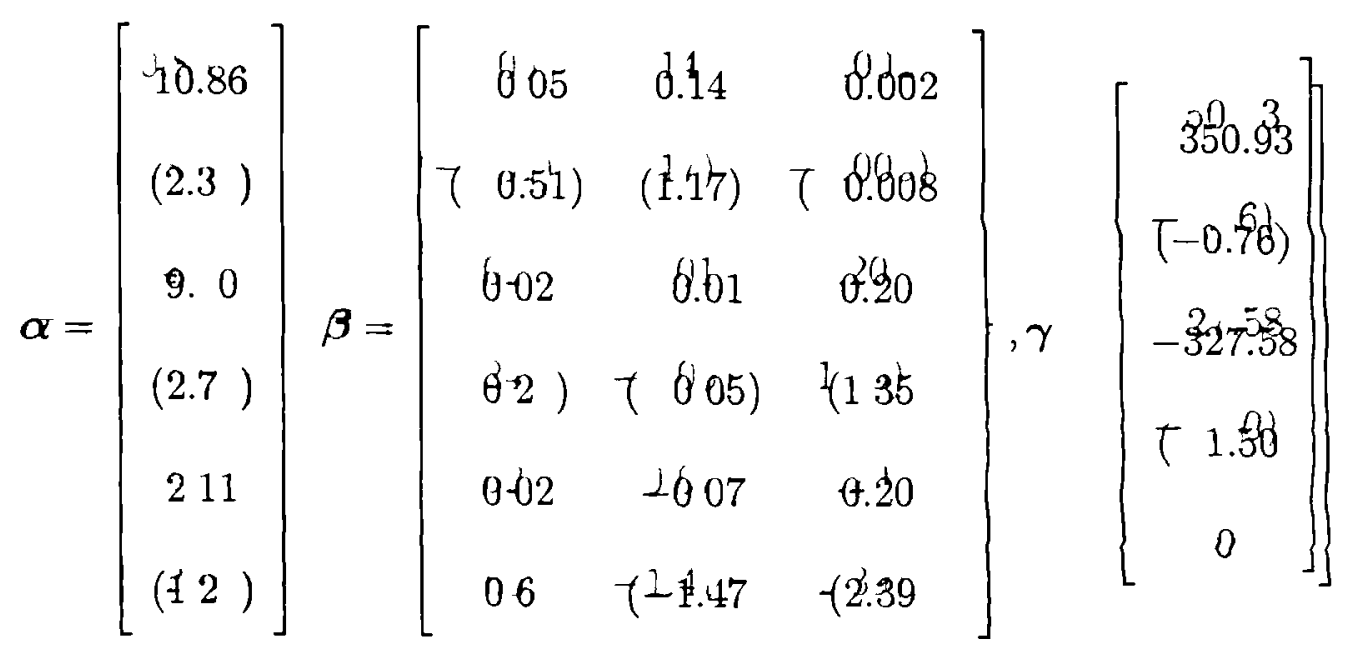

Covariance Matı iccs

$$
V=\left[\begin{array}{ccc}
646 & & \\
(1503 & & \\
22.4 & 3999 \\
(5.96) & 1617) & \\
5.50 & 6.5 & 185 \\
3.26 & (3.91) & -(194
\end{array}\right]
$$




$$
\mathbf{A}=\left[\begin{array}{ccc}
-0.38 & & \\
(-0.95) & & \\
-0.05 & 0.24 & \\
(-0.15) & (0.60) & \\
0.39 & -0.41 & 0.47 \\
(1.61) & (-1.99) & (2.10)
\end{array}\right], \mathbf{B}=\left[\begin{array}{ccc}
-0.32 & & \\
(-1.78) & & \\
0.10 & -0.26 & \\
(0.09) & (-2.08) & \\
-0.02 & -0.21 & 0.22 \\
(-0.35) & (-3.46) & (2.65)
\end{array}\right]
$$

The results are similar to those for the UK. Again, $\boldsymbol{\beta}$ is almost insignificant, though here there does seem to be some significant persistence in the excess return on US bonds. As in the case of the UK, the $\operatorname{GARCH}(1,1)$ structure is very successful in explaining the conditional volatility. All the elements of the long-run unconditional matrix, $\mathrm{V}$, are statistically significant. Once more this is supportive of volatility spillovers between the markets in the long run. Volatility is transmitted between international markets and shows that there is a high level of intergration between the UK and US financial markets. Also the $\mathbf{A}$ and $\mathbf{B}$ matrices are strongly supportive of important variances and covariances in the short run, especially the covariance between the two US assets. There is strong support for contagion effects between US stock and bond markets with both $A_{32}$ and $B_{32}$ having $t$-statistics greater than 1.96 . The significance of these elements 
indicates that the optimal asset allocation can only be attained by short-run re-balancing between US equity and US bonds. Once more the domestic bond market appears to be segmented from volatility spillovers originating in the foreign equity market with the parameters linking UK equity and US bonds being indistinguishable from zero.

The main difference is that there are no significant contagion effects between the US and UK stockmarkets. Taken together, the UK and US results seem to indicate that causality runs from the US to the UK stockmarket. It would also suggest that the gains to the US investor from re-balancing the portfolio in the short run between US and UK assets are likely to be small. This is not to suggest that there aren't likely to be gains to the US investor to holding UK equity. The long-run covariance matrix is

$$
\mathbf{V}^{\prime} \mathbf{V}=\left[\begin{array}{ccc}
4173 & & \\
1453 & 2034 & \\
355 & 389 & 419
\end{array}\right]
$$

giving a correlation between the excess returns over the US risk-free rate on US and UK equity of 0.50 . 


\subsection{Optimal Asset Allocation}

\subsubsection{Frontier Movements}

Figures 5-1 and 5-2 show the conditional variances for each of the risky assets denominated in UK pounds and US dollars respectively. These graphs show the long-run value of the variance and also the total variance containing the short-run deviations. It is clear that short-run deviations can be quite large, and are therefore likely to have a significant impact on the portfolio frontiers and hence on asset allocation in the short run. For each country fluctuations in the exchange rate make foreign equity the asset with the most volatile excess returns. Nonetheless, since 1993, there has been a noticeable decline in volatility for all assets, and especially for equity returns expressed in sterling. This reflects the relative stability of the $£ / \$$ exchange rate over this period.

Next we generate the portfolio frontiers for both sets of investors. The position of the frontiers reflect the minimum portfolio standard deviation for a given portfolio return, hence this is just another way of comparing portfolio standard deviations. Interestingly, we find that the US investor enjoys a 'risk-return' advantage over his UK counterpart. Figures 5-3 and 5-4 show this. In figure 5-3, we plot the mean frontier for each investor and see that the US frontier lies inside the UK frontier at all points. This means 
that the US investor has to bear less risk than a UK investor to achieve the same return. Figure 5-4 provides more information on the distribution of frontier movements. It displays the maximum, minimum and mean frontiers for each country and we find that each US frontier (light line) is consistently located nearer to the origin than the corresponding UK frontier (thick line). This result would suggest that the optimal portfolio of risky assets available to the US investor should deliver a higher Sharpe Performance Index than the equivalent portfolio in the UK. This graph also shows that although the conditional distribution of frontiers for the US investor is shifted to the left of that for the UK investor, there is considerable overlap in the distributions and it indicates that the conditional distributions are positively skewed, with a few periods when portfolio risk is much higher than the mean.

\subsubsection{Optimal Portfolios}

Once more the aim of the study is not to determine the final investment position but rather to identify the time-varying constituents of the optimal portfolio of risky assets for each investor. The location of the optimal portfolio when there are no restrictions on short sales is obtained from the point of tangency between the portfolio frontier and the Capital Market line which goes through the origin. Figures 5-5 and 5-6 show the behaviour of both the excess return and the standard deviation for the UK and US optimal 
portfolios respectively. Consistent with the location of the frontiers, we find that the US portfolio delivers a higher mean excess return, $9.1 \%$ versus $8 \%$. Figure 5-7 shows the Sharpe Performance Index $\left(S P I_{t}=\frac{r e t u r n_{t}}{r i s k_{t}}\right)$ for each portfolio. On average, the US portfolio achieves a higher SPI, 0.24 versus 0.18 and is much more stable than for the UK portfolio. However, the most noteworthy feature of the figure is the remarkable improvement in the UK SPI since 1992 due to a combination of the strong and persistent growth of equity prices, and the relative tranquility of the stockmarkets, see figures 5-1 and 5-2. As a result the SPI for the UK has exceeded that for the US since the end of 1994.

\section{UK Investor}

Next we seek to identify the proportions in which each asset must be held in order for the investor to hold the optimal portfolio of risky assets. Initially, the allocation of funds for investment in risky assets was unrestricted, allowing the investor to take unlimited short positions. Firstly, looking at the UK investor, figure 5-8 shows the relative importance of each asset class over the entire sample. We see that the UK equity dominates the portfolio, accounting on average for $77 \%$ of the investment. However, the asset holding fluctuates a great deal but is never held short. In fact, in many cases more than $100 \%$ of the investor's wealth is held in the domestic eq- 
uity. On the other hand, the UK bond is often held short to allow the extra investment in equity. On average, the UK bond accounts for $-4 \%$ of the investment. The investment in bonds is very volatile and varies between a maximum of $55 \%$ to a minimum of $-166 \%$. Throughout the sample period, the investment in US equity is relatively stable and has a mean holding of $27 \%$. It is held short in only one period during the entire 200 period sample. It is also clear from figure $5-8$ that the improved SPI is due entirely to the increased investment in domestic equity with a corresponding decline in the holding of the domestic bond. Obviously the combination of rising return and declining conditional volatility increased the attractiveness of domestic equity in the post-1993 sample. Table 5.1 contains a summary of the unrestricted allocation over the entire sample.

\begin{tabular}{|l|c|c|c|}
\hline & Mean & Minimum & Maximum \\
\hline UK Equity & $77 \%$ & $24 \%$ & $210 \%$ \\
\hline US Equity & $27 \%$ & $-2 \%$ & $60 \%$ \\
\hline UK Bond & $-4 \%$ & $-166 \%$ & $55 \%$ \\
\hline
\end{tabular}

Table 5.1: Summary statistics for optimal unrestricted UK portfolio

Of course such an investment strategy may be deemed too costly and time consuming to implement as the frequent revisions in the position of the individual assets are likely to incur transaction costs although the use of indexed trackers or futures would help make it more feasible. A more 
realistic strategy would be to form portfolios that prohibit short sales. This is a worthwhile exercise since UK mutual fund managers are restricted by law to holding only non-negative quantities of an asset. These portfolios are constructed to deliver the average excess return on the unrestricted portfolio.

Figure 5-9 shows how the constituents of the optimal portfolio have varied over the sample. UK equity still dominates the portfolio with a mean of $71 \%$, but its range of variation is reduced by a factor of about 9 , having a maximum of $89 \%$ and a minimum of $62 \%$. The mean share of US equity is similar and its range of variation is halved. The mean share of UK bonds is $4 \%$, and its range of variation is reduced by a factor of about 20 . Now that borrowing by selling domestic bonds is prohibited, portfolio re-balancing takes place mainly between domestic and foreign equity. This results in a considerable reduction in the degree of re-balancing. Table 5.2 contains the summary of the restricted holdings of each asset over the period.

\begin{tabular}{|l|c|c|c|}
\hline & Mean & Minimum & Maximum \\
\hline UK Equity & $71 \%$ & $62 \%$ & $89 \%$ \\
\hline US Equity & $25 \%$ & $0 \%$ & $38 \%$ \\
\hline UK Bond & $4 \%$ & $0 \%$ & $11 \%$ \\
\hline
\end{tabular}

Table 5.2: Summary statistics for UK restricted portfolio 
Survey evidence shows that UK investors hold up to $18 \%$ of their wealth in foreign assets. Our estimate is that a UK investor faced with the opportunity to form an optimal portfolio from these three risky assets should hold about $25 \%$ of wealth in US equity. The difference between the two is a measure of the extent of home bias by UK investors.

\section{US Investor}

A similar analysis was conducted for the US investor. Figure 5-10 shows how the holdings of the individual assets behaved over the sample. Again, the domestic equity dominates the portfolio. On average the investment in domestic equity accounted for $64 \%$ of the funds invested in risky assets. We observe that the holding of this asset fluctuates wildly, moving between a range of $30 \%$ to $332 \%$. Whenever in excess of $100 \%$ of wealth is invested in US equity, it is always funded by adopting a short position in the domestic bond. This asset is consequently very volatile but in the mean has a long investment position of $16 \%$. The UK equity contributes a very substantial amount to the optimal portfolio with a mean position of $20 \%$ and is never held short. Therefore, it makes a positive contribution to the portfolio in each of the 200 months in the sample. Table 5.3 summarises this investment strategy; 


\begin{tabular}{|l|c|c|c|}
\hline & Mean & Minimum & Maximum \\
\hline UK Equity & $20 \%$ & $2 \%$ & $109 \%$ \\
\hline US Equity & $64 \%$ & $30 \%$ & $332 \%$ \\
\hline US Bond & $16 \%$ & $-342 \%$ & $57 \%$ \\
\hline
\end{tabular}

Table 5.3: Summary statistics for US unrestricted portfolio

Again, this investment strategy looks excessively volatile and we restrict the investor so that only non-negative positions can be assumed. Figure 5-11 shows the constituents of this portfolio over time and table 5.4 summarises the restricted investment strategy for the US investor.

\begin{tabular}{|l|c|c|c|}
\hline & Mean & Minimum & Maximum \\
\hline UK Equity & $20 \%$ & $0 \%$ & $43 \%$ \\
\hline US Equity & $63 \%$ & $38 \%$ & $86 \%$ \\
\hline US Bond & $17 \%$ & $14 \%$ & $20 \%$ \\
\hline
\end{tabular}

Table 5.4: Summary statistic for optimal restricted US portfolio

The mean shares are hardly altered but the variation in the shares is greatly reduced compared with the unrestricted portfolio. Again, US equity dominates, having a mean investment position of $63 \%$ and a considerably smaller range of $38 \%$ to $86 \%$. The investment in the domestic bond is remarkably stable moving only between $14 \%$ and $20 \%$ of the portfolio and on average accounting for $17 \%$. As in the UK model, the restricted portfolio gives rise to a negative relationship between the domestic and foreign equity. An increased investment in one is offset with a reduced position in the other, leaving the domestic bond relatively unchanged. Despite this, the 
UK equity again contributes $20 \%$ of the average investment and is very important relative to what observed behaviour might suggest.

Survey evidence shows that US investors hold as little as $6 \%$ of their wealth in foreign assets. This compares with our estimate that $20 \%$ should be allocated to UK equity. The home bias problem therefore seems to be much more a feature of US than UK investment.

\subsection{Conclusion}

The aim of this chapter has been to re-examine the issue of the optimal tactical asset allocation of an internationally diversified portfolio. These results are then used to provide new ways of determining whether the location of an investor ought to affect portfolio selection, and of addressing the home-bias puzzle. The example used in the analysis is the optimal mix of domestic and foreign equity, and domestic bonds that should be held by UK and US investors, two countries that have not had significant barriers to investing abroad for some time. Two tactical investment strategies are compared. Both are versions of Markowitz's mean-variance portfolio theory in which investors use the joint conditional distribution of excess returns, which is time varying, to re-balance their portfolios each period. One allows investors to hold unlimited short positions; the other assumes that investors are constrained from going short, the situation faced by most 
fund managers. The conditional covariance matrix is estimated using a multivariate GARCH model.

We find that for both UK and US investors, although domestic equity is the dominant asset, it is optimal to hold between $20 \%$ and $27 \%$ of wealth in foreign equity. This compares with survey evidence which indicates that in practice UK investors hold around $18 \%$ in foreign assets, while US investors hold only about $6 \%$. The home-bias puzzle seems therefore to be more acute for US than UK investors. Put another way, there seems to be more potential gains from increased international diversification for the US than the UK investor.

We also find that the location of the investor is important in determining the investment performance of the portfolio. The portfolio frontiers facing the US investor lie nearer the origin than for the UK investor, implying that US investors can achieve the same return while bearing less risk. This 'riskreturn' advantage is also shown in the higher average Sharpe Performance Index for the US - even though since 1993 the SPI for the UK has steadily improved and now lies above that for the US.

Furthermore, our results provide evidence of contagion effects across markets. We note that in the long run, regardless of the location of the investor, there are volatility spillovers between all markets. Volatility is transmitted between both domestic and international markets. In the short 
run we find that volatility in the UK equity market is influenced by the US stockmarket but volatility is not transmitted in the opposite direction. Also, stockmarket volatility spills over to the domestic bond market, but in the short run bond markets appear to be segmented from foreign equity markets.

In summary, we can say that the potential gains from international diversification are not being fully exploited by either the UK or the US investor. Hence, the home-bias puzzle still remains. Secondly, the US investor appears to have a 'risk-return' advantage over his UK counterpart, resulting in a better average performance from the US portfolio. 
Figure 5.1

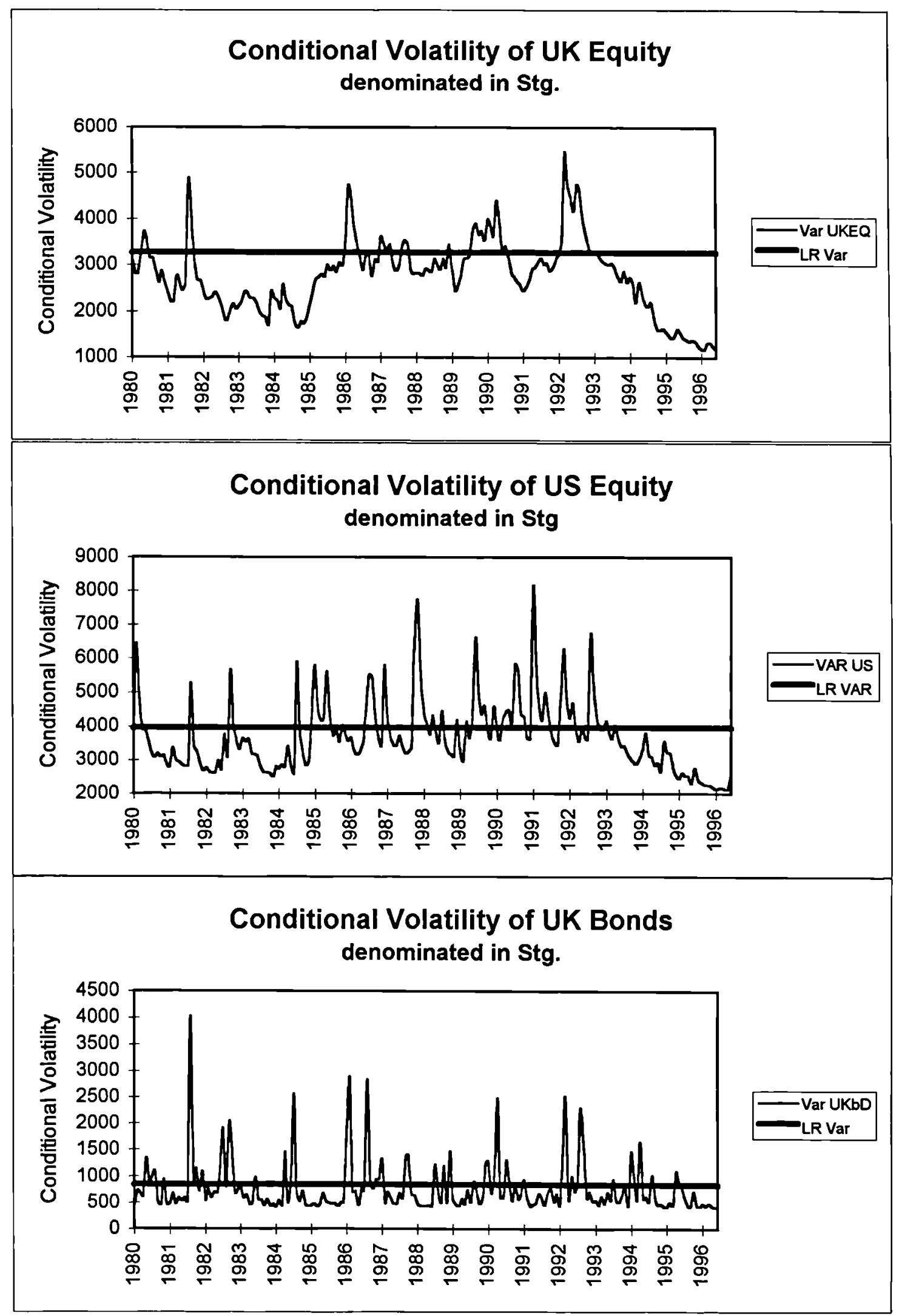


Figure $\mathbf{5 . 2}$

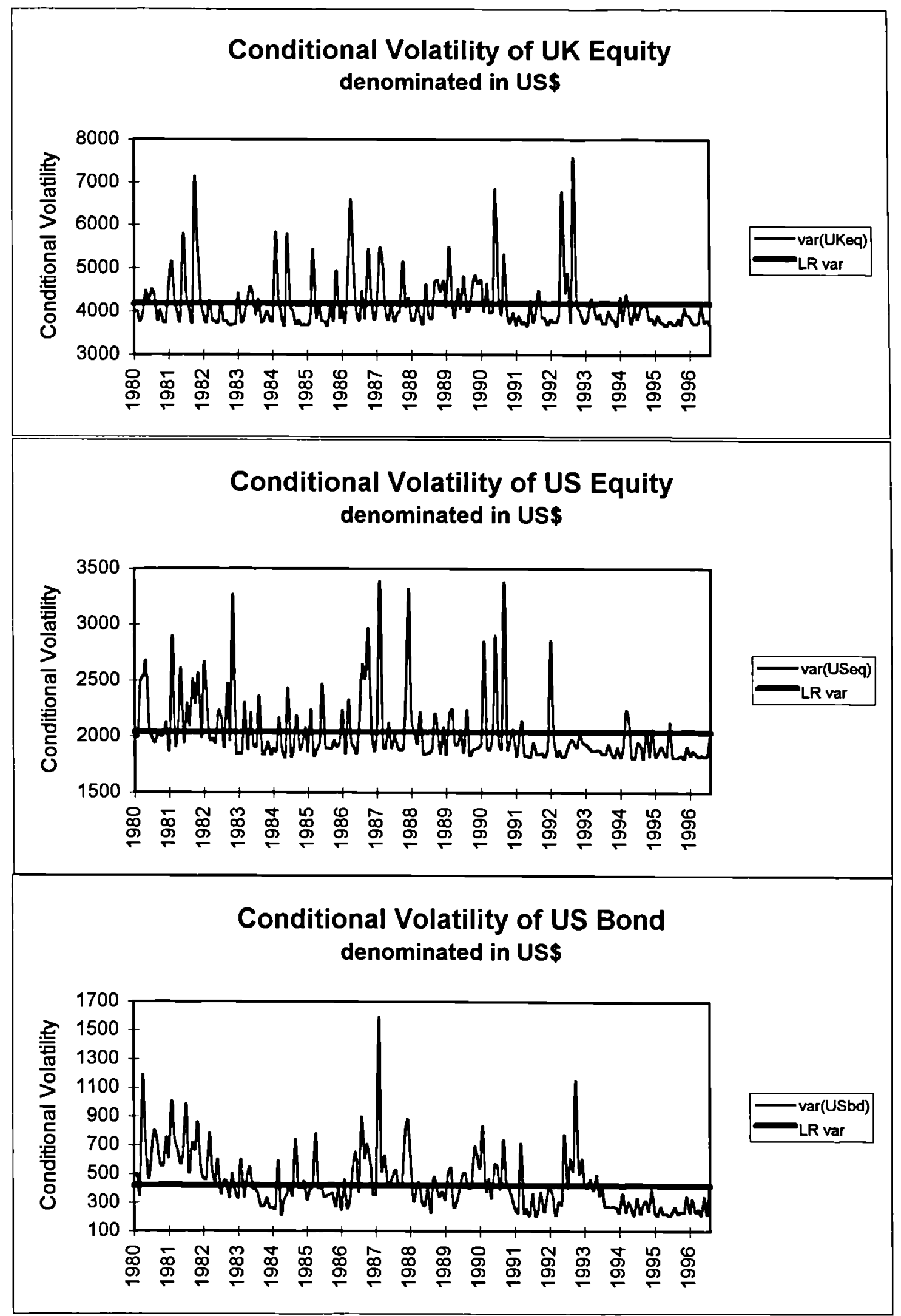




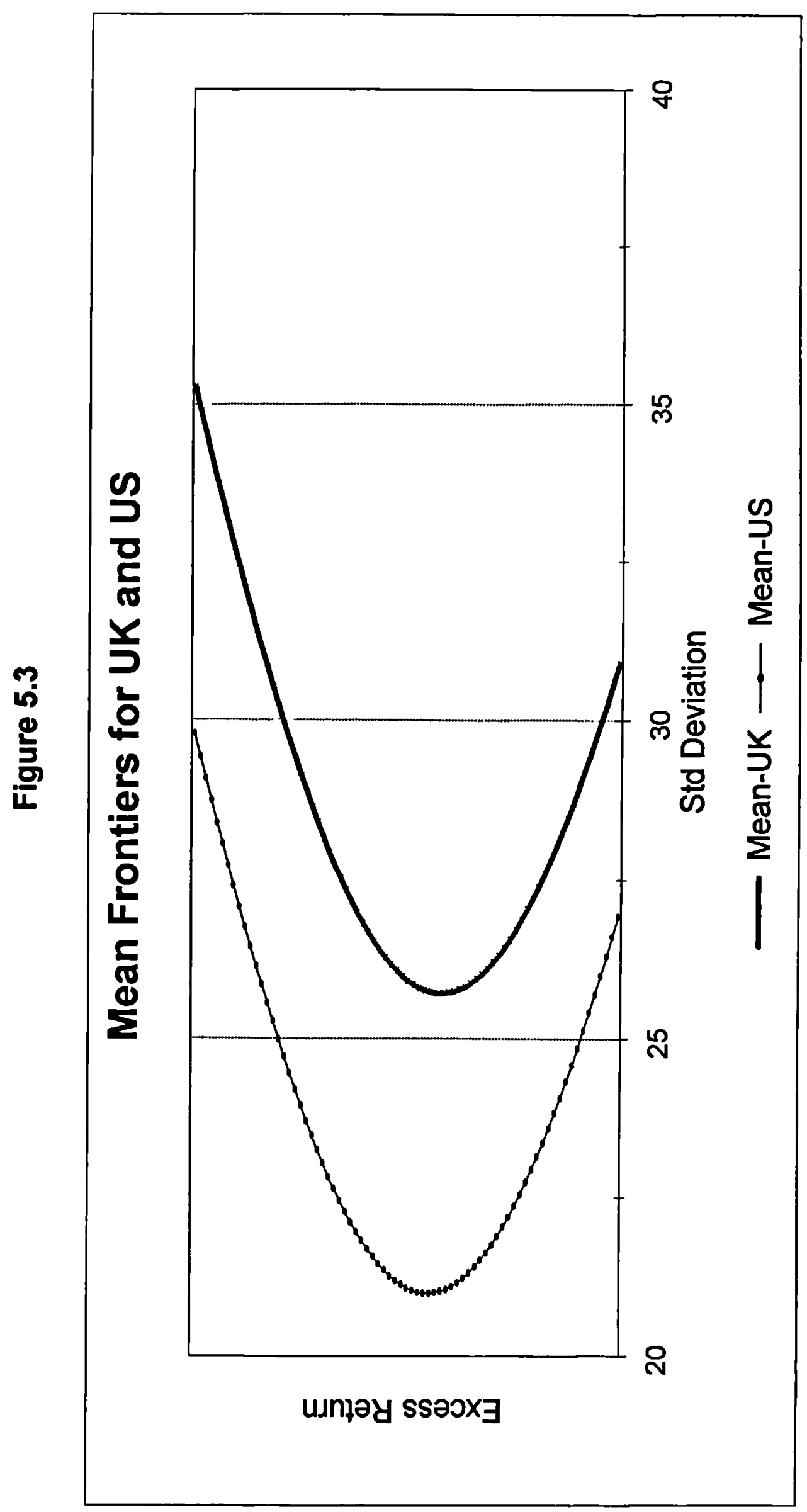

$\stackrel{M}{N}$ 


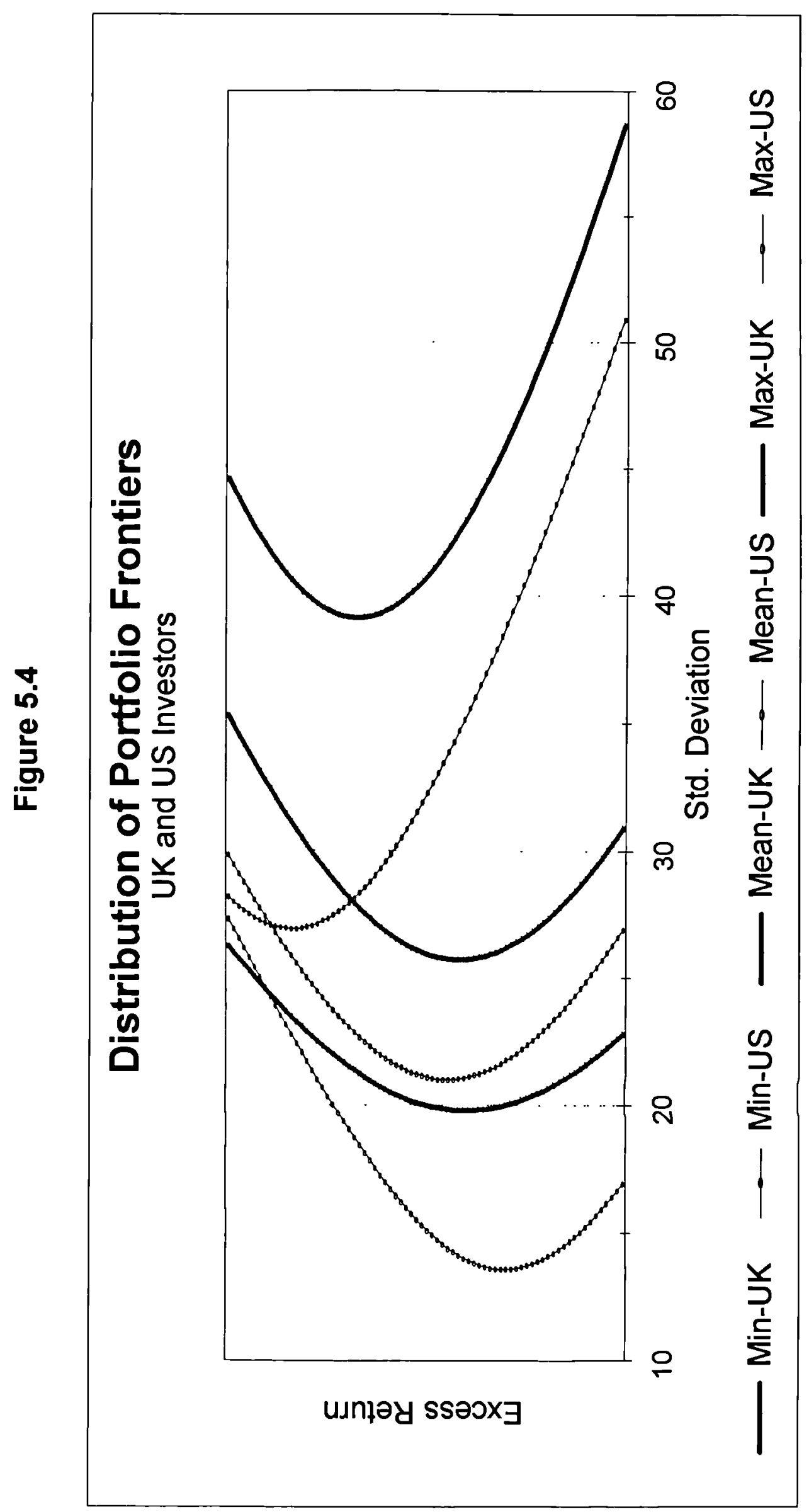

$\stackrel{+}{\sim}$ 
Figure 5.5

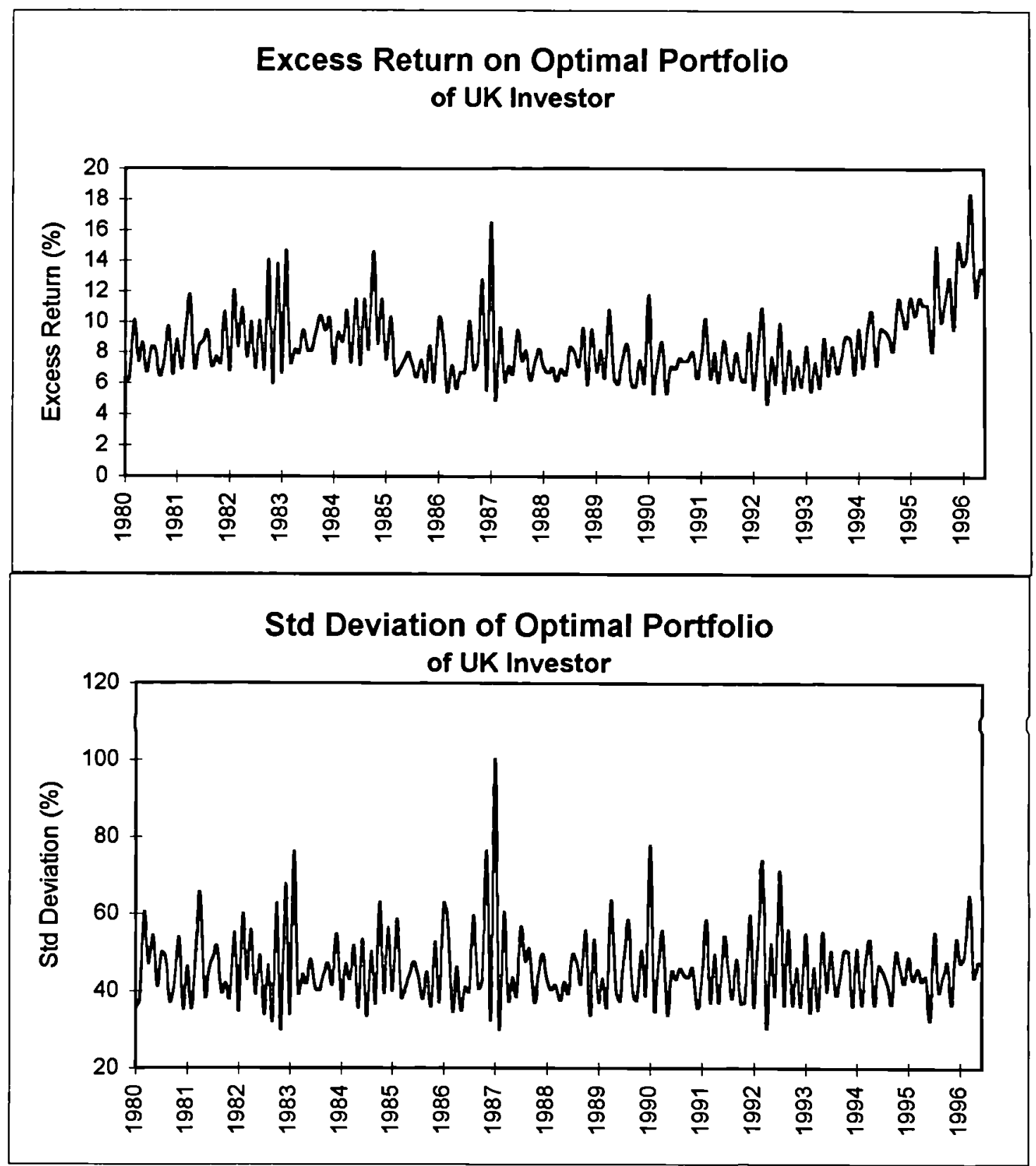


Figure 5.6

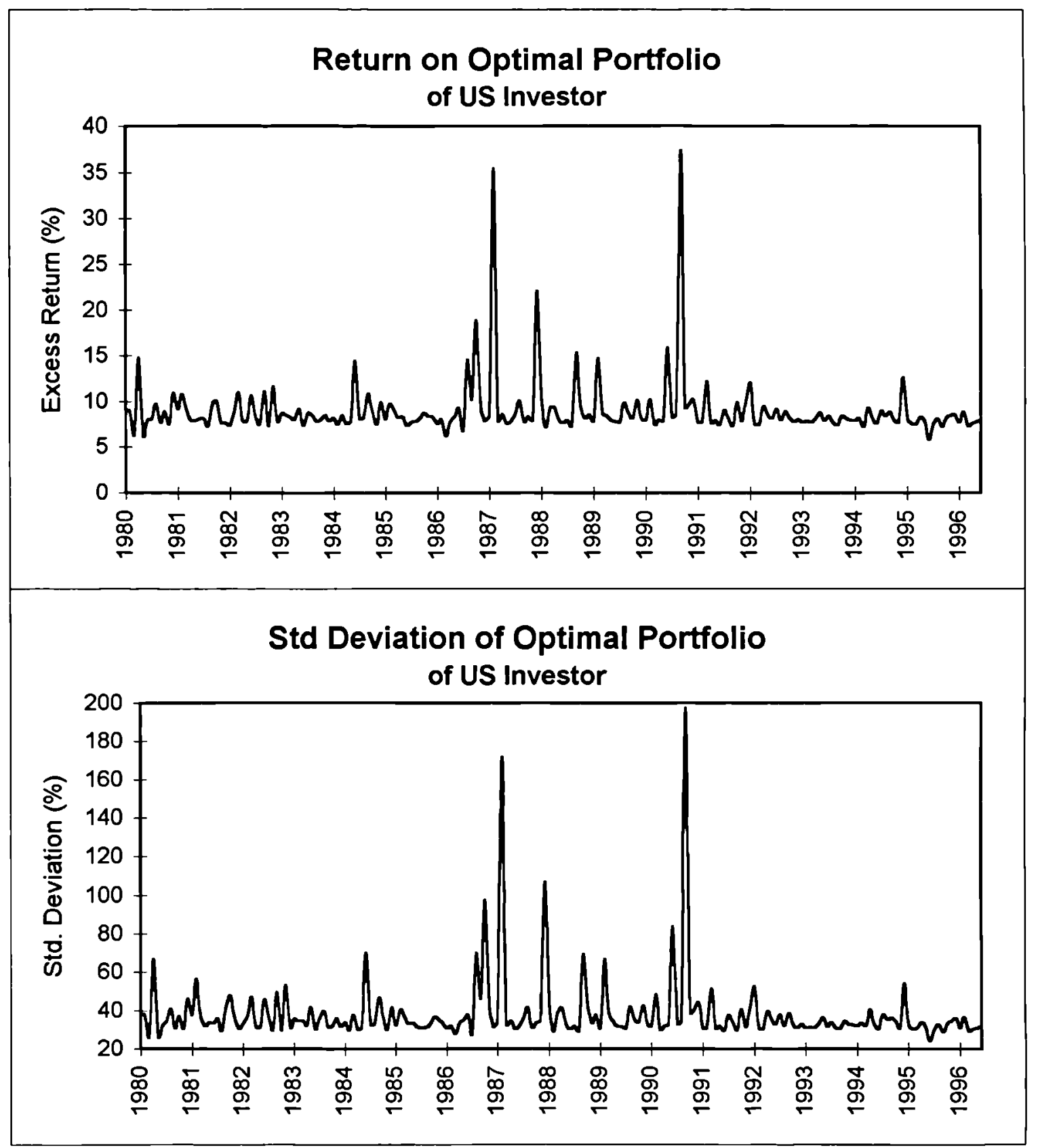



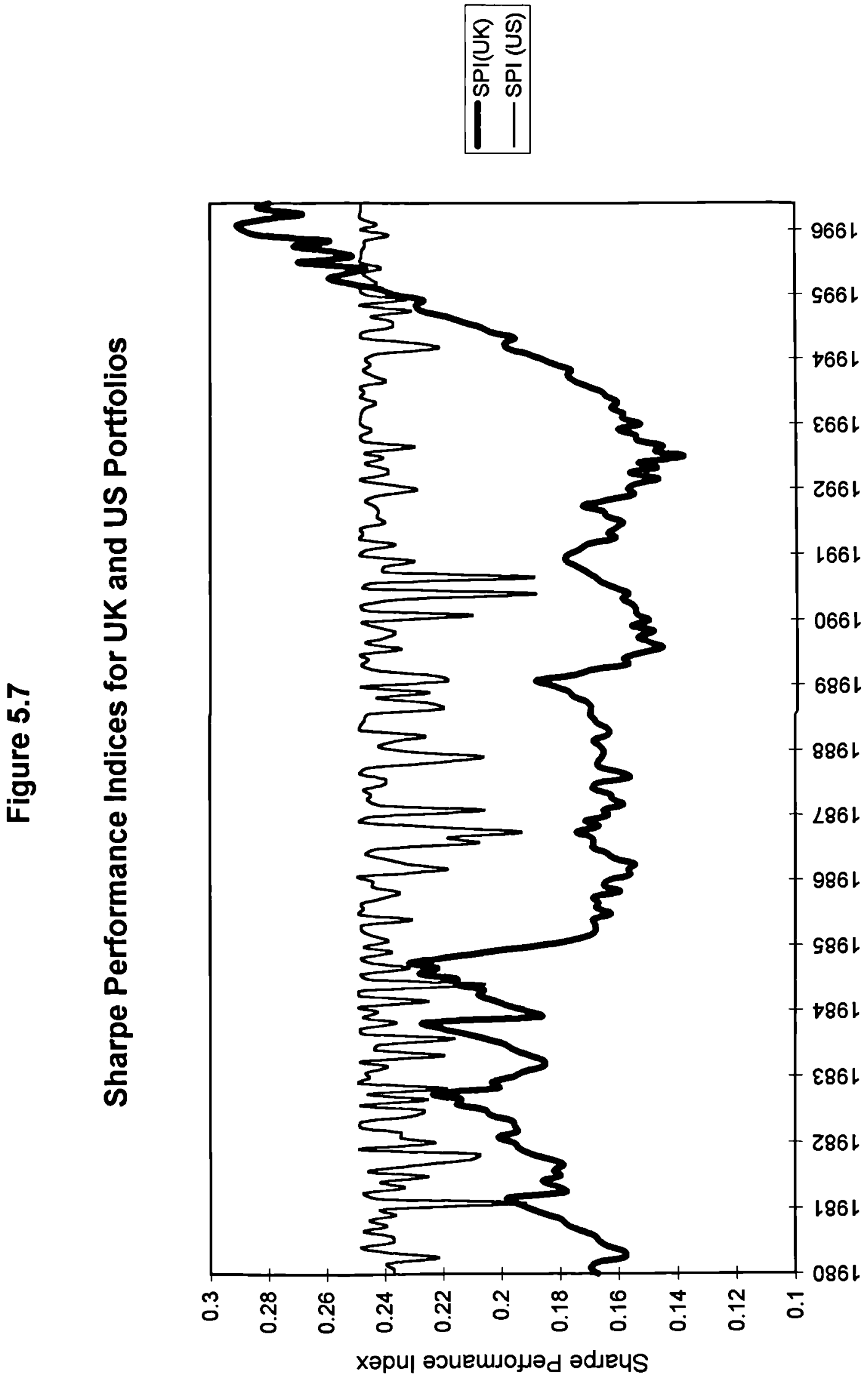


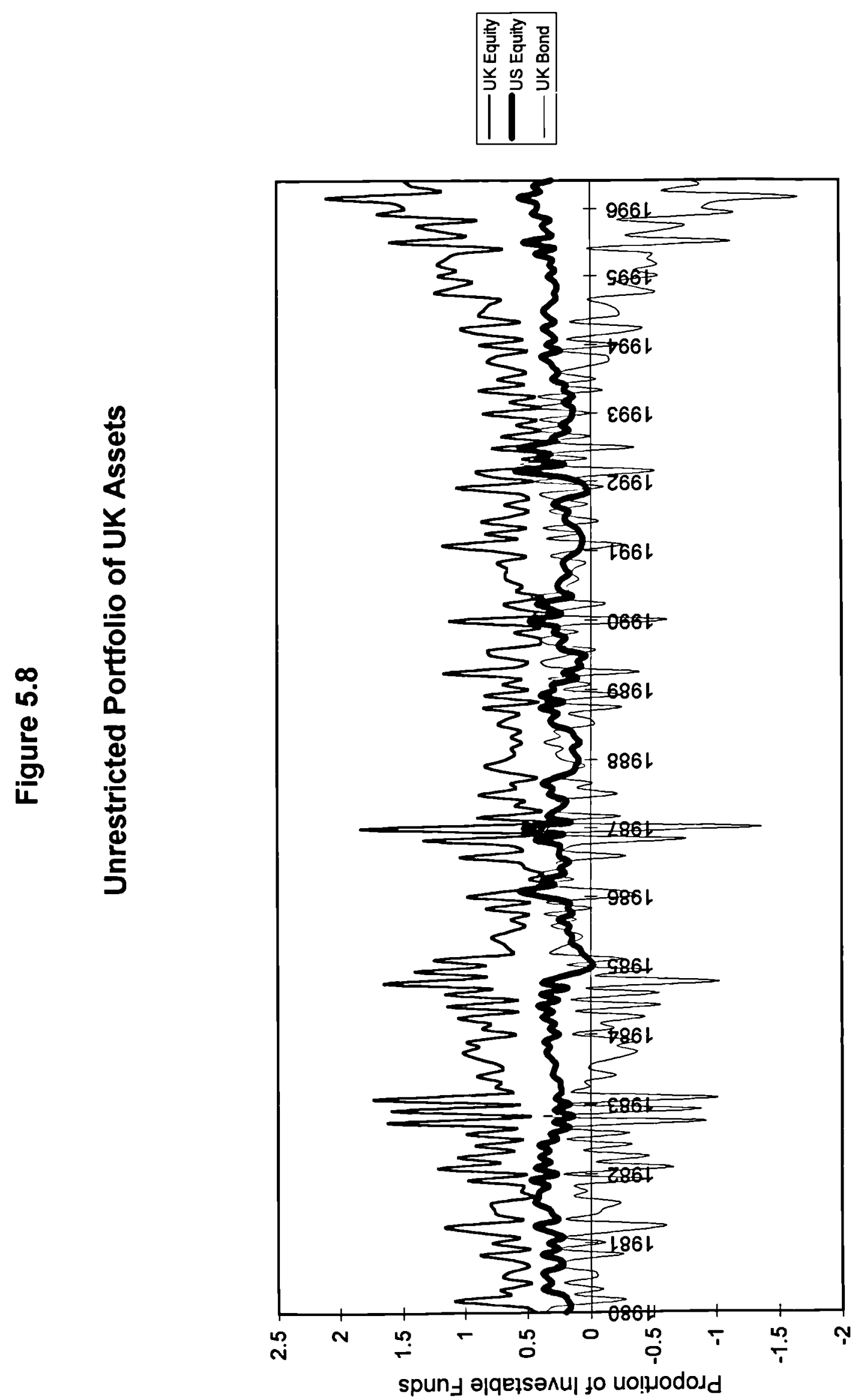

$\stackrel{\infty}{\sim}$ 


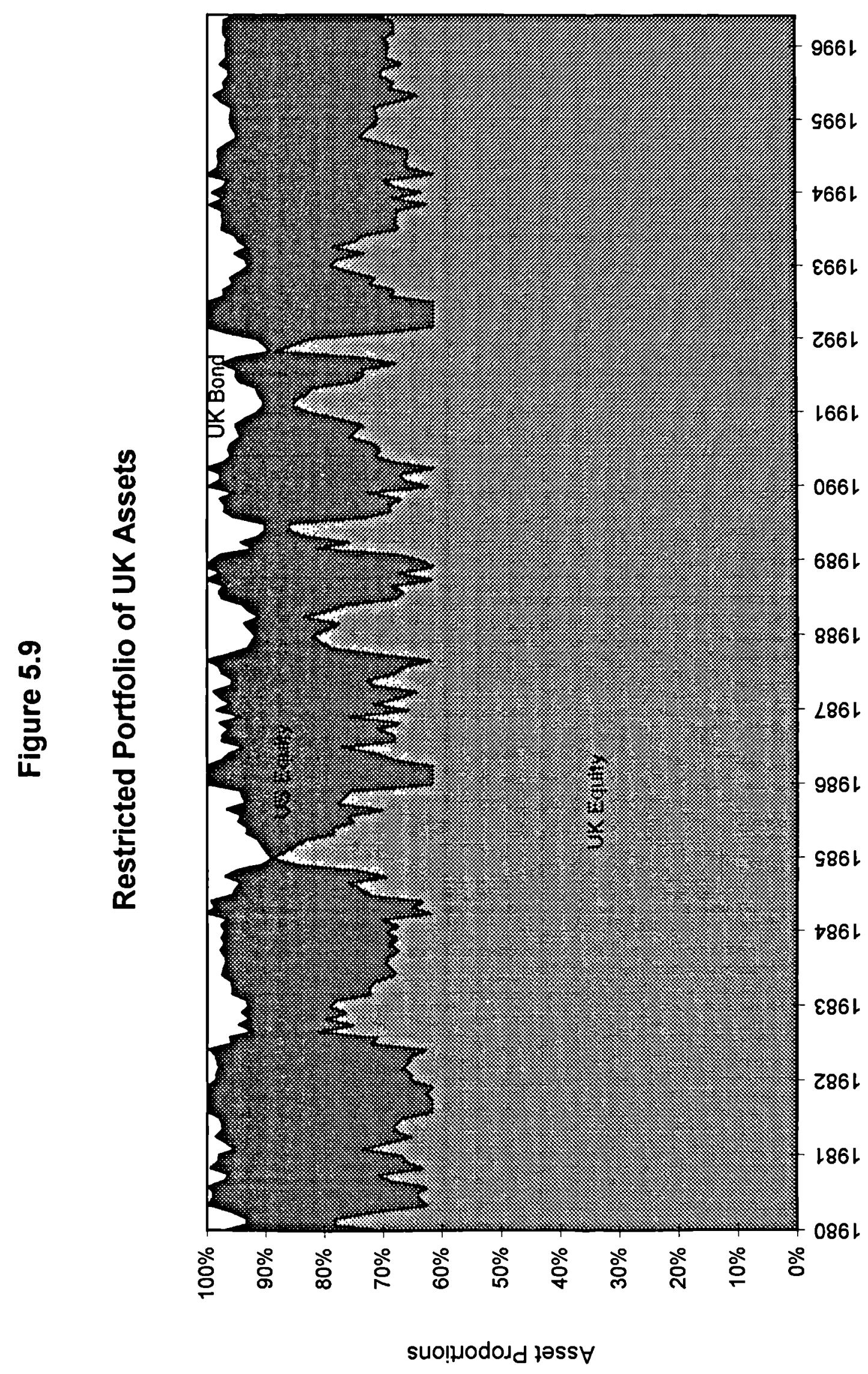



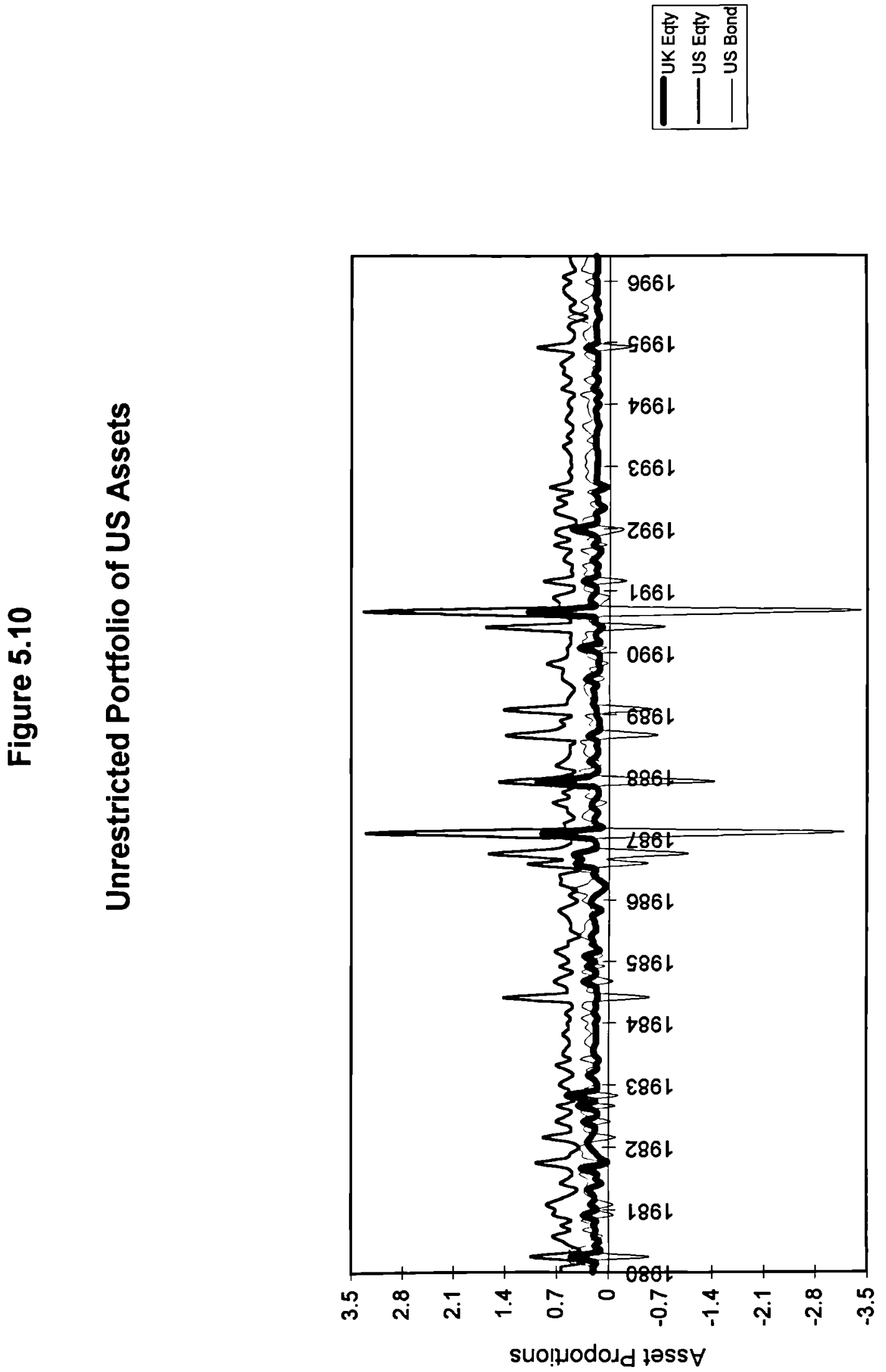

ำ 


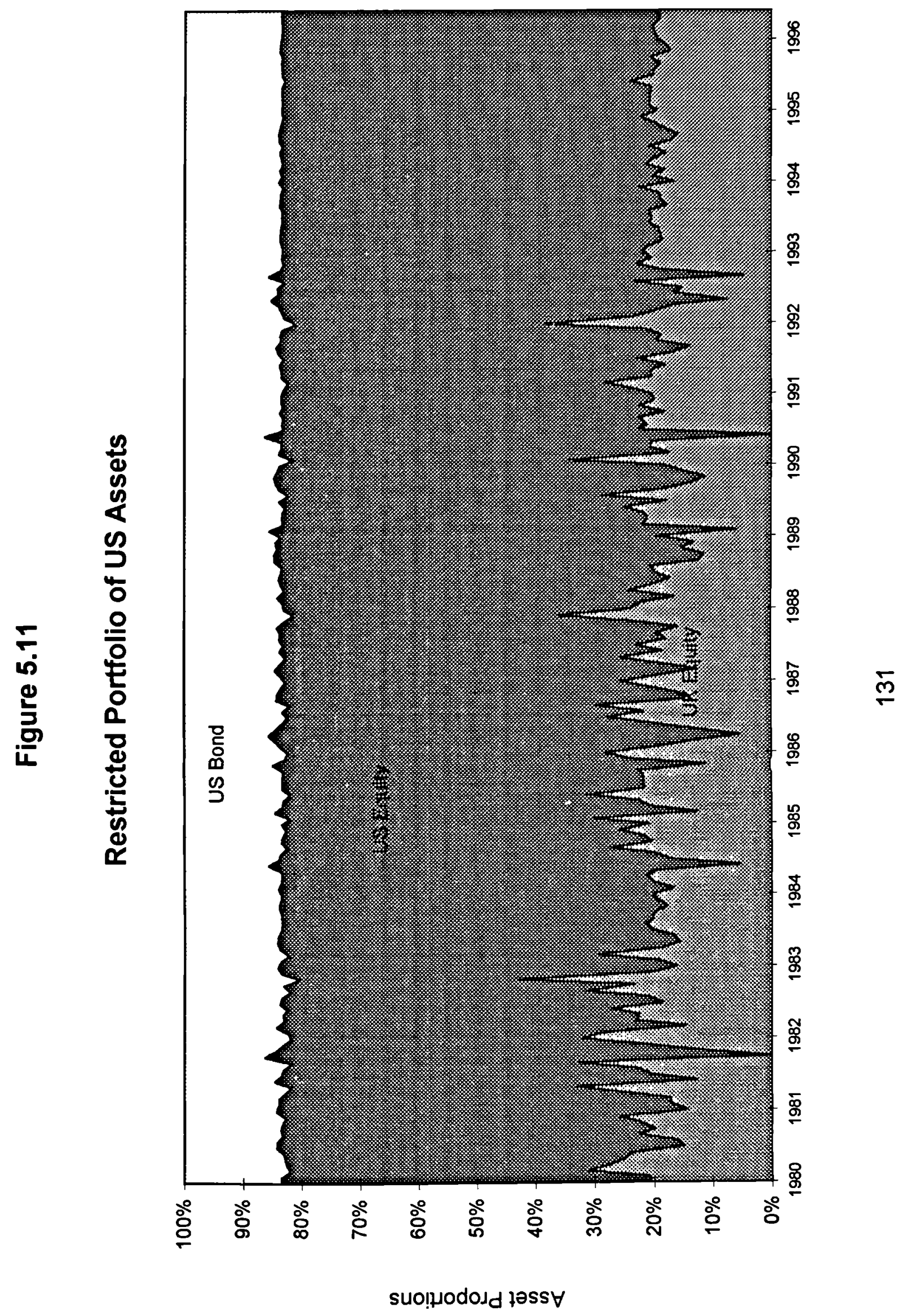




\section{Chapter 6}

\section{Global Asset Allocation}

\subsection{Introduction}

In this chapter we analyse the effects of increasing the number of assets in which a financial market participant may invest. In particular, we address the question from the perspective of a UK investor who is willing to internationally diversify his portfolio of risky financial assets. In preceeding chapters, we employ a multivariate GARCH model to generate time-varying covariance matrices of asset excess returns and use these estimated variances and covariances as inputs into the portfolio selection problem. One potential criticism of these optimal allocation models is that, due to the well documented problems of dimensionality in estimating M-GARCH models, the number of potential assets that an investor was allowed to hold was small - 
three in each of the aforementioned chapters. Here, we seek to address this shortcoming by allowing the investor to form a global bond portfolio and a European equity portfolio and then use these portfolios as one asset in the final allocation procedure.

The first step in our analysis forms an optimal portfolio of global bonds. Levy and Lerman(1988) find that a US investor who diversified across world bond markets could have realised returns more than twice the mean rate of return on a domestic US bond portfolio at the same risk level. In this chapter, we allow the UK investor to form an optimal portfolio of international government bonds. Having found the optimal time-varying asset proportions that form these portfolios in each period of the analysis, we calculate the realised returns on this optimal portfolio and use this series of returns as the returns on a single asset in the final allocation decision. A similar process is followed to form the optimal time-varying portfolio of European equities. Therefore the final portfolio selection decision focuses on five assets, namely UK equity, US equity, Japanese equity, European equity and a global bond.

We find that the optimal portfolio of bonds is dominated by the home bond with the Japanese bond also playing a significant role in its composition. There is also evidence that the excess returns on national government bonds may be more predictable than equity returns. The conditional second 
order moments are well characterised by a M-GARCH $(1,1)$ process. Meanwhile the most important component of the European equity portfolio is the French equity, followed by German equity with the Italian asset making a very limited contribution. Finally, we solve for the time-varying optimal global portfolios and discover that UK equity dominates the portfolio, with US and French equity being other substantial components. Both German equity and the UK bond also play a major role in forming these portfolios.

Our results also suggest that in the long run, volatility is transmitted between stock markets with the exception of Japanese and European markets but there is no evidence of any spillover effects between stock and bond markets. These markets appear to be segmented in the long run. In the short run, volatility spillovers again occur between the stock markets with Japan-Europe remaining the exception. We find limited evidence of volatility spillovers from the UK and Japan stock markets to bond markets but this is probably due to the fact that the bond portfolio is dominated by the government bonds of these countries.

Expanding our investment opportunity set is seen to offer the investor a significant risk-return advantage and substantially improve the performance of the optimal portfolio. However, the home bias problem discussed in the previous chapter is further accentuated by the introduction of more assets. Our results show that on average, $57 \%$ of investable funds should be held 
in home assets with the remaining $43 \%$ invested in foreign assets. However, actual survey results presented in French and Poterba(1991) claim that UK investors hold portfolios comprised of $82 \%$ home assets. This suggests that the problem is more acute than previously thought.

The remainder of the chapter is structured as follows. Section 6.2 presents the M-GARCH model that is used and discusses the data. In section 6.3 , we present the results of the analysis. Finally, section 6.4 contains our concluding remarks.

\subsection{The Model and Data}

\subsubsection{The Model}

The time-varying variances and covariances of the financial asset excess returns are generated using the parsimonious representation of the M-GARCH(1,1) model proposed earlier. This model has a VAR(1) structure in the conditional mean equations with the conditional second order moments following a $\operatorname{GARCH}(1,1)$ process. When we deal with national stock market indices, we allow for the possibility of including a dummy variable to capture the influence of the October 1987 Stock market crash. Therefore the model which we estimate is as follows: 


$$
\begin{aligned}
\mathbf{r}_{t}= & \boldsymbol{\alpha}+\boldsymbol{\beta} \mathbf{r}_{t-1}+\gamma d u m 87+\boldsymbol{\xi}_{t} \\
\boldsymbol{\xi}_{t} & \mid \Psi_{t-1} \sim N\left(0, \mathbf{H}_{t}\right) \\
\mathbf{H}_{t} & =\mathbf{V}^{\prime} \mathbf{V}+\mathbf{A}^{\prime}\left(\mathbf{H}_{t-1}-\mathbf{V}^{\prime} \mathbf{V}\right) \mathbf{A}+\mathbf{B}^{\prime}\left(\boldsymbol{\xi}_{t-1} \boldsymbol{\xi}_{t-1}^{\prime}-\mathbf{V}^{\prime} \mathbf{V}\right) \mathbf{B}
\end{aligned}
$$

where $\mathbf{r}_{t}$ represents a $k x 1$ vector of financial asset excess returns to be defined for each of the applications undertaken in this chapter. $\boldsymbol{\beta}$ is a $k x k$ matrix of regression parameters and $\boldsymbol{\gamma}$ is a $k x 1$ vector of parameters. $\mathbf{V}$, $\mathbf{A}$ and $\mathbf{B}$ are full $k x k$ symmetric matrices, with $\mathbf{V}^{\prime} \mathbf{V}$ denoting the long-run unconditional covariance matrix of asset excess returns. The parameters of the $\mathbf{A}$ and $\mathbf{B}$ matrices signify the importance of the short-run dynamics and identify the sources of these short-run deviations from the computed long-run value.

\subsubsection{The Data}

This chapter uses total return time series data on national stock market indices for the UK, the US, Japan, Germany, France and Italy and on government bond indices for the UK, the US, Japan and Germany. The UK equity market is represented by the Financial Times All share index, US equity by the Standard and Poors Composite index, while the new 
Tokyo stock exchange is used to represent Japanese equity. On the other hand, each of the European national indices is represented by a Datastream calculated index. We use these for European equities so that the sample period covered is as long as possible. Each of the national government bonds is represented by a Datastream calculated all government bond index. As the analysis is conducted from the perspective of a UK investor each of the national indices is converted to sterling using end of month exchange rates.

As in the previous chapters, the data used in this chapter is annualised monthly total returns for each asset including both capital gains and dividend payments in the case of equities and coupon payments for government bonds. The rate of return on the UK government 30 day Treasury bill is taken as the risk free rate of interest. All data was sourced from DATASTREAM.

The data covers a sample period beginning in January, 1982 and finishing in May, 1998. Again, we have chosen to work exclusively with rates of return in excess of the risk free rate to prevent volatility in the risk free rate from incorrectly contributing to the risk of the optimal risky portfolio. Once more, each of the excess return series were found to be stationary. 


\subsection{Results and Analysis}

\subsubsection{Global Bond Portfolio}

The model specified in equation (6.1) was maximised subject to the BHHH algorithm. In this application of the model, the vector of asset excess returns was defined as $\mathbf{r}=(u k b, u s b, g r b, j p b)^{\prime}$ with ukb denoting the UK government bond and usb, grb and jpb representing the bonds of the US, Germany and Japan respectively. The dummy variable for the stock market crash was omitted from this model.

This maximisation routine produced the following results with the corresponding $t$-statistics reported underneath in brackets. Since V, A and B are symmetric, we report only the lower triangle. 


\section{Mean Matrices}

$$
\boldsymbol{\alpha}=\left[\begin{array}{c}
2.94 \\
(1.73) \\
0.30 \\
(0.10) \\
-0.54 \\
(0.22) \\
-0.001 \\
(0.04)
\end{array}\right], \boldsymbol{\beta}=\left[\begin{array}{ccccc}
-0.01 & 0.02 & -0.20 & 0.14 \\
(0.15) & (0.36) & (2.71) & (2.22) \\
-0.17 & -0.04 & 0.37 & 0.04 \\
(1.19) & (0.42) & (2.86) & (0.38) \\
-0.09 & -0.04 & 0.32 & 0.02 \\
(0.75) & (0.53) & (2.83) & (0.24) \\
-0.13 & -0.16 & 0.48 & 0.15 \\
(0.76) & (1.62) & (3.75) & (1.69)
\end{array}\right]
$$

Covariance Matrices

$$
\mathbf{V}=\left[\begin{array}{llll}
23.73 & & & \\
(4.79) & & & \\
7.51 & 41.35 & & \\
(1.06) & (15.03) & & \\
5.04 & 12.26 & 26.54 & \\
(1.16) & (3.79) & (10.43) & \\
8.75 & 20.56 & 16.13 & 30.57 \\
(2.56) & (6.33) & (3.15) & (10.67)
\end{array}\right]
$$




$$
\mathbf{A}=\left[\begin{array}{cccc}
0.37 & & & \\
(1.35) & & & \\
-0.23 & 0.50 & & \\
(1.13) & (1.48) & \\
-0.56 & 0.07 & 0.29 & \\
(3.33) & (0.24) & (0.77) & \\
-0.63 & -0.47 & -0.43 & 0.09 \\
(4.14) & (2.18) & (1.80) & (0.35)
\end{array}\right], \mathbf{B}=\left[\begin{array}{ccccc}
0.18 & & & \\
(2.45) & & & \\
0.21 & 0.12 & & \\
(4.52) & (1.27) & & \\
-0.03 & 0.65 & 0.02 & \\
(0.50) & (4.06) & (0.23) & \\
-0.01 & 0.13 & -0.12 & 0.12 \\
(0.12) & (1.99) & (1.63) & (0.13)
\end{array}\right]
$$

\section{Discussion of Results}

The results of the estimation process are very interesting. Firstly, it would seem that the level of the excess returns for bonds are more predictable than for equity when compared to our other studies. In particular, the first lag of the excess return of the German bond has significant explanatory power over the current value of each government bond (looking at the third column of the $\boldsymbol{\beta}$ matrix). It has a positive relationship with each of the other bonds except with that of the UK. While the UK, US and German bonds have one statistically significant determinant, the excess return on the Japanese bond can also be predicted using its own lag and the lag of the US bond. 
Turning our attention to the second order moments, we notice that the elements of the long run matrix, V, are predominantly statistically significant. Each of the own variance elements is significant as are the covariance terms with the exception of those relating the UK bond to both the US and German bonds. However, when we focus on the short-run deviations about the long run, we find that most of these dynamics are coming from the off-diagonal elements. Each $(i, j) t h$ element of $\mathbf{H}_{t}$ has a corresponding statistically significant $(i, j)$ th element in either the $\mathbf{A}$ or $\mathbf{B}$ matrices. The relationships between each of the other bonds and the Japanese bond seem to be particularly important sources of short-run volatility with all the parameters in the fourth rows of $\mathbf{A}$ and $\mathbf{B}$ playing a crucial role in the transmission of volatility between international bond markets. The large number of significant covariance terms in the short run would suggest that the optimal portfolio will require frequent re-balancing.

The long-run covariance matrix $\left(\mathbf{V}^{\prime} \mathbf{V}\right)$ of government bond excess returns is

562.93

$178.16 \quad 1795.94$

$119.77 \quad 544.96 \quad 879.99$

$207.68 \quad 915.83 \quad 724.21 \quad 1694.03$ 
giving correlation coefficients between the excess returns over the riskfree rate on the UK bond and US bonds of $0.18, \mathrm{UK}$ and German bonds of 0.17 and UK and Japanese bonds of 0.21 . These correlations are quite low compared to the correlations between the foreign bonds and, recalling Markowitz's argument that we should diversify across assets with low return correlations, suggests that the UK bond should form a large part of the portfolio. The exchange rate component may partially explain the higher correlations between the non-domestic bonds.

\section{Optimal Portfolios}

The importance of short-run deviations from long-run volatility is captured for each of the bonds in figure 6-1. Here we plot the total conditional variance of each bond with its long-run value, depicted as a heavy horizontal line. For each bond, we observe that there is considerable short-run activity which is likely to influence our selection of the optimal bond portfolio in each period. Both the US and Japanese bonds are highly volatile though Japan has more short-run action. The returns on the German bond are surprisingly stable when we realise that this series also contains exchange rate risk. In fact, its range of movement is less than that of the UK bond, which is the home asset and is free from the effects of exchange rate movements. However, long-run volatility is smallest in the UK. 
Having computed the time-varying conditional variance-covariance matrices, we can now generate the portfolio frontiers and find the location and composition of the optimal bond portfolio for each period of the analysis. We first compute the optimal bond mix when there is no constraint on the sign of the assets in the portfolio, i.e. the investor is allowed to hold unlimited short positions in each of the assets. Under this allocation strategy, we find that the UK investor should hold the individual bonds as specified in figure 6-2. The UK bond is the dominant asset in the portfolio in each period, with a mean holding of $84 \%$. This is not surprising as it is free of exchange rate risk and is consistent with its relatively low correlation with the other assets. The next most popular bond is the Japanese bond, accounting for $17 \%$ of the portfolio on average. It contributed greatly to the composition of the portfolio in the early period of the sample but after 1996, its importance is diminished and is often held short. The US bond is the third most important and in the vast majority of periods has a small but positive holding, it has a mean position of $10 \%$. In contrast, the German bond is usually held short with the proceeds from this position allowing greater investment in the other bonds. On average its held short to the tune of $11 \%$ of investable funds. The main features of this allocation strategy are summarised in table 6.1 . 


\begin{tabular}{|l|c|c|c|}
\hline & Mean & Minimum & Maximum \\
\hline UK Bond & 84 & 56 & 106 \\
\hline US Bond & 10 & -30 & 31 \\
\hline German Bond & -11 & -62 & 28 \\
\hline Japanese Bond & 17 & -14 & 81 \\
\hline
\end{tabular}

Table 6.1: Summary statistics for the unrestricted global bond portfolio

As before, we find that this tactical asset allocation strategy produces asset holdings which are excessively volatile and require large revisions to the portfolio from period to period. In order to overcome the problem, we introduce an added restriction that the holdings of each asset have to be nonnegative. This constraint rules out the possibility of taking short positions in an asset which is consistent with the requirements of UK legislation. Figure 6-3 shows the composition of the optimal constrained global bond portfolio over the entire sample while table 6.2 summarises its key features.

\begin{tabular}{|l|c|c|c|}
\hline & Mean & Minimum & Maximum \\
\hline UK Bond & 80 & 69 & 94 \\
\hline US Bond & 6.5 & 0 & 13 \\
\hline German Bond & 0.5 & 0 & 5 \\
\hline Japanese Bond & 13 & 0 & 31 \\
\hline
\end{tabular}

Table 6.2: Summary statistics for the restricted global bond portfolio

Under this strategy, the UK bond continues to dominate the portfolio. On average, it accounts for $80 \%$ of the portfolio and its range of movement, $69 \%$ to $94 \%$, is substantially dampened. The relative importance of each of the other assets remains unaltered, with the Japanese bond having a 
mean holding of $13 \%$, followed by the US bond with $6.5 \%$ and the German bond contributing only $0.5 \%$ on average. Each of the foreign assets have a minimum holding of $0 \%$, proving that this added constraint is binding.

We now use these computed restricted weight vectors for each period of the analysis to compute the optimal portfolio of international government bonds. Once formed, this portfolio will constitute one asset in the final allocation procedure. Having found the optimal proportion of wealth to invest in national government bonds, we can then return to solve for the optimal amount of investment in each individual government bond when the opportunity to invest in equity also exists. This analysis is conducted in section 6.3.3.

\subsubsection{European Equity Portfolio}

We now build another asset based on a portfolio of European equities. All returns have been converted to sterling since we are addressing the problem from the perspective of a UK investor. Again we use the model as specified in equation (6.1) with the vector of excess returns defined as $\mathbf{r}=(\text { greq }, \text { freq }, \text { iteq })^{\prime}$ where greq, freq and iteq denote total returns on German, French and Italian equity market indices respectively. Surprisingly, the dummy variable for the 1987 stock market crash proved to be insignificantly different from zero and its omission helped the convergence proce- 
dure. Therefore, we omit this dummy variable from the model.

When this model achieved convergence under the $\mathrm{BHHH}$ algorithm, it yielded the following results. The results for each matrix defined in the model are presented below with the corresponding $t$-statistics in brackets underneath.

\section{Mean Matrices}

$$
\boldsymbol{\alpha}=\left[\begin{array}{l}
9.66 \\
(2.01) \\
12.56 \\
(2.30) \\
10.97 \\
(1.62)
\end{array}\right], \boldsymbol{\beta}=\left[\begin{array}{ccc}
-0.10 & 0.08 & 0.11 \\
(1.08) & (0.86) & (1.65) \\
-0.07 & 0.003 & 0.07 \\
(0.58) & (0.03) & (1.04) \\
-0.09 & 0.11 & 0.03 \\
(0.74) & (0.85) & (0.35)
\end{array}\right]
$$

\section{Conditional Covariance Matrices}

$\mathbf{V}, \mathbf{A}$ and $\mathbf{B}$ are all symmetric matrices. 


$$
\mathbf{V}=\left[\begin{array}{lll}
59.24 & & \\
(9.76) & & \\
54.82 & 39.46 & \\
(3.66) & (1.38) & \\
41.14 & 26.31 & 62.11 \\
(6.62) & (1.66) & (4.12)
\end{array}\right]
$$

$$
\mathbf{A}=\left[\begin{array}{lll}
0.02 & & \\
(0.03) & & \\
0.06 & 0.46 \\
(0.23) & (1.51) & \\
0.74 & -0.55 & 0.04 \\
(6.30) & (2.39) & (0.11)
\end{array}\right], \mathbf{B}=\left[\begin{array}{ccc}
0.11 & & \\
(1.13) & \\
0.32 & 0.11 \\
(3.64) & (1.11) & \\
0.14 & 0.01 & 0.08 \\
(1.83) & (0.12) & (1.21)
\end{array}\right]
$$

\section{Discussion of Results}

The results of this procedure confirm that its extremely difficult to predict the excess return on equity. It is clear from the $\boldsymbol{\beta}$ matrix that none of the lagged returns on the German, French and Italian stockmarkets have any statistically significant predictive power over their contemporaneous values. 
The exception might be the use of the first lag of the Italian return to forecast the German market but this is still only marginally different from zero.

Consistent with our other studies, we find that the conditional second order moments are much more forecastable. Looking at the $\mathrm{V}$ matrix, we see that all the long-run parameters are significantly different from zero, and very strongly so in most cases. This evidence alone is strongly supportive of the hypothesis that European equities exhibit (G)ARCH behaviour even at monthly horizons.

The long-run conditional covariance matrix of asset excess returns is given by:

$$
\left[\begin{array}{lll}
3509.65 & & \\
3247.90 & 4562.95 & \\
& & \\
2437.30 & 3293.59 & 6242.76
\end{array}\right]
$$

giving rise to large correlation coefficients between each pair of markets. As in the global bond portfolio selection (see section 6.3.1), our results suggest that in the short run, most of the actions stems from the asset covariance terms, i.e. the off-diagonal elements. From the $\mathbf{A}$ matrix, we find evidence that the relationships between Italian-German $\left(A_{31}\right)$ and ItalianFrench $\left(A_{32}\right)$ markets contribute significantly to the sources of short-run 
volatility in the system. Specifically, these parameters capture the persistence in this source of variability. Likewise, the B matrix has statistically significant elements in $B_{21}$ and $B_{31}$ which capture short-run relationships between the German-French markets and German-Italian markets respectively. Once more this indicates that re-balancing between these assets will be required in forming our optimal tactical asset allocations.

Our results are consistent with volatility transmissions across the major European markets in both the long run and the short run. This strong support of the importance of the covariance terms in computing both the total and long-run conditional variances and covariances is strongly supportive of our modelling approach. Many other studies assume such matrices to be diagonal (e.g. Engle et al.(1990) or adopt the restrictive constant correlations approach of Bollerslev(1990)) and we demonstrate that these studies lose a great deal of important information. Exploiting this information allows us to form better performing portfolios of risky assets.

\section{Optimal Portfolios}

Figure 6-4 shows the conditional variances of each asset together with their long-run unconditional values. Each asset exhibits a large degree of variability, though the French equity is distorted by one spike which coincides with the US stock market crash. Both the German and Italian markets were 
effected to a lesser degree but have other periods of turbulence also. When we focus on the long-run variances, we see that German market is the least volatile of the European markets while the Italian market is highly volatile.

As before, we begin with an unrestricted allocation of funds among the three asset classes. The optimal mix of assets for each period of the analysis is captured in figure 6-5. Clearly from this picture, we see that the French equity dominates the portfolio. It has a mean holding of $66.5 \%$ of investable funds. The French equity is always held in positive quantities. The German equity, on the other hand, has an average long position of $34.5 \%$ but its holding is quite volatile and in fact, its held short in at least 10 periods. The Italian equity holding is most often quite small in magnitude and on average is held short, composing $-1 \%$ of the portfolio. Table 6.3 summarises this asset allocation strategy.

\begin{tabular}{|l|c|c|c|}
\hline & Mean & Minimum & Maximum \\
\hline France & 66.5 & 15 & 191 \\
\hline Germany & 34.5 & -71 & 90 \\
\hline Italy & -1 & -19 & 29 \\
\hline
\end{tabular}

Table 6.3: Summary statistics for unrestricted European equity portfolio

Once more, this asset allocation is deemed to be excessively volatile, so we adopt the alternative approach where short sales are prohibited. Under this strategy, we find an allocation that is very stable over time. Figure 
6-6 depicts the constituents of this portfolio over the entire sample. The portfolio consists mainly of French equity in each period and on average contains $66 \%$ of this asset. It is quite consistent and always contributes between $65 \%$ and $75 \%$ of the portfolio. The German equity comprises most of the remainder of the portfolio, having a mean holding of $31.5 \%$. There are a number of occasions when the added restriction is binding and the German equity is not held. Its holding never exceeds $34 \%$ of investment funds. Finally, the Italian equity is not a very popular asset from the perspective of a UK investor. It is very often omitted from the optimal portfolio of European equities and has a mean holding of $2.5 \%$. The returns on Italian stock are obviously not sufficient to compensate the investor for holding this highly volatile asset. This problem is compounded by the fact that the Italian lira is also a volatile currency and the exchange rate risk is likely to be larger than that for the sterling-mark or sterling-franc rate. Table 6.4 presents a summary of this restricted allocation strategy.

\begin{tabular}{|l|c|c|c|}
\hline & Mean & Minimum & Maximum \\
\hline France & 66 & 65 & 75 \\
\hline Germany & 31.5 & 0 & 34 \\
\hline Italy & 2.5 & 0 & 25 \\
\hline
\end{tabular}

Table 6.4: Summary statistics for restricted European equity portfolio

We use these restricted time-varying weights to form the optimal port- 
folio of European equity which will constitute a single asset in the global asset allocation presented in the following sub-section.

\subsubsection{Global Asset Allocation}

Having formed optimal portfolios of bonds and European equities, we can now address the question of global asset allocation. As before, we estimate the time-varying covariances using our M-GARCH model of equation (6.1). Here the vector of asset excess returns is given by $\mathbf{r}=(u k e q, u s e q, j p e q, e u e q, g b l b)^{\prime}$ where ukeq, useq and jpeq refer to the national stock market returns for the UK, US and Japan respectively; eueq denotes the portfolio of European equities formed using the time-varying vector of asset weights generated by the results of section 6.3 .2 ; while gblb refers to the global bond portfolio formed on the basis of our results from section 6.3.1. Once more, the model was maximised subject to the $\mathrm{BHHH}$ algorithm and the parameter estimates are reported here with corresponding $t$-statistics in parentheses. 
Mean Matrices

$\boldsymbol{\alpha}=\left[\begin{array}{c}11.74 \\ (2.78) \\ 11.77 \\ (1.96) \\ 4.98 \\ (0.61) \\ 14.25 \\ (2.44) \\ 2.88 \\ (1.12)\end{array}\right], \boldsymbol{\beta}=\left[\begin{array}{cccccc}0.03 & 0.07 & 0.07 & -0.18 & 0.22 \\ (0.31) & (0.64) & (1.11) & (2.12) & (0.96) \\ 0.03 & 0.04 & 0.03 & -0.12 & 0.05 \\ (0.19) & (0.30) & (0.41) & (1.04) & (0.17) \\ -0.002 & 0.01 & 0.11 & -0.10 & 0.31 \\ (0.02) & (0.05) & (1.11) & (0.65) & (0.80) \\ -0.11 & 0.13 & 0.05 & -0.06 & 0.17 \\ (0.88) & (1.02) & (0.67) & (0.57) & (0.56) \\ 0.03 & -0.01 & 0.03 & -0.09 & 0.10 \\ (0.41) & (0.30) & (0.91) & (2.00) & (0.95)\end{array}\right], \gamma=\left[\begin{array}{c}(1.67) \\ -160.54 \\ (1.85) \\ 0 \\ 0 \\ 0 \\ 0\end{array}\right]$


Covariance Matrices

$\mathbf{V}=\left[\begin{array}{cccccc}51.72 & & & & \\ (7.36) & & & & \\ 31.78 & 53.46 & & & \\ (2.81) & (9.54) & & & \\ 24.66 & 25.54 & 74.83 & & \\ (1.95) & (1.93) & (5.66) & & \\ 30.37 & 13.12 & 11.99 & 46.96 & \\ (4.37) & (1.68) & (0.80) & (4.17) & \\ 13.51 & 3.88 & 4.84 & -2.31 & 0.07 \\ (0.94) & (0.16) & (0.09) & (0.03) & (1.50)\end{array}\right]$


$\mathbf{A}=\left[\begin{array}{rrrrrr}-0.06 & & & & \\ (0.17) & & & & \\ -0.45 & -0.53 & & & \\ (1.77) & (2.17) & & & \\ -0.03 & -0.16 & -0.17 & & \\ (0.07) & (1.63) & (0.25) & & \\ -0.41 & -0.15 & 0.13 & 0.59 & \\ (1.90) & (0.52) & (0.33) & (2.39) & \\ 0.04 & -0.01 & 0.43 & -0.25 & -0.70 \\ (0.09) & (0.02) & (1.16) & (1.04) & (1.77)\end{array}\right]$ 


$$
\mathbf{B}=\left[\begin{array}{cccccc}
0.28 & & & & \\
(1.96) & & & & \\
-0.09 & 0.14 & & & \\
(0.84) & (1.08) & & & \\
0.18 & 0.05 & 0.11 & & \\
(2.42) & (0.63) & (1.12) & & \\
0.09 & 0.17 & -0.07 & 0.20 & \\
(0.95) & (1.76) & (0.94) & (1.76) & \\
0.10 & -0.02 & 0.12 & -0.03 & -0.04 \\
(1.69) & (0.21) & (2.35) & (0.51) & (0.41)
\end{array}\right]
$$

\section{Discussion of Results}

The coefficients of the $\operatorname{VAR}(1)$ conditional mean equation confirm the difficulty of predicting future financial asset excess returns. The majority of these coefficients are not statistically different from zero. The exception is that the first lag of the excess return on European equity seems to have some predictive power over both UK equity returns and returns on the global bond. Interestingly, the relationship between the European equity and each of the other assets is negative, though only in the case of UK equity and the global bond (which is dominated by the UK bond) is the relationship statistically significant at the $5 \%$ confidence level. It was found to aid the 
convergence procedure to include a dummy variable for the October 1987 stock market crash in the equations for both UK and US equities.

Focusing on the long-run covariance matrix, we find that $\mathrm{V}$ has statistically significant elements along the main diagonal and among the covariance terms between the individual equity markets. Focusing on the covariance terms in rows 1 to 4 of this matrix strongly supports the hypothesis that volatility is transmitted from one equity market to another. This volatility spillover is evidenced between all pairs of markets with the exception of Japan-Europe whose markets appear to be segmented from each other. However, the off-diagonal elements of the final row are not statistically different from zero, suggesting that in terms of the transmission mechanism for long-run volatility, bond markets are segmented from equity markets. This suggests that there is no spillover of stockmarket volatility to national government bond markets in the long run.

Turning our attention to sources of short-run volatility, we find that there is evidence of contagion effects between most of the stockmarkets across the world. Evidence of short-run volatility spillovers between UK and US is supplied by the statistical significance of parameter $A_{21}, \mathrm{UK}$ and European spillovers by $A_{41}$, UK and Japanese by $B_{31}$, US and Japanese by $A_{32}$ and US and European by $B_{42}$. Only European and Japanese markets seem to be segmented (as in the long-run situation) with neither $A_{43}$ nor $B_{43}$ being 
significantly different from zero. There is also evidence of the importance of short-run deviations from long-run volatility by virtue of the significance of the diagonal elements for the UK $\left(B_{11}\right)$, the US $\left(A_{22}\right)$ and European equity markets $\left(A_{44}\right.$ and $\left.B_{44}\right)$.

Finally in the short-run, we find some evidence that stock and bond market volatility may be linked. There is a statistically significant relationship between our global bond portfolio and both the UK and Japanese equity markets. This may be due to the fact that our global bond portfolio is dominated by UK and Japanese bonds (on average these two bonds comprise $93 \%$ of the portfolio). Short-run deviations in bond market volatility seem to be caused by its own variance effect and the aforementioned relationships with UK and Japanese equity markets. Consequently we would expect much of the portfolio re-balancing to occur between the individual equities.

The long-run variance-covariance matrix of asset excess returns is given by:

$$
2674.76
$$

1643.513867 .95

$1275.12 \quad 2148.83 \quad 6859.04$

$1570.81 \quad 1666.50 \quad 1981.23 \quad 3443.66$

$\begin{array}{lllll}699.15 & 636.92 & 794.73 & 411.08 & 226.58\end{array}$


and shows that when all returns are denominated in sterling, the Japanese equity market is the most volatile with the UK market being the most tranquil. The global bond is by far the least volatile asset available to the potential investor. Figure 6-7 plots the unconditional variance of each asset together with short-run deviations about this value. Clearly, the short-run action is going to be important in determining the optimal mix of assets in our time-varying portfolios.

\section{Portfolio Frontiers}

Having computed the conditional covariance matrices for each period of the analysis, we use these variances and covariances as inputs into the meanvariance portfolio selection process. We then generate the portfolio frontiers for each period of the analysis. Figure 6-8 contains information on the distribution of the frontiers by plotting the time-varying mean, minimum and maximum frontiers for the entire sample. Firstly, we observe that there has been considerable variation in both the shape and location of the frontier. This alone suggests that we have gained vital information by adopting our modelling approach as opposed to assuming a static unconditional covariance matrix. Again, we observe positive skewness in the distribution suggesting that there are some periods in which risk is far higher than the mean. 
Secondly, we plot the mean frontier generated by our two studies on international diversification. In the previous chapter, we allow the UK investor only one opportunity to diversify internationally. The only foreign asset in the investment opportunity set is US equity. Here we extend the number of foreign assets to include equity and government bonds from the US, Japan, and Germany as well as French and Italian equity. This allows the UK investor to select the optimal portfolio from among 10 risky financial assets including 8 foreign assets. Figure 6-9 plots the mean frontier generated by these two investment opportunity sets. We see that the UK investor can reap substantial risk-return gains from considering a broader range of potential investment vehicles. This is clear from the fact that the frontier generated by the "global" investment set is closer to the origin at all points, thereby delivering equal return for the burden of less risk. It is therefore possible for investors to reduce their portfolio risk by holding a broader range of non-domestic assets. Figure 6-10 confirms this fact by plotting not only the mean frontiers but also the minimum and maximum frontiers. The global frontiers (shown by the heavy lines) always lie inside their counterparts generated by the more restricted investment set. 


\section{Optimal Portfolio Performance}

With the portfolio frontier computed for each period, we can identify the optimal portfolio of risky assets as the point of tangency between the capital market line and the frontier. Figure 6-11 shows the behaviour of both the excess return and the standard deviation of the optimal portfolio over time. As expected they move together, showing that the burden of more risk must be compensated by a higher return.

Calculating the Sharpe Performance Index $\left(S P I_{t}=\frac{\text { return }}{r i s k_{t}}\right)$ for each portfolio, gives the greatest confirmation that the extended investment set offers an improved portfolio to the investor. Figure 6-12 shows the SPI over the whole sample. On average, the optimal portfolio has a SPI value of 0.30. This represents a massive increase of $67 \%$ over the average SPI value achieved when the only foreign asset was US equity and an even bigger increase of $87.5 \%$ when the investment set was limited to domestic assets. Therefore, the message is clear. International diversification leads to better performing portfolios and the greater the number of assets considered, the larger the potential gains in performance terms.

\section{Optimal Portfolio Composition}

We begin our asset allocation problem by looking at the unrestricted allocation strategy. There are no constraints on the sign of the asset holdings. 
Using this approach, we get a highly volatile series of asset weights for each of the five assets in the analysis. Figure 6-13 shows their behaviour over time. As usual, the home equity dominates the portfolio with an average holding of $52 \%$. However, there is a period when this asset is held short. In fact each of the assets in the analysis is sold short at some stage, leading to large and frequent changes in the composition of the portfolio. The US equity is the next most important asset, accounting on average for $28 \%$ of investable funds. Both the European equity and the global bond have positive mean holdings with $20 \%$ and $11 \%$ of the portfolio respectively. However, the Japanese equity is frequently held short and on average has a position of $-11 \%$. However, the allocation strategy yields extremely volatile holdings and these are summarised in table 6.5.

\begin{tabular}{|l|c|c|c|}
\hline & Mean & Minimum & Maximum \\
\hline UK Equity & 52 & -11 & 305 \\
\hline US Equity & 28 & -21 & 216 \\
\hline Japanese Equity & -11 & -45 & 5 \\
\hline European Equity & 20 & -47 & 142 \\
\hline Global Bond & 11 & -353 & 62 \\
\hline
\end{tabular}

Table 6.5: Summary statistics for unrestricted global asset portfolio

This strategy is clearly not implementable in a world where there are transaction costs incurred when changing your portfolio, both monetary and time costs. Therefore, it seems more necessary than ever to adopt our 
constrained allocation procedure which prohibits the short sale of assets. Figure 6-14 shows the composition of this optimal portfolio over the entire sample. The UK equity again dominates with a mean holding of $52 \%$ and even though its volatility is reduced, it still moves between a range of 0 and $90 \%$. With the investor no longer able to generate extra funds through the short sale of Japanese equity, the European equity replaces the US equity as the second most important asset, with $21 \%$ of the portfolio as against $20 \%$. However, when we break the European equity down into its component parts, the US equity regains its status as the most attractive foreign asset. The Japanese equity is almost always omitted from the optimal portfolio and has a mean position of zero. Even when it makes a fleeting appearance, it never accounts for more than $4 \%$ of the portfolio. The global bond holding is the most smooth in the portfolio. This is confirmed by the relatively small range of movement throughout the 16 year sample, from $5 \%$ to $8 \%$. The investor seems to be keeping, on average, $7 \%$ of investable funds in the safest asset and rarely alters its position. This is consistent with the non-significance of the covariance terms linking government bonds to equity markets. Table 6.6 summarises the asset holdings.

We now proceed to decompose the European equity and the global bond into their constituent parts (see figures 6-3 and 6-6). Since the French equity 


\begin{tabular}{|l|c|c|c|}
\hline & Mean & Minimum & Maximum \\
\hline UK Equity & 51.8 & 0 & 90 \\
\hline US Equity & 20 & 0 & 56 \\
\hline Japanese Equity & 0 & 0 & 4 \\
\hline European Equity & 21.2 & 0 & 142 \\
\hline Global Bond & 7 & 5 & 8 \\
\hline
\end{tabular}

Table 6.6: Summary statistics for restricted global asset portfolio

dominated the European portfolio, it becomes a very important component of the global asset portfolio and contributes $14 \%$ on average. German equity also plays an important role in the portfolio, with an average holding of almost $7 \%$. The Italian equity, on the other hand, does not hold much appeal to the UK investor and has a mean holding of only $0.5 \%$.

Looking at the bond portfolio, we found that it is mainly composed of UK and Japanese bonds. In the global asset portfolio, these assets account for almost $6 \%$ and $1 \%$ of the portfolio respectively. Interestingly, the smooth allocation to bonds, results in the UK bond being the only asset that is always held in the portfolio. Neither US nor German bonds contribute significantly to the portfolio with mean holdings of $0.5 \%$ and $0 \%$. In table 6.7, we combine all the parts of this study and show the mean and range of asset holdings that constitute the optimal portfolio of assets available to a UK investor. 


\begin{tabular}{|l|c|c|c|}
\hline & Mean & Minimum & Maximum \\
\hline UK Equity & 51.7 & 0 & 90.3 \\
\hline US Equity & 20.0 & 0 & 56.5 \\
\hline Japanese Equity & 0.0 & 0 & 4.1 \\
\hline French Equity & 14.0 & 0 & 47.3 \\
\hline German Equity & 6.7 & 0 & 25.3 \\
\hline Italian Equity & 0.5 & 0 & 14.6 \\
\hline UK Bonds & 5.6 & 4.1 & 7.1 \\
\hline US Bonds & 0.5 & 0 & 1.0 \\
\hline German Bonds & 0 & 0 & 0.3 \\
\hline Japanese Bonds & 1.0 & 0 & 2.6 \\
\hline
\end{tabular}

Table 6.7: Global Asset Allocation for UK investor

These results clearly show that the degree of home country bias is greater than estimated in the previous chapter. When we allowed the UK investor the opportunity to invest in US equity and domestic assets only, our results showed that $75 \%$ of the portfolio should on average be held in home assets. Survey results showed that the actual holding was approximately $82 \%$ (French and Poterba(1991)), which suggested that the degree of home bias in the UK was not acute and certainly not as large as in the US. However, when we expand the number of foreign assets which the investor may potentially hold, we find that the home bias is quite large. Our results suggest that, on average, the domestic investor should upto $43 \%$ of the optimal portfolio in non-UK assets. The mean portfolio is comprised of 8 assets, six of which are foreign. Therefore, it is clear that increased international diversification on the part of UK investors would yield portfolios with better performance and more particularly portfolios which delivered a more 
attractive return for each level of risk.

\subsection{Conclusion}

This chapter sought to build a truly optimal global portfolio of risky financial assets from the perspective of a UK investor. We undertake this analysis using the parsimonious parameterisation of the M-GARCH(1,1) model presented in this thesis. We overcome the problem of having too few assets in our selection procedure by first forming a portfolio of international government bonds. This portfolio enters the final allocation problem as a

single asset. A similar process is adopted for European equity markets. In the final allocation, we have five assets, UK, US, Japanese and European equity as well as our global bond. The proportions of wealth allocated to investment in both European equity and the global bond were then further broken down into their constituent components.

Our results generate many points worthy of attention. Firstly, concentrating on the restricted allocation, the optimal portfolio is dominated by the home equity for the UK investor. Also the UK bond is an important investment vehicle and in fact this is the only asset which is never omitted from the portfolio. However, on average, these two assets constitute only $57 \%$ of the portfolio, thereby showing that non-UK assets should account for the remaining $43 \%$. In the mean optimal portfolio six foreign assets 
are held in positive quantities along with the two domestic assets. US, French and German equity together make up $40 \%$ of the portfolio. These holdings are in stark contrast to observed investment behaviour and when compared with the survey results of French and Poterba(1991), who show UK investors hold $18 \%$ of their portfolios in international assets, the degree of home bias inherent in the UK market is very substantial. Furthermore, our results mean that increased levels of international diversification would benefit the UK investor.

Secondly, using the expanded set of investment opportunities presented in this chapter, we find that our optimal portfolios have much larger SPI values than those where international diversification is limited to a single asset, i.e. US equity. On average, the SPI values were $67 \%$ higher, 0.30 versus 0.18 . The gains in SPI values are even greater $(87.5 \%)$ when compared to the portfolios in which the investor was limited to holding only domestic assets. The advantages of increasing the investment set can also be seen by comparing the mean portfolio frontiers generated by the two studies of international diversification. Introducing more assets shifts the frontier significantly towards the origin and offers the investor the opportunity to enjoy higher levels of return for each risk level.

Finally, our results show that in the long run, volatility is transmitted between individual bond markets and stock markets. However, bond 
markets appear to be segmented from stock markets. There is no evidence of volatility spillover effects between stock and bond markets. Also, the Japanese and European stock markets appear to be unaffected by volatility in the other, though there is strong evidence of spillovers between all other pairs of equity markets. Likewise, with regards to short-run volatility, we find evidence of stockmarket spillovers for all pairs of equity markets excluding the Japanese-European combination. However, there is some support for short-run bond market volatility being influenced by UK and Japanese stock markets. This is probably due to the fact that our global bond is largely dominated by the bonds of these two nations.

In summary, volatility sources for bonds and stocks appear to be different. Therefore, from a diversification point of view, its beneficial to hold both in a portfolio of risky assets. Our results suggest that improved portfolio performance can be derived by diversifying across international markets and that the greater the number of assets considered, the greater the benefits accruing to the investor. Finally, while the home assets dominate the portfolio, our results suggest that an excessive degree of home country bias exists when choosing portfolios of risky assets and hence unexploited opportunities remain. 
Figure 6.1

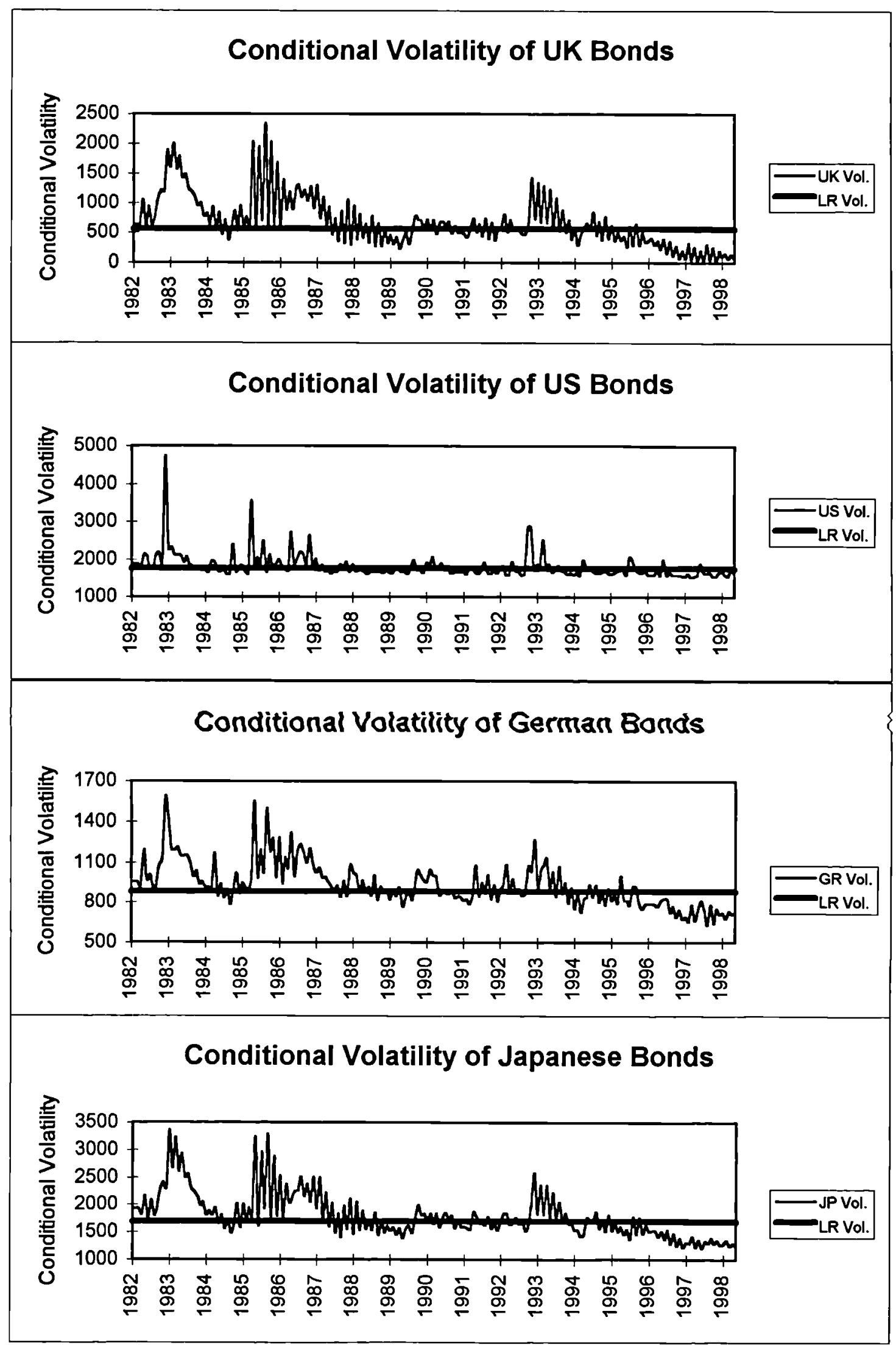




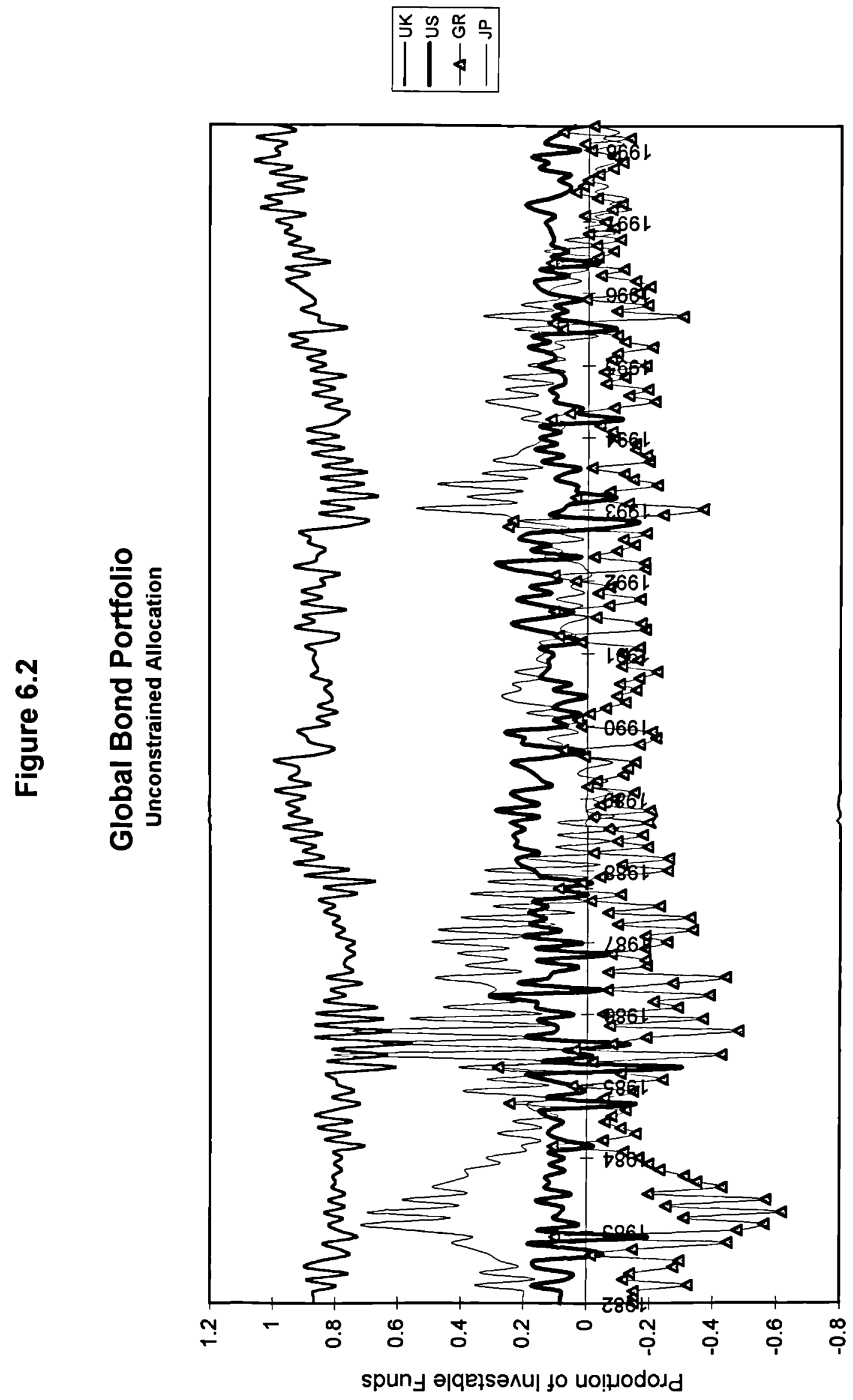

$\stackrel{ }{r}$ 


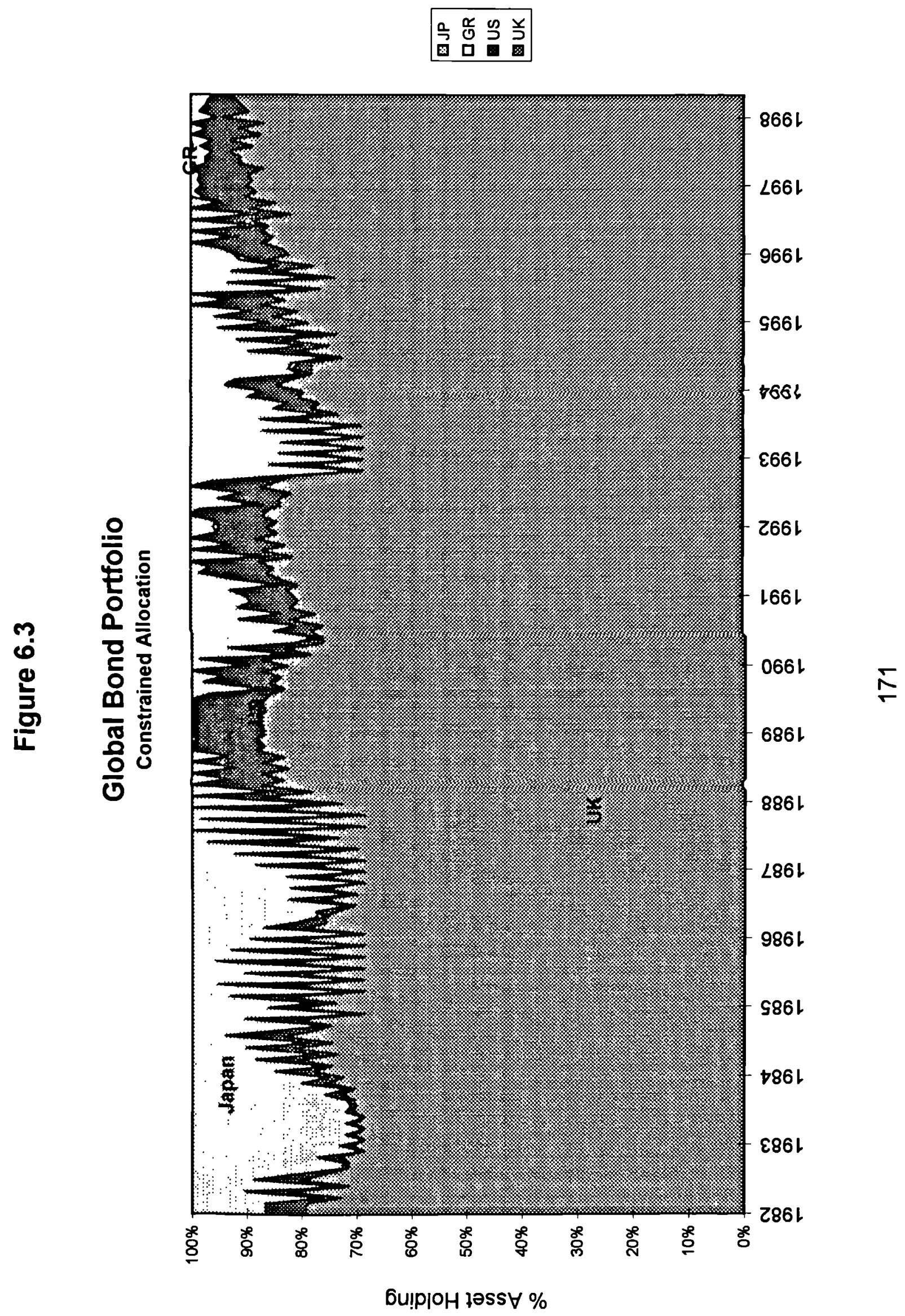


Figure 6.4

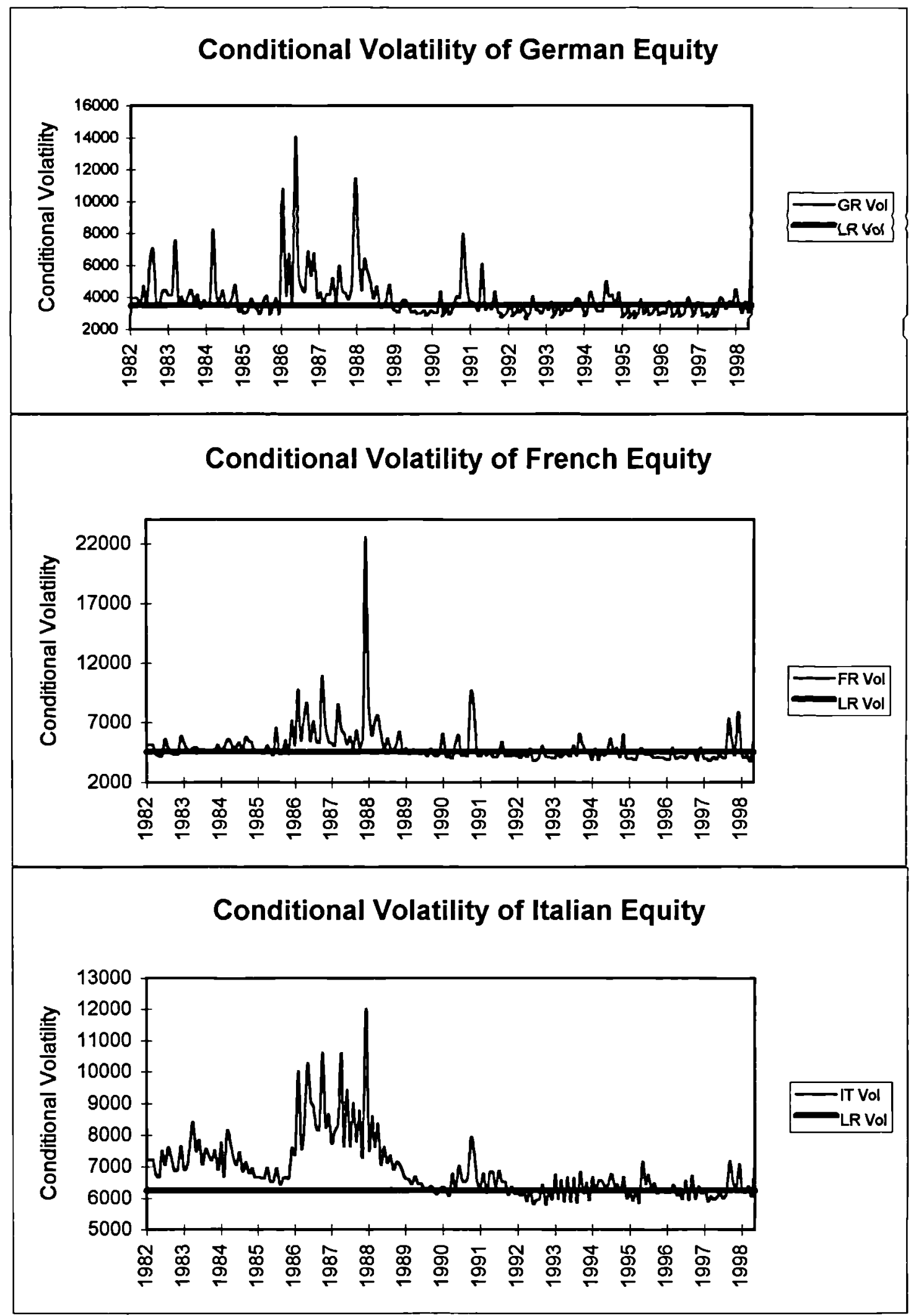



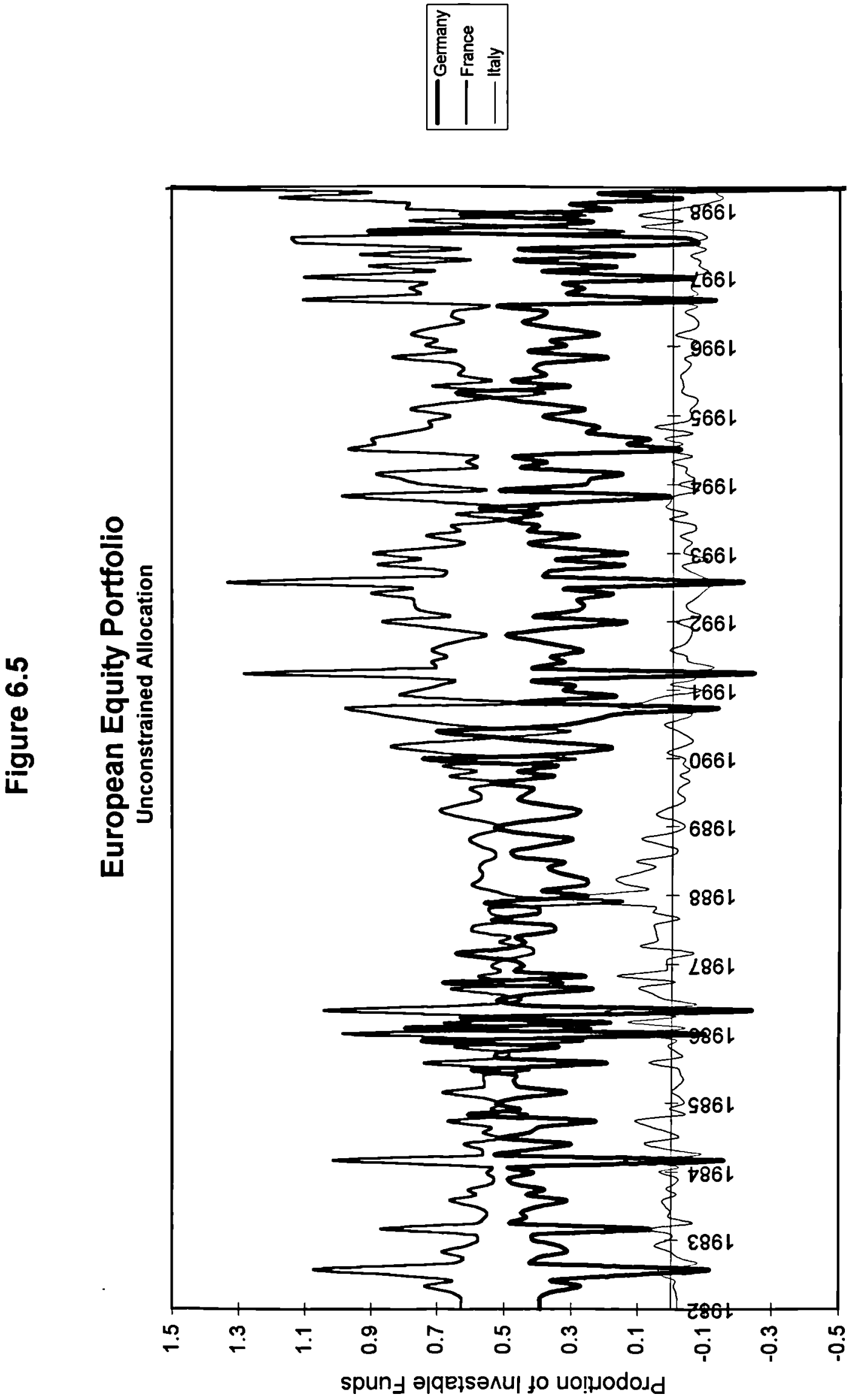

$\stackrel{M}{N}$ 


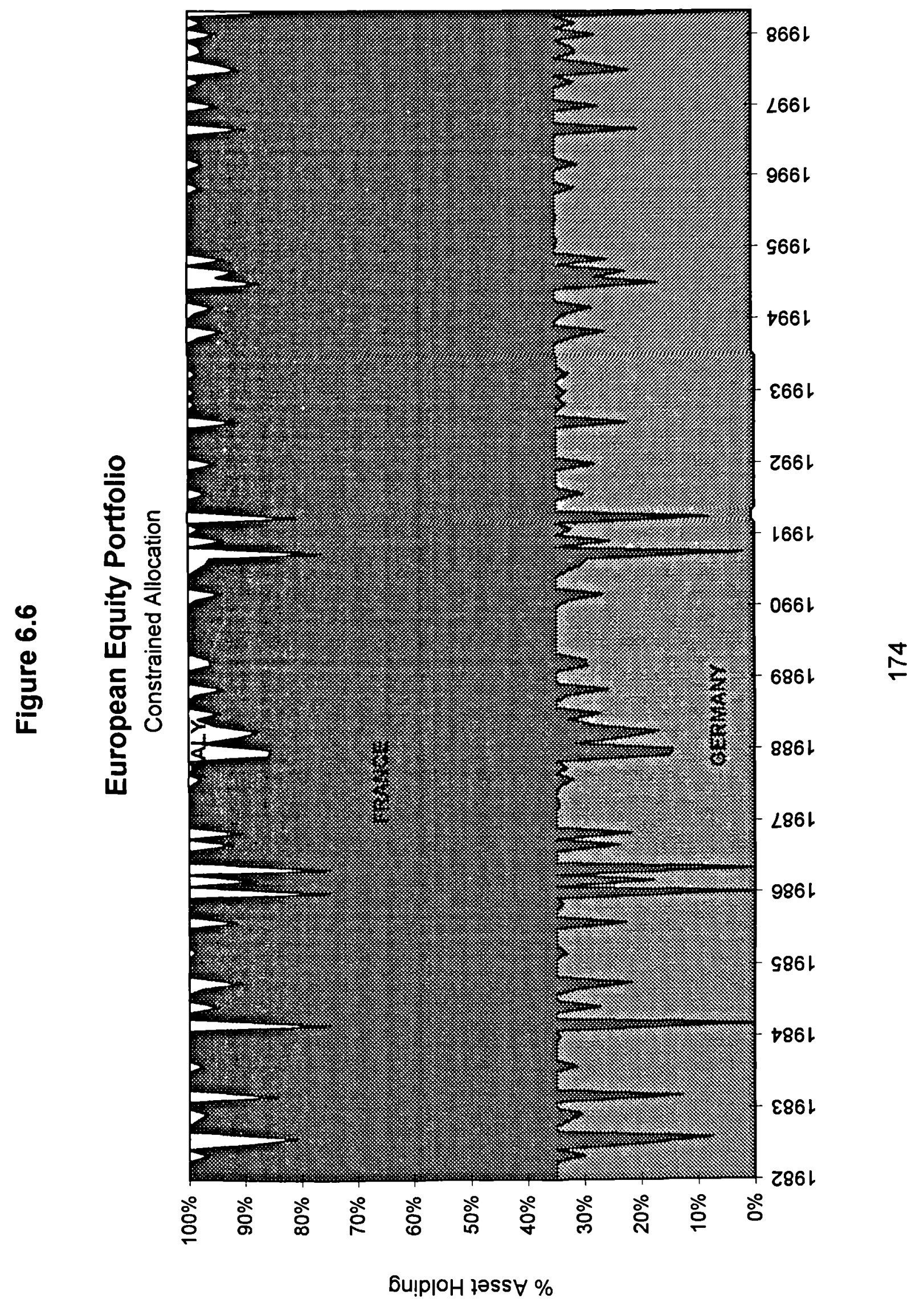


Figure 6.7

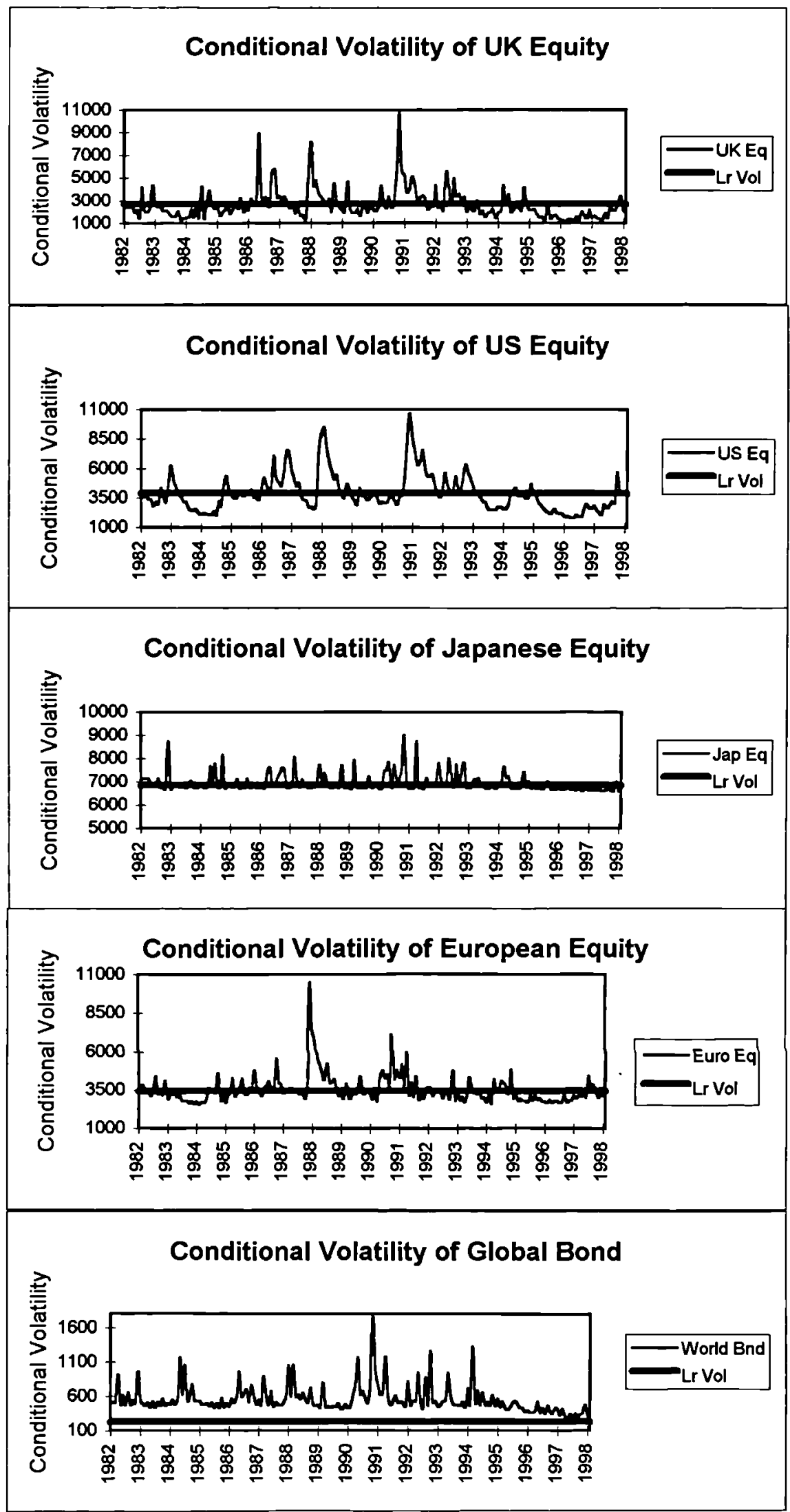




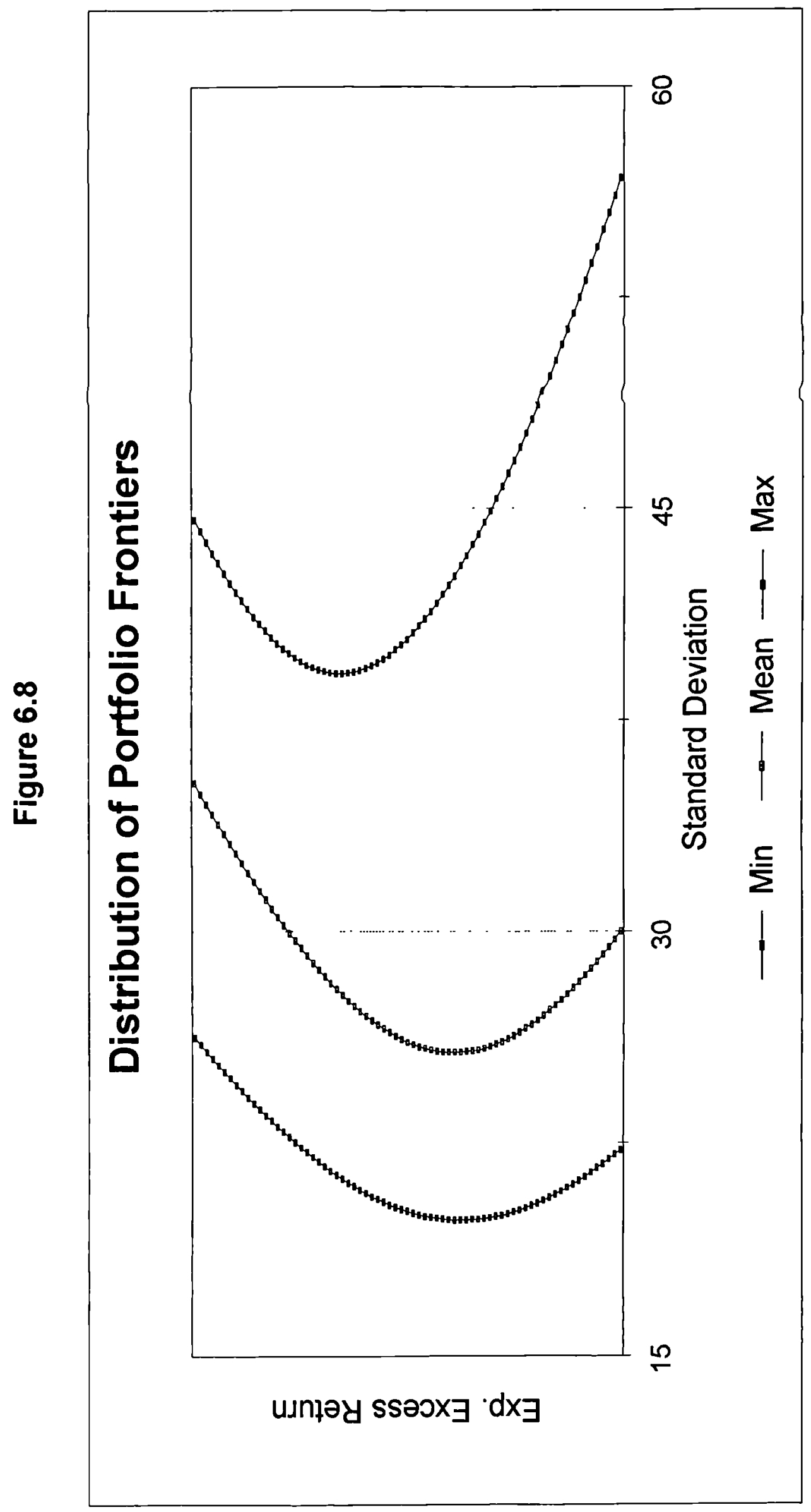

$\stackrel{0}{ }$ 


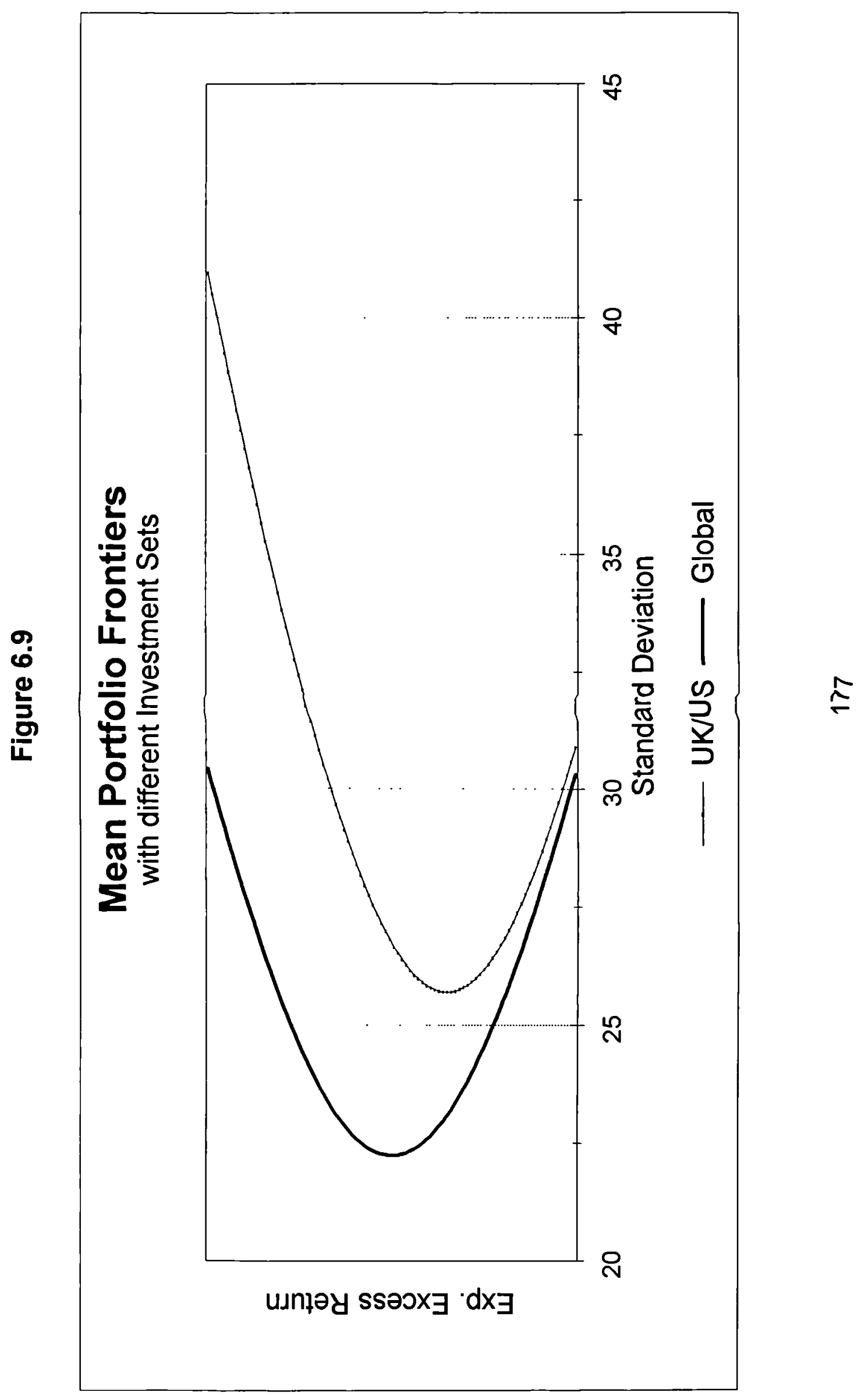




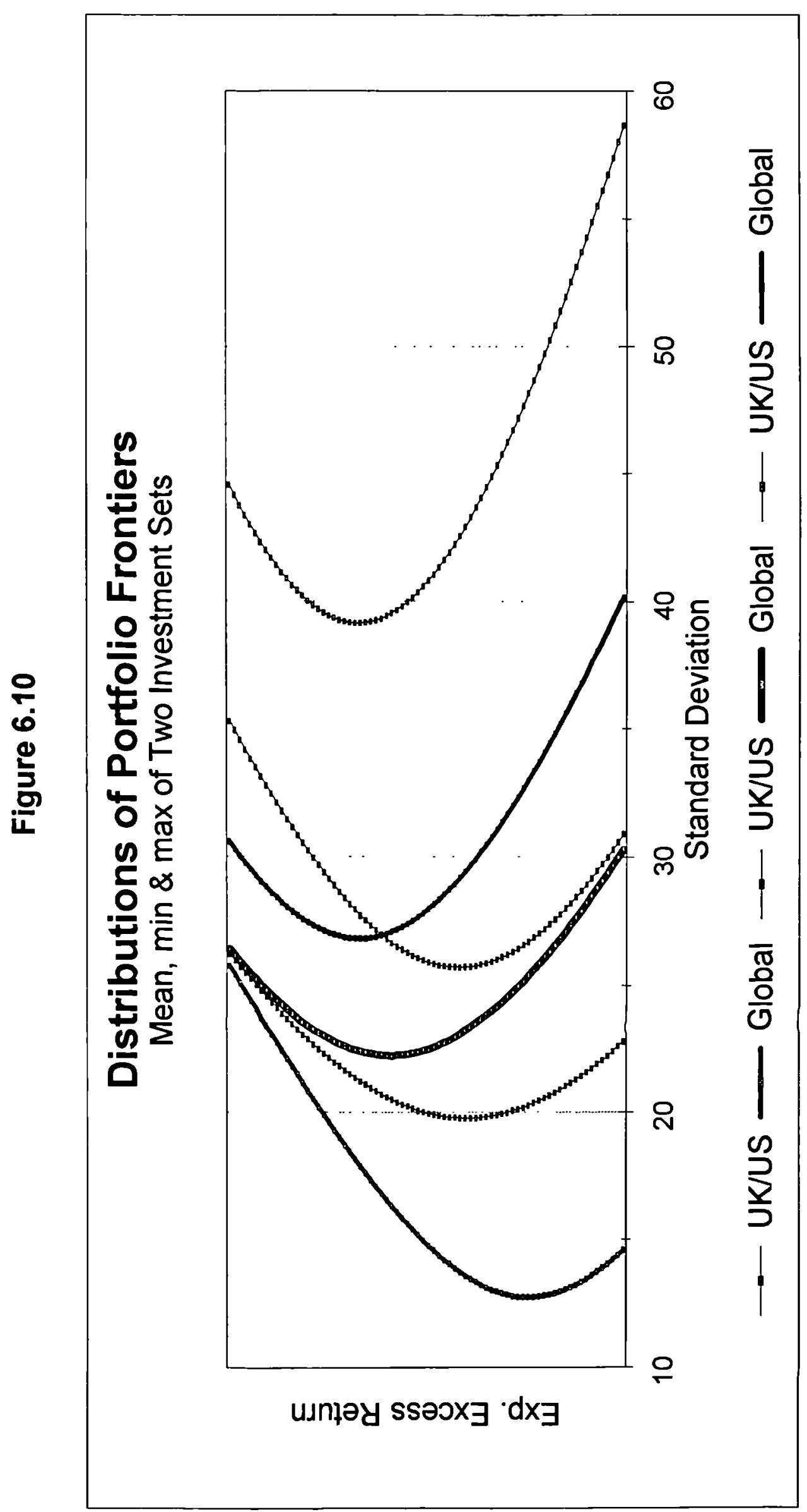

$\stackrel{\infty}{\sim}$ 
Figure 6.11

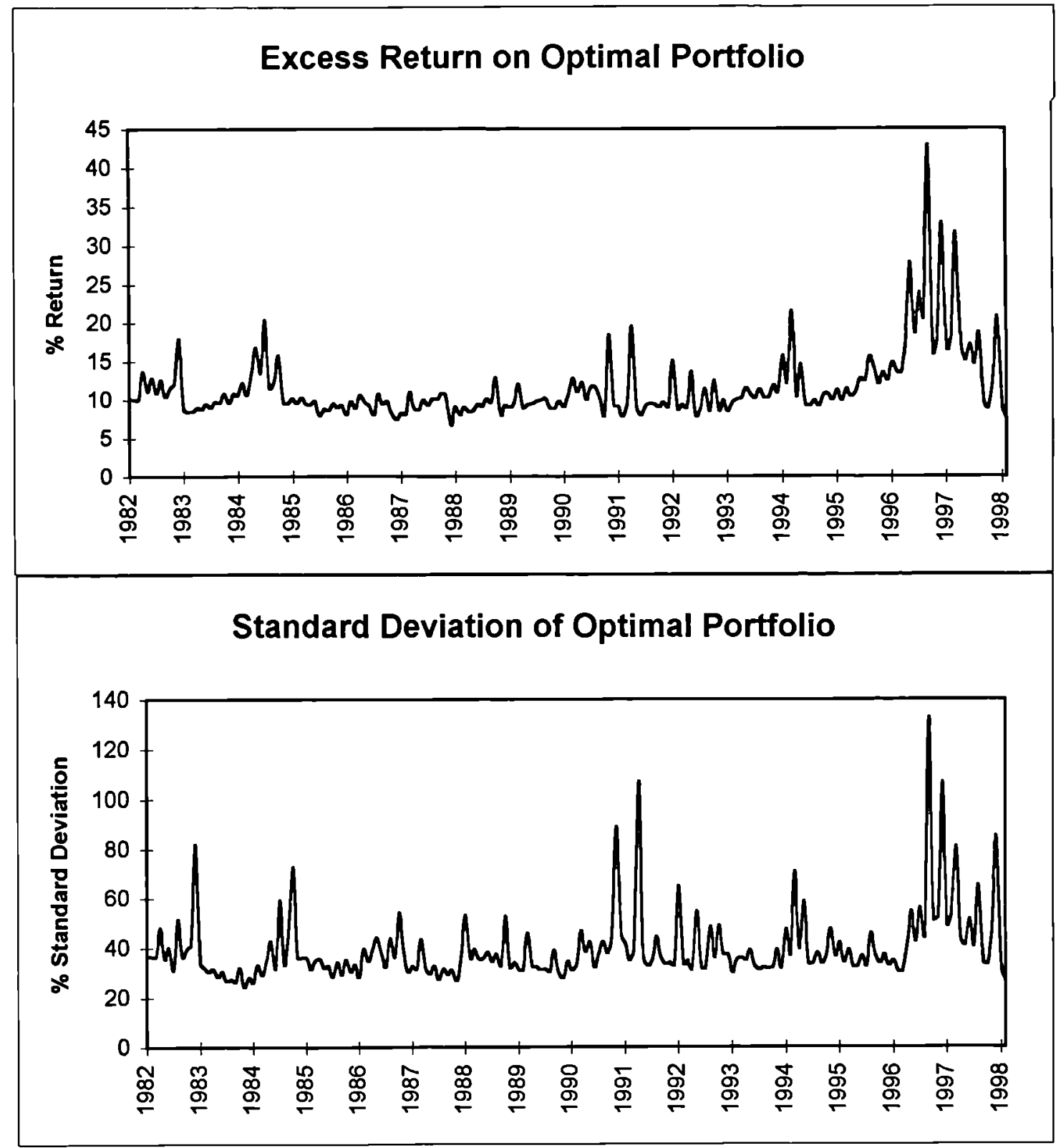




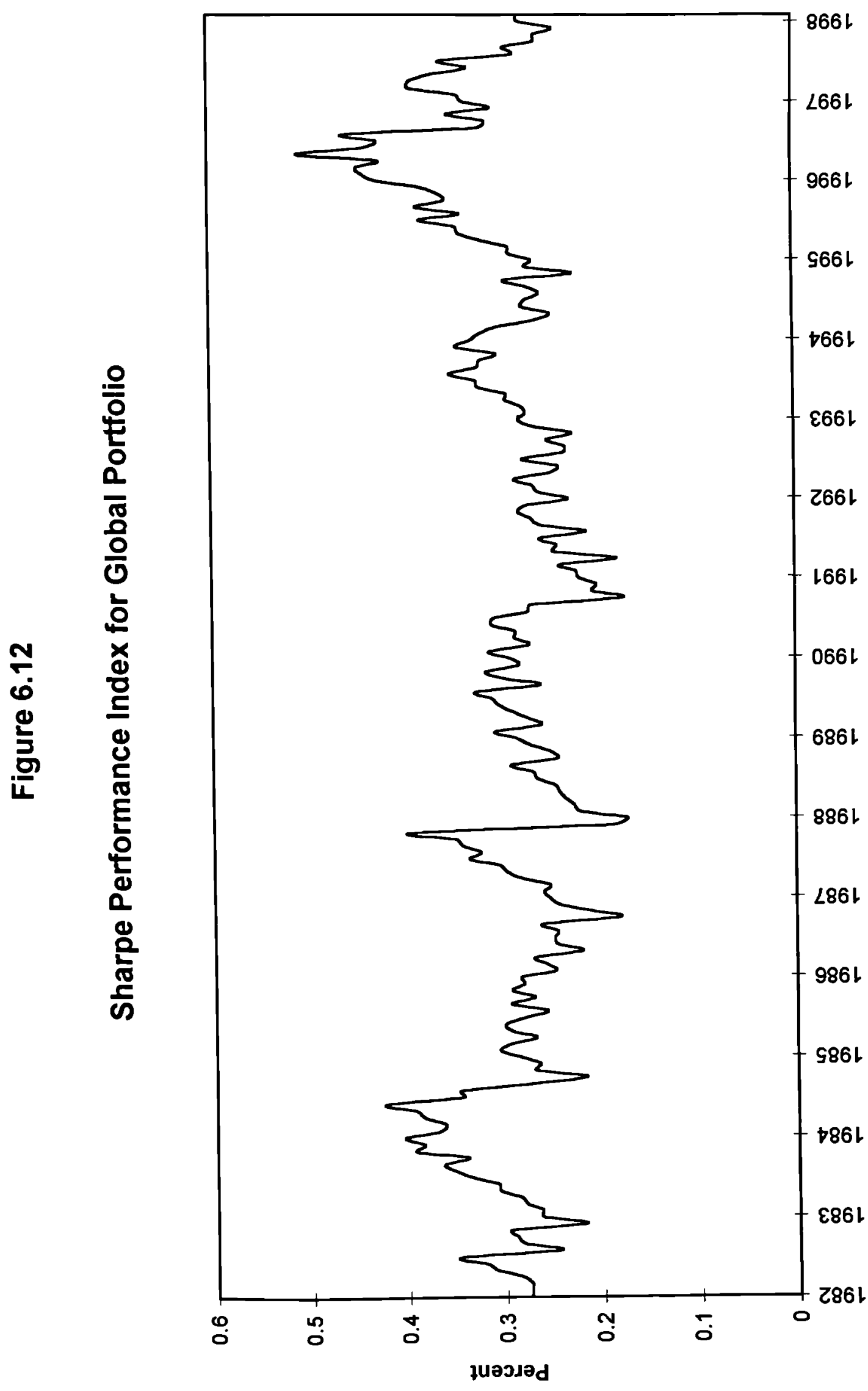

$\stackrel{\infty}{\circ}$ 

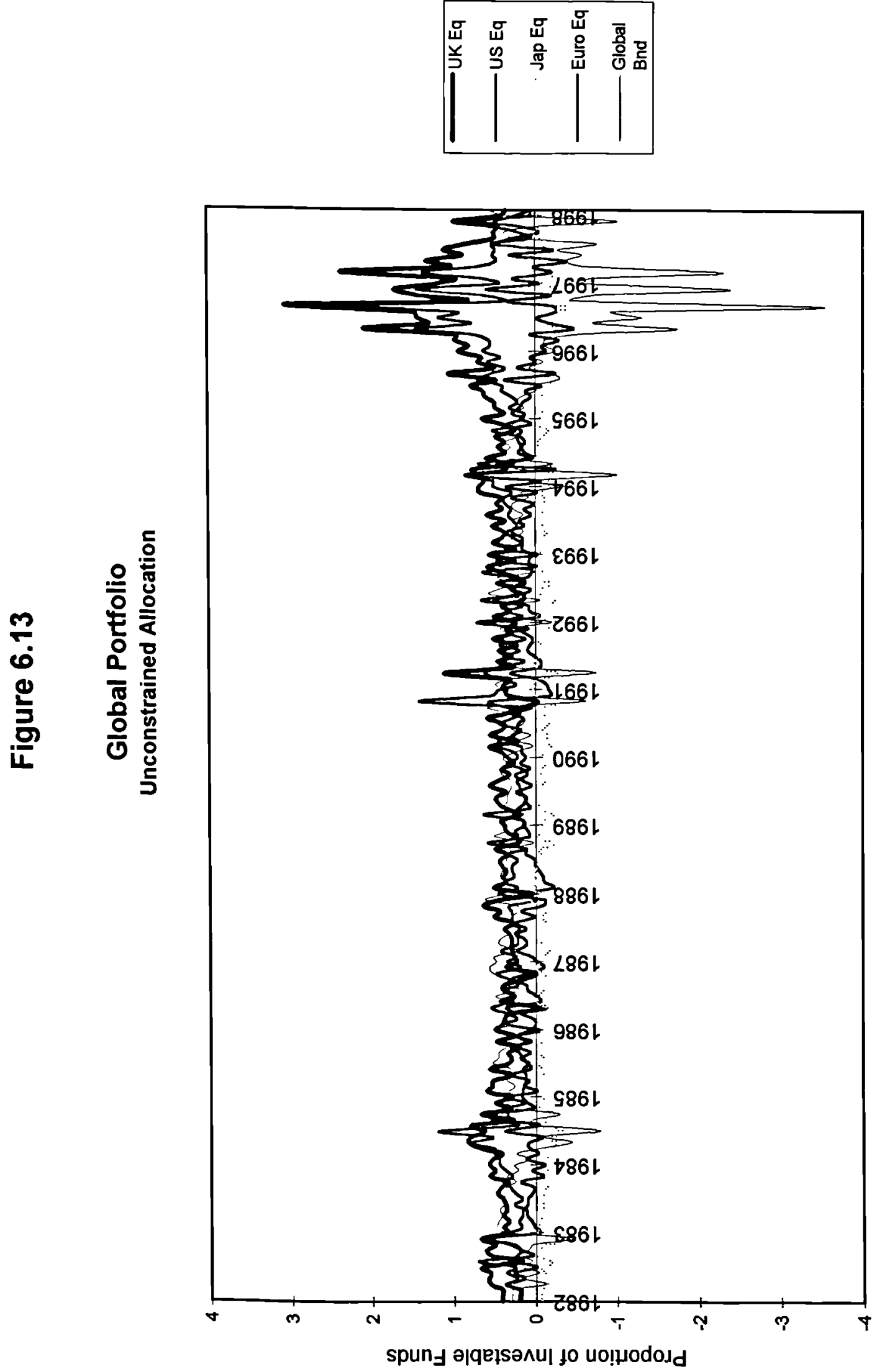

$\infty$ 


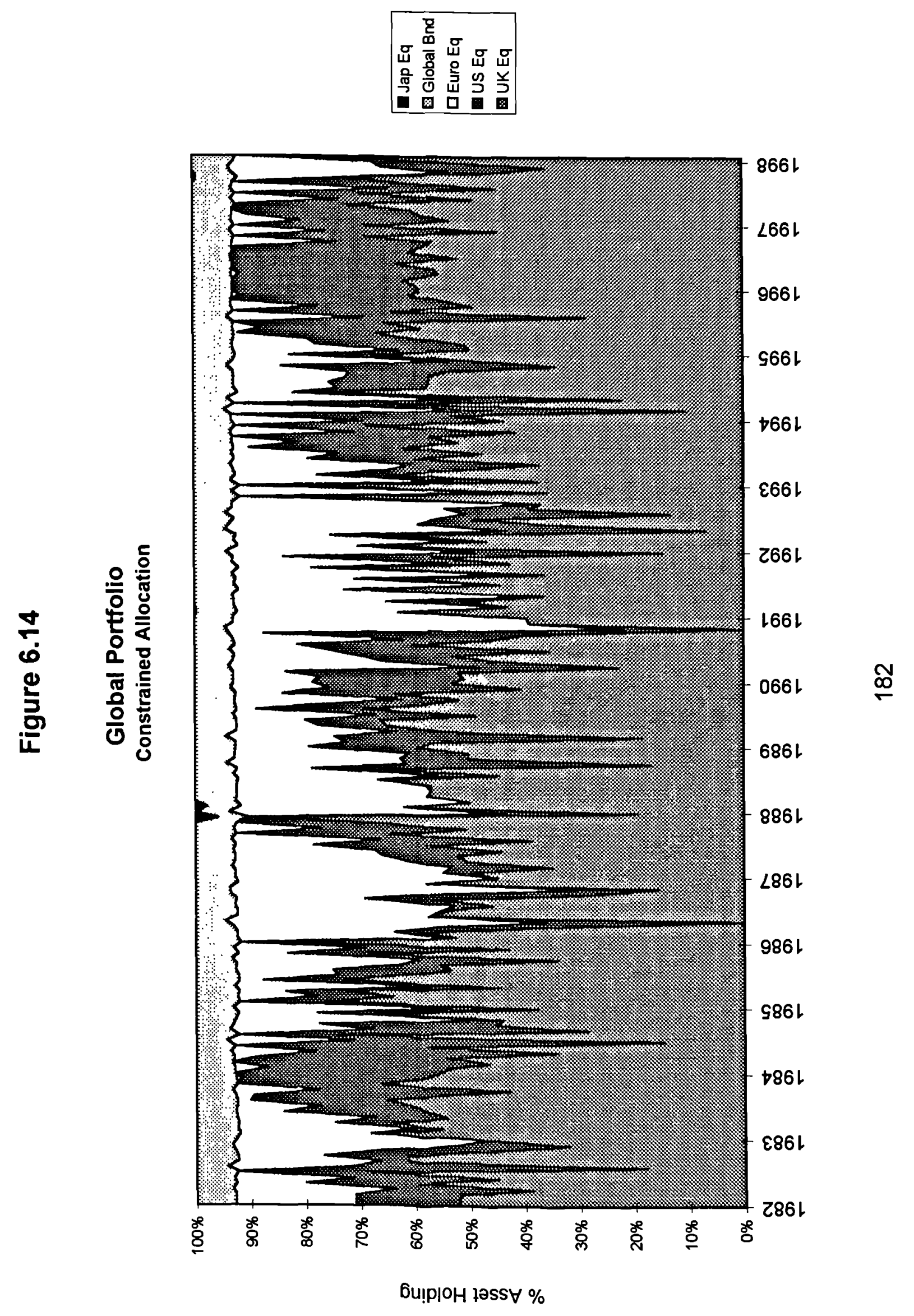




\section{Chapter 7}

\section{Macroeconomic Influences on}

\section{the Optimal Allocation of}

\section{Domestic UK Assets}

\subsection{Introduction}

This chapter seeks to build bridges between observed macroeconomic phenomena and the world of financial markets by investigating the relationship between financial asset returns and macroeconomic variables. This is motivated by the fact that stock market volatility appears to be related to the general well-being of the economy. Historically, it has been noted that financial market turbulence has been greatest in times of recession (see 
Schwert(1989)). We propose to exploit the predictability of future financial asset price movements using information contained in macroeconomic data. This should give us a more accurate estimate of the conditional risk associated with each asset and subsequently produce better performing portfolios of risky assets. Clarke and De Silva(1998) note that "state-dependent variation in asset returns has strong implications for identifying an optimal asset allocation strategy". Klemkosky and Bharati(1995) employ an ad hoc model of asset returns to show that short-term predictability can be used to build profitable asset allocation models.

We extend the analysis of chapter 4 where it was seen that UK investors investing in domestic assets only enjoyed significant reductions in the riskiness of their portfolios by employing time-varying conditional covariance matrices as measures of risk as opposed to more traditional unconditional estimates. Continuously re-balancing the portfolio in response to changes in the conditional covariance matrix improved portfolio performance. Here, we again use our multivariate $\operatorname{GARCH}(1,1)$ model to capture time variation in the conditional second order moments of financial asset returns. However, by jointly modelling the financial asset returns and the macroeconomic variables, we can immediately assess the influence of these variables on both the conditional mean and conditional second order moments of the financial assets. As in chapter 4, we take the perspective of an investor who may 
allocate wealth among domestic UK assets. In our analysis, we have three domestic risky assets: equity, a long-term government bond and a shortterm government bond. We choose inflation as our macroeconomic variable and allow it to exert an influence on the asset allocation decision through its covariance with each of the financial assets. We find that the change in domestic inflation can wield a strong influence on the processes governing the behaviour of the returns of UK financial assets. This is especially true in the conditional second order moments.

Taking the influence of inflation into account has a significant impact on the proportions in which the risky assets are held in the optimal portfolio period by period. For the case of the portfolio whose weights are restricted to be non-negative, the mean asset holdings over the 20 year sample are significantly changed. In particular, the percentage of equities increases from $70 \%$ to $74 \%$ while the relative dominance of the long bond over its short-term counterpart is greatly reduced with the short bond increasing its share of the portfolio from $10 \%$ to $12 \%$ while the proportion of long bonds in the portfolio slips from $20 \%$ to $14 \%$. Therefore this effect is important and deserves to be taken into account.

The remainder of the chapter is structured as follows: section 7.2 presents a brief overview of the existing literature on the relationship between macroeconomic variables and returns on financial assets. It also pays particular 
attention to the relationship between inflation and stock returns which has become an empirical puzzle. Section 7.3 presents the econometric model, discusses some refinements that are important in this implementation of the model and finally describes the data used in this application. The results of the analysis are presented in section 7.4 while section 7.5 contains our concluding remarks.

\subsection{Asset Returns and Macroeconomics}

\subsubsection{The Empirical Evidence}

From the mid-80's, there has been a growing literature on the predictability of financial asset returns. Generally, these studies focus on macroeconomic and financial variables in an effort to exploit any information that can be extracted to aid the prediction of movements in future financial asset prices. This literature reflects the importance attached to macroeconomic data by financial market participants. While we focus mainly on the predictability of stock returns in this section, similar factors can be thought to affect bond markets. In this respect, we rely on Fama and French(1989) who provide evidence that forecasts of excess bond and stock returns are correlated, and Campbell and Ammer(1993) who find that variables which are useful in forecasting excess stock returns can also be used to forecast excess bond 
returns.

Roll and Ross(1986) are credited with being the pioneers in the asset return predictability literature following their paper identifying factors that can potentially be used to predict US stockmarket prices. This paper found that the spread between long-term and short-term interest rates (a measure of the term structure or slope of the yield curve), expected and unexpected inflation, industrial production, and the spread between high- and low-grade bonds are all significantly priced in the US stockmarket. More recently, Jankus(1997) shows that expected inflation is also a useful predictor of future bond yields.

In a similar vein, many papers provide evidence of the explanatory power of the dividend yield over annual US stock returns (Rozeff(1984), Campbell and Shiller(1988), Hodrick(1992), Patelis(1997) etc.). A positive correlation between the term structure of interest rates and price movements in stockmarkets has been documented for the US by Keim and Stambaugh(1986), Campbell(1987) and Patelis(1997) while the slope of the term structure is found to have forecasting power for excess bond returns in Campbell and Shiller(1991) and Fama(1984). Using cross-sectional data, Fama and French(1992,1995) find support for a negative relation between the Price/Book ratio and US stock returns. Meanwhile, Peseran and Timmermann(1995) identify a wide range of factors that can influence returns on 
US equities. These factors include the earnings-price ratio, the rate on the one- and twelve-months government Treasury bills, the change in domestic inflation, the change in industrial production and monetary growth.

A parallel literature has emerged in the UK. A large number of studies show that many macroeconomic variables and financial ratios are also significantly priced in the UK stockmarket. Clare, Thomas and Wickens(1993) show that the Gilt to Equity Yield Ratio(GEYR), i.e. the ratio of the yield on long government bonds to the dividend yield, has explanatory power in predicting UK stock price movements.

Clare and Thomas(1994) identify a whole host of factors that were found to have significant predictive power over returns on UK stocks. They found that the current account balance; US equity markets; German Equity markets; UK 90-day Treasury bill rates; the differential between UK and US 90-day Treasury bill rates; the irredeemable government bond index; the corporate bond index; the term structure of interest rates; and the dollar to pound exchange rate could all be used to forecast future price movements in the UK stockmarket. Clare, Smith and Thomas(1996) focus on the ability of lagged own values and lagged returns on other markets to predict UK stock returns.

More generally, Asprem(1989) conducted a wide ranging analysis looking at the relationship between stock market indices, portfolios of assets and 
macroeconomic phenomena in ten European countries. Interestingly for our analysis, he finds that the linkages between stock prices and macroeconomic variables are most pronounced in Germany, the Netherlands, Switzerland and the UK. Asprem begins by investigating the relationship between stock prices and measures of real economic activity. He finds that expectations about future changes in industrial production are positively related to the stock price while lagged values of employment have an inverse relationship with the stock index. However, it should be pointed out that many of the reported coefficients are not statistically different from zero and for the UK, the employment-stock price relationship is not investigated due to lack of data. Therefore it seems that at best, these links are tenuous and current or lagged values of real economic activity variables contain little predictive power over future asset returns.

The next category of macroeconomic variable analysed is the exchange rate and in particular, the change in the effective trade-weighted exchange rate for each country. Once more, there is no evidence of a statistically significant relationship between stock prices and either current or lagged values of the exchange rate variable.

Then Asprem focuses on consumption. Only for the UK is there evidence of a significant negative relation between stock prices and consumption. This result offers support for the consumption based CAPM of Bree- 
den(1979) which predicts that asset returns and the marginal propensity to consume should be negatively correlated. However, when the change in imports is included as a proxy for consumption, the results are highly supportive of a negative correlation between the variables, especially in the more developed financial markets of Germany, France and the UK. The better performance of the imports variable is probably due to the fact that it is a more volatile series than consumption.

Turning his attention to the interest rate, Asprem again finds strong evidence of a negative relationship between stock prices and current and lagged values of the interest rate. The most conclusive results are obtained for Germany, the Netherlands, Switzerland and the UK. It should be noted that the study uses long-term interest rates though it is more usual for financial market participants to measure opportunity costs by the short interest rate. Furthermore, Asprem shows that the current value of the US term structure of interest rates has significant explanatory power over stock returns in the aforementioned subset of countries. We have already seen that this relationship holds for the US.

Finally, Asprem looks at the potential relationship between stock prices and inflation and money supply effects. Consistent with other studies, he finds that asset prices and inflation are negatively correlated. This relationship appears to hold both for lagged values of the change in inflation 
as well as expected future changes in the variable. Since money supply and inflation are assumed to be positively linked through the quantity theory of money, we would expect a similar relationship between money supply and stock prices. Using the monetary base, M0, as a measure of money supply, results are supportive of a negative correlation, but only in the UK is the relationship statistically significant.

Despite this wealth of evidence on the predictability of the mean process of asset returns, the ability of macroeconomic variables to influence higher order moments has been afforded little attention in the literature. In a test of the international CAPM, Engel and Rodrigues(1989) allow macroeconomic variables to influence the variance process of an ARCH model. The variables are chosen in an ad-hoc manner and introduced into the model one at a time. Their findings are that the square of the unanticipated monthly growth rate of dollar oil prices and the monthly growth rate of the US M1 are significant explanatory variables of the variance of residuals. In a more recent study, Clare, O'Brien, Thomas and Wickens(1998) demonstrate that when a number of macroeconomic variables are subjected to simultaneous shocks, they can have a very significant influence on the conditional variance-covariance matrix of asset excess returns. In this study, up to four macro shocks are introduced simultaneously but it is found that the improvement in the performance of the model over the one macro factor 
specification is not large.

In summary, there is strong evidence for both US and Europe that a broad range of macroeconomic factors are significantly priced in their stock markets. The general findings across markets are that changes in inflation (both realised and expected), interest rates and imports (or consumption) have a negative correlation with returns on stock indices. In contrast, yield curve measures (term structure of interest rates) tend to have a positive relationship with movements in stock prices. However, there is little evidence available on the ability of macroeconomic variable effects on the variances and covariances of financial assets. This issue is a major part of our focus since these second order moments are crucial in the optimal portfolio selection procedure.

\subsubsection{Stock Returns and Inflation}

In our empirical analysis, we use inflation as our macroeconomic variable and look at its effects on both the mean and second order moments of financial asset returns. Inflation is chosen for a number of reasons. Firstly, the empirical evidence presented above is supportive of a strong relationship between inflation and asset returns. This motivates the inclusion of an inflation variable in the mean equation. Secondly, since we are working with nominal asset returns, it has been argued by Schwert(1989) that if the 
inflation of goods' prices is uncertain, then the volatility of nominal asset returns should reflect inflation volatility. This argument provides a good basis for expecting a strong relationship between the second order moments of the inflation variable and of financial asset returns. Thirdly, we would like to investigate if the puzzling relationship between stock returns and inflation holds when higher order moments are taken into account. Theory suggests that the relationship should be both positive and 1:1. This expected relationship stems from the fact that an increase in inflation raises the value of real assets and since stocks are believed to be a claim on real assets, it is reasonable to expect a positive 1:1 relationship. Therefore, stocks should prove to be an effective hedge against inflation ex post. Likewise, based on an application of the Fisher hypothesis to stocks, we would expect an equivalent relationship ex-ante. Conversely, the empirical evidence finds that the relationship is consistently negative across countries and over different time periods (see Bodie(1976) and Fama and Schwert(1977) for the US and Solnik(1983) and Gultekin(1983) for a number of other countries).

The search for an explanation to this puzzling phenomenon has attracted a great deal of attention over the past two decades. Firstly, both Nelson(1976) and Fama(1981) offered an explanation based on the theory of money demand. They argued that since it was clear from money demand theory that there should be a negative relationship between inflation and 
the growth of real activity and since stock returns were a good predictor of real activity, then this gave rise to a negative relationship between stock returns and inflation. Fama provided empirical evidence of the two former relationships and argued that this induced the third.

Geske and Roll(1983) extend the arguments of Nelson and Fama. They posited that the causality runs from stock returns to inflation and not viceversa. They argue that the negative relationship results from a number of fiscal and monetary reactions to an adverse economic shock, with the stock return providing a signal of this shock. In particular, an adverse shock to the economy will reduce the stock return as well as increasing unemployment and decreasing corporate earnings. Consequently, the tax revenue accruing to the government will be reduced, causing an increase in their deficit. Part of this deficit may be financed by issuing more bonds and some of these may be bought by the government, causing an increase in the monetary base and subsequently in inflation.

Another, more succinct, explanation of the puzzle has been offered by Groenewold, O'Rourke and Thomas(1997) in the context of a small systematic macroeconomic model. They find that the negative sign emerges from the macroeconomic interactions of inflation with a wide range of other macro variables. Some of these other factors affect stock return as well as any direct inflation effect e.g. a rise in expected inflation increases real 
output and this impacts negatively on stock returns.

\subsection{Empirical Model and Data}

\subsubsection{Model}

We employ our multivariate $\operatorname{GARCH}(1,1)$ model to estimate the time varying covariance matrices of asset excess returns upon which the asset allocation decision depends. As before, we adopt the parameterisation of the model as set out in chapter 3 . However, in this application we jointly model the financial asset returns and the macroeconomic variables by including both in the vector of dependent variables in the conditional mean equation. Therefore, the macro factors influence the conditional mean equation. These factors also exert an influence on the variances and covariances of the financial assets through their interactions in the M-GARCH process. The model is specified as follows:

$$
\begin{aligned}
\mathbf{z}_{t} & =\boldsymbol{\alpha}+\boldsymbol{\beta} \mathbf{z}_{t-1}+\boldsymbol{\gamma d u m} 87+\boldsymbol{\xi}_{t} \\
\boldsymbol{\xi}_{t} & \mid \Psi_{t-1} \sim N\left(0, \mathbf{H}_{t}\right) \\
\mathbf{H}_{t} & =\mathbf{V}^{\prime} \mathbf{V}+\mathbf{A}^{\prime}\left(\mathbf{H}_{t-1}-\mathbf{V}^{\prime} \mathbf{V}\right) \mathbf{A}+\mathbf{B}^{\prime}\left(\boldsymbol{\xi}_{t-1} \boldsymbol{\xi}_{t-1}^{\prime}-\mathbf{V}^{\prime} \mathbf{V}\right) \mathbf{B}
\end{aligned}
$$


where $\mathbf{z}=(\mathbf{r}, \mathbf{m})^{\prime}$ and $\mathbf{r}=(u k e q, l b d, s b d)^{\prime}$ is a $3 x 1$ vector of excess returns on $\mathrm{UK}$ financial assets with $u k e q, l b d$ and $s b d$ representing $\mathrm{UK}$ equity, long government bonds and short government bonds respectively. $\mathbf{m}$ is a $1 x 1$ vector of macroeconomic factors which, in this analysis, is the change in the domestic inflation rate.

However when we include macroeconomic variables, the speed which they exert their influence becomes very important. In this respect, the manner in which we define the $\mathbf{V}, \mathbf{A}$ and $\mathbf{B}$ matrices in the second order moment equation is vital. A simple example will illustrate the timing difference under two alternative definitions of the aforementioned matrices. Let us assume that we have a $2 x 1$ vector of dependent variables with elements $r_{t}$ and $m_{t} \cdot r_{t}$, represents a financial asset and whose conditional variance is denoted by $H_{11}$ and $m_{t}$, is a macroeconomic factor with conditional variance $H_{22}$. The covariance between the two variables is given by $H_{12}$.

Definition 1. V, A and B are symmetric, lower triangular matrices.

Then

$$
H_{11, t}=V_{11}^{2}+A_{11}^{2} H_{11, t-1}+B_{11}^{2} \varepsilon_{1, t-1}^{2}
$$

and

$$
\varepsilon_{1, t-1}=r_{t-1}-\alpha_{1}-\beta r_{t-2}-\delta m_{t-2}
$$

Therefore, we can see that when we define the matrices in this way, it 
takes two periods for the macroeconomic variable to influence the conditional second order moments of the process.

Deflnition 2. V, A and B are full, symmetric matrices.

Then

$$
\begin{aligned}
H_{11, t}= & \left(V_{11}^{2}+V_{12}^{2}\right)+ \\
& \left(A_{11}^{2} H_{11, t-1}+2 A_{11} A_{12} H_{12, t-1}+A_{12}^{2} H_{22, t-1}\right)+ \\
& \left(B_{11}^{2} \varepsilon_{1, t-1}^{2}+2 B_{11} B_{12} \varepsilon_{1, t-1} \varepsilon_{2, t-1}+B_{12}^{2} \varepsilon_{2, t-1}^{2}\right)
\end{aligned}
$$

From equation (7.4), it is obvious that the macroeconomic variable can now exert its influence on the conditional variance of the financial asset with only a one period time lag through both its own variance, $H_{22, t-1}$ and the covariance term, $H_{12, t-1}$. This specifiation of the matrices in the conditional second order moments of the process is clearly preferable as we expect financial markets to react to new information with minimum delay.

Based on the above argument, we estimate the model as specified in equation (7.1) with V, A and B defined as full, symmetric matrices.

\subsubsection{Data}

The data on the financial assets used in this chapter is the same as in chapter

4. Once more, equity is represented by the Financial Times All Share Index; 
long UK government bonds represented by the FT British government stock over 15 years index; and short government bonds represented by the FT British government stock under 5 years index. The data used in this chapter is annualised monthly total returns for each asset and all are expressed in excess of the riskless rate. The riskless rate of return is proxied by the rate on UK government 30-day Treasury bills. The inflation rate is calculated from the UK Retail Price Index. However, econometric tests provided evidence that the inflation rate contained a unit root and consequently we choose to work with the first difference series to ensure that all variables in the analysis are stationary. The data covers the sample period from January 1976 and February 1997. Again, all data was sourced from DATASTREAM.

\subsection{Estimation Results}

The model specified in the previous section by equation 7.1 was maximised subject to the $\mathrm{BHHH}$ algorithm. The maximisation routine produced the results reported below in sections 7.4.1 and 7.4.2. The numbers appearing in parentheses are $t$-statistics. Since $\mathbf{V}, \mathbf{A}$ and $\mathbf{B}$ are symmetric, we report only the lower triangle. 


\subsubsection{Mean Matrices}

$$
\boldsymbol{\alpha}=\left[\begin{array}{c}
11.93 \\
(3.35) \\
4.76 \\
(1.56) \\
0.94 \\
(0.97) \\
-0.032 \\
(-0.83)
\end{array}\right], \boldsymbol{\beta}=\left[\begin{array}{cccc}
-0.04 & 0.24 & -0.33 & -5.13 \\
(-0.65) & (1.77) & (-0.92) & (-0.98) \\
0.03 & 0.02 & 0.14 & -4.27 \\
(0.52) & (0.19) & (0.56) & (-0.98) \\
-0.04 & 0.06 & 0.04 & -0.01 \\
(-1.67) & (1.55) & (0.54) & (0.1) \\
0.001 & -0.001 & 0.004 & 0.402 \\
(0.72) & (-0.44) & (-0.59) & (4.62)
\end{array}\right], \boldsymbol{\gamma}=\left[\begin{array}{c}
-420.98 \\
(-1.43) \\
0 \\
0 \\
0
\end{array}\right]
$$

\subsubsection{Covariance Matrices}

$$
\mathbf{V}=\left[\begin{array}{ccccc}
58.16 & & & \\
(24.10) & & & \\
24.38 & 34.88 & & \\
(10.22) & (34.29) & & \\
9.01 & 8.47 & 8.69 & \\
(10.35) & (13.31) & (15.27) & \\
-0.104 & -0.033 & -0.046 & 0.536 \\
(1.83) & (0.56) & (0.48) & (5.11)
\end{array}\right]
$$


$\mathbf{A}=\left[\begin{array}{cccc}0.08 & & & \\ (0.25) & & & \\ -0.51 & -0.003 & \\ (3.72) & (0.01) & \\ -0.17 & 0.58 & 0.27 & \\ (0.86) & (4.06) & (0.92) & \\ 0.06 & -0.15 & -0.08 & -0.48 \\ (1.39) & (3.73) & (1.43) & (1.51)\end{array}\right], \mathbf{B}=\left[\begin{array}{ccccc}0.17 & & & \\ (3.78) & & & \\ 0.02 & 0.015 & \\ (0.59) & (0.45) & & \\ 0.087 & -0.013 & 0.21 & \\ (5.25) & (0.41) & (2.32) & \\ -0.003 & 0.006 & 0.004 & -0.19 \\ (1.46) & (2.44) & (0.42) & (1.44)\end{array}\right]$

\subsubsection{Discussion of Results}

The results suggest that the level of the excess returns on risky UK financial assets are largely unpredictable in the mean equation. Only the long government bond exerts any influence on UK equity, which is consistent with the Thomas and Wickens(1993) finding that the GEYR variable has forecasting power over future UK equity returns. Both long-term and shortterm government bonds are largely unpredictable. The change in domestic inflation is statistically insignificant in predicting the first order moments of UK financial assets. Despite the imprecise estimation of these parameters, it is noteworthy that these parameters are negative for all assets and large for both stocks and long government bonds. This is consistent with 
the empirical studies reported above and therefore explicitly modelling the second order moments has not resolved the puzzle.

When we look at the second order moments, a more important role for the macroeconomic variable emerges. The inflation variable now plays a major part in the determination of the conditional variances and covariances of the system. In the long run, the change in inflation exerts a significant impact on the variability of $U K$ equity returns, see $V_{41}$, but its influence on the volatility of either government bond is not significantly different from zero. The long run conditional covariance matrix resulting from the estimation is:

$$
\mathbf{V}^{\prime} \mathbf{V}=\left[\begin{array}{lllll}
3382.44 & & & \\
1417.91 & 1810.78 & & \\
524.21 & 515.14 & 228.43 & \\
-6.03 & -3.69 & -1.61 & 0.30
\end{array}\right] \text {, }
$$

and it is interesting that the largest negative correlation is -0.59 for equity returns and the inflation variable.

The negative sign on the covariances between the financial assets and the change in inflation is again consistent with the Groenewold et al.(1997) story of the negative correlations resulting from interactions in the wider macroeconomic world, with inflation impacting on the financial sector in- 
directly through other macro factors. Of course, these negative covariances will tend to reduce the overall conditional volatilities of the individual assets. Therefore, the negative signs in the mean equation are to be expected since lower volatility will require a lower excess return to induce the investor to hold the asset.

The short-run matrices, $\mathbf{A}$ and $\mathbf{B}$, have many insignificant parameters but enough are significant to justify the approach adopted. The off-diagonal terms $A_{21}, A_{32}$ and $B_{31}$ all suggest that short-term revisions to the composition of the portfolio will be required to achieve optimality. Focusing on the effects of the macroeconomic variable, we find that the change in domestic inflation is a very important determinant the long government bond volatility, with both $B_{42}$ and $A_{42}$ statistically significant. However, its short-run influence on both equity and short bond return volatility is less significant with parameters $A_{41}, B_{41}$ and $A_{43}$ at best marginally significant.

There is sufficient support for the inclusion of this variable in the determination process of the time-varying conditional variances and covariances of UK financial assets which will be used to locate and identify the optimal portfolios of risky assets period by period. The lagged change in domestic inflation contains important information which may be exploited by financial market participants to predict future asset volatility and hence have more accurate inputs into the asset allocation decision. Figure 7-1 depicts 
the total conditional variances of the assets and their long-run values. From this we appreciate the importance of the short-run deviations. Taking these short-run deviations into account allows us to develop a tactical asset allocation strategy that reflects changes in both the perceived riskiness of assets and macroeconomic conditions.

\subsubsection{Frontier Movements}

Having derived the time varying variance-covariance matrices, we then proceed to partition the matrices to extract the elements relating to the financial assets. Following the estimation process, the effects of the macroeconomic variables have already impacted on these elements (see equation (7.4)). Using the estimated conditional variances and covariances and historical returns as a proxy for expected returns ${ }^{1}$, we can first generate the time-varying portfolio frontiers. These frontiers provide strong support in adopting this approach as they provide evidence of significant time variation in both the shape and location of the frontier. Figure 7-2 shows the distribution of the frontiers generated by this model. It plots the mean, minimum and maximum frontiers for the entire 20 year sample. It reveals that the distribution is highly positively skewed with a long tail to the right. This will be important as assuming a static covariance matrix would have

\footnotetext{
${ }^{1}$ The reason for using historical returns as a proxy for expected returns is discussed in Chapter 4.
} 
greatly underestimated the risk during periods in this tail. At a glance, we can see that the range of movement is quite large. Focusing on the minimum variance portfolios, we have a minimum standard deviation of $6 \%$ and a maximum of $22 \%$. Clearly, the shape of these frontiers change also.

Contrasting this distribution with its counterpart generated without taking any macroeconomic factors into account also provides us with some interesting evidence. Figure 7-3 contrasts these frontier distributions. The heavy line depicts the frontier generated following the inclusion of the domestic inflation variable. In general, this distribution is shifted to the left, which helps the investor achieve a higher expected return for any given level of risk. Admittedly, there is considerable overlap in the distributions and for both the mean and minimum frontiers the efficient segments are close and converging. However, for an investor concerned with holding the minimum-variance portfolio, the explicit modelling of the inflation variable produces portfolios which deliver a substantial reduction in risk.

Figure 7-4 tells a similar story. This graph contrasts the use of conditional and unconditional estimates of the covariance matrix. It depicts the mean time-varying frontier and the frontiers generated by both the longrun estimate $\left(\mathbf{V}^{\prime} \mathbf{V}\right)$ and a simple OLS estimate of the unconditional matrix of asset excess returns. The most striking feature is that the conditional frontier lies closer to the origin than either of the two unconditional fron- 
tiers. It is therefore possible for investors to reduce their portfolio risk by re-balancing their portfolios each period. Another implication is that the actual portfolio risk borne by investors who use the long-run covariance matrix will be different from that shown by the long-run frontier.

\subsubsection{Optimal Portfolios}

Having generated the portfolio frontier for every period of the analysis, we can identify the location of the optimal portfolio of risky assets. Figure 75 shows how the excess return and the standard deviation of this portfolio behaved over the sample. We then calculate the Sharpe Performance Index, $S P I_{t}=\frac{\text { return }}{\text { risk }_{t}}$, for the optimal portfolios of this model and the corresponding model without macroeconomic effects. Figure 7-6 plots the SPI values. The two models produce optimal portfolios with similar SPI values and actually both have means of 0.16 . This is consistent with the observation that the efficient segments of the frontiers were close and converging. Despite a similar portfolio performance, the constituents of the portfolio can still differ and its likely that with the inflation effects, the composition of the optimal portfolios will change.

Once we identify the location of the portfolio, we solve for the proportions in which the risky assets should be held in order for the investor to hold the optimal portfolio of risky assets. Figure 7-7 shows this allocation 
over the entire sample. We notice that taking the inflation effects into account leads to larger portfolio revisions and hence increased volatility in the holding of the risky assets. Table 7.1 summarises the key features of this allocation where the investor is allowed to sell short the assets without limit.

\begin{tabular}{|l|l|l|l|}
\hline & Mean & Min. & Max. \\
\hline Equity & $73 \%$ & $33 \%$ & $317 \%$ \\
\hline Long Bond & $16 \%$ & $-16 \%$ & $207 \%$ \\
\hline Short Bond & $11 \%$ & $-425 \%$ & $83 \%$ \\
\hline
\end{tabular}

Table 7.1: Summary statistics for unrestricted portfolio with Macro influences

UK equities dominate the optimal domestic portfolio of risky assets. On average, equity accounts for $73 \%$ of the portfolio and is never held short. The holding of UK stocks is quite often in excess of $100 \%$ of the investor's wealth with one or other of the bonds being held short to make this investment possible. Also in this analysis, we notice that the holdings of the domestic government bonds are much more volatile following the inclusion of the macroeconomic factor. Now, the long bond has a mean holding of $16 \%$ reduced from $20 \%$. The investor also holds this asset short on many more occasions than in the earlier study reported in Chapter 4 . This may be explained by the fact that the inflation variable exerted its largest and most significant short-run influence on the volatility of this asset. Hence the 
holding of the asset is much more volatile. As before, the short government bond is the most volatile asset, ranging from $-425 \%$ to $83 \%$ of investor wealth. However, when we focus on the mean holding of the asset over the period, we observe that its importance within the portfolio has remained largely unaltered with only a slight increase in its mean value, from $10 \%$ up to $11 \%$. This would seem to result from a smaller inflation influence on its conditional volatility. This may be plausible due to the fact that inflation may be more predictable over shorter horizons and hence not be as great a worry for the investor in the short-term asset.

As in our previous studies, the holdings of the financial assets under this investment strategy are deemed to be excessively volatile to provide a viable method of tactical asset allocation. Consequently, we decide to re-allocate the assets using an alternative allocation strategy where short sales are prohibited since many investors are constrained, either by law or financially, from holding negative positions in any asset class. Again, the target rate of return on this portfolio of risky assets is the mean rate of return on the unrestricted portfolio of assets.

This method of allocation leads to portfolios of assets that are relatively stable, as can be seen from figure 7-8. The relative importance of the assets remains largely unchanged. Equities account for the major part of the portfolio with an average position of $74 \%$ of the portfolio. The long bond 
continues to dominate the short government bond. However, this dominance is not as pronounced as in the unrestricted allocation. The mean holdings of the long and short bonds are $14 \%$ and $12 \%$ of the portfolio respectively. However the range within which the holdings of the various assets move is greatly dampened with the variability of the equity, long bond and short bond holdings reduced by factors of approximately 28,8 and 31 respectively. Table 7.2 summarises the asset holdings under this investment strategy.

\begin{tabular}{|l|l|l|l|}
\hline & Mean & Min. & Max. \\
\hline Equity & $74 \%$ & $66 \%$ & $76 \%$ \\
\hline Long Bond & $14 \%$ & $8 \%$ & $34 \%$ \\
\hline Short Bond & $12 \%$ & $0 \%$ & $16 \%$ \\
\hline
\end{tabular}

Table 7.2: Summary statistics for restricted portfolio with Macro influences

When we contrast these asset weights to those resulting from the analysis without taking the effect of domestic inflation into account, we see that both the UK equity and short-term government bond increase their positions within the portfolio, while these increases are offset by a reduction in the holding of the long bond. The changes in the perceived riskiness of the assets induces the investor to hold these new asset proportions. Table 7.3 summarises the mean asset holdings of each asset in the restricted optimal portfolio generated by the models with and without the inflation effect. 


\begin{tabular}{|c|c|c|c|}
\hline & No Macro factor & With Macro factor & Change \\
\hline Equity & $70 \%$ & $74 \%$ & $+4 \%$ \\
\hline Long Bond & $20 \%$ & $14 \%$ & $-6 \%$ \\
\hline Short Bond & $10 \%$ & $12 \%$ & $+2 \%$ \\
\hline
\end{tabular}

Table 7.3: Macroenomic effects on mean asset holdings

It should be noted that the relative importance of each asset class in the portfolio is unchanged. UK equity continues to be the major constituent of the optimal portfolio and the long government bond retains its dominance over its short-term counterpart. However, equity gains most with an increase of $4 \%$, while the short government bond increases its mean position by $2 \%$. The long bond suffers a decline in its attractiveness as a investment instrument and its average position in the portfolio falls by $6 \%$. It is interesting that the assets with the largest negative correlation with the inflation series become more attractive investment vehicles.

\subsection{Conclusion}

The aim of this chapter was to evaluate the importance of macroeconomic variable effects on the optimal asset allocation decision of a UK investor who wishes to tactically allocate his funds for investment in risky assets among three major classes of UK financial assets. This analysis extends the work of chapter 4 where we addressed the portfolio selection problem in the absence of any influences from the wider economic environment. The 
incorporation of macroeconomic variables into the allocation strategy is a response to the growing literature on the predictability of financial asset returns. This analysis could also be motivated by the observation that many financial market participants place great emphasis on having up to date information on a wide range of variables in an effort to exploit any information that would enable them to make abnormal profits.

Our multivariate GARCH $(1,1)$ model provides a neat way to incorporate macroeconomic effects into the portfolio selection decision as we can jointly model the financial asset returns and the macroeconomic variables. This allows the variable to exert an influence on both the first and second order moments of the process and hence on the asset allocation through its covariance with each of the financial assets. We discover that each of the parameter matrices in the second order moments should be specified as full symmetric matrices in order to minimise the time lag with which the macro variable exerts its influence on the variances and covariances of the financial assets. We choose inflation as our macroeconomic variable in this analysis.

We find that the change in inflation fails to forecact future asset excess returns but that the conditional second order moments are much more predictable. The inflation variable has a significant impact on the conditional covariance matrix. It has a particularly strong impact on the long-run value of equity volatility while its effect on the variability of the government bonds 
is predominantly a short-run phenomenon. It is noteworthy, from the point of view of asset pricing models, that when modelled in a comprehensive multivariate framework, the inflation effects are strongest in the conditional second order moments and have little predictive power over the level of the excess return.

The inclusion of the inflation variable yields portfolio frontiers that present the investor with superior risk-return combinations, with the distribution of frontiers shifted to the left. The greatest risk reduction is enjoyed by investors who are interested in holding the minimum-variance portfolios. At higher levels of expected return, the frontiers begin to converge. Consequent ly, our optimal portfolios have very similar Sharpe Performance measures to those formed without taking account of the macro variable. We also show that using the time-varying conditional covariance matrix allows investors to reduce risk as opposed to working with a static unconditional matrix. Furthermore. assuming a static unconditional matrix will give a false representation of risk in many periods.

Finally, we solve for the opt imal vector of asset weights that the investor should hold in each period of the analysis. As usual, the unrestricted allocation is deemed to be excessively volatile so we concentrate on the port folio of assets that is constraimed to contain only non-negative quant it ies of each asset. Despite having simular SPI valnes as the portfollios formed without 
accounting for the inflation effect, the holdings of the assets do change. Equity continues to dominate the portfolio and on average accounts for $74 \%$ of the investment, strengthening its position by $4 \%$. The increase in the proportion of wealth allocated to equity may be partially explained by its large negative correlation with the inflation variable. The short-term bond enjoys increased importance, rising from $10 \%$ to $12 \%$ of the portfolio. In compensation, the long government bond attracts less investment and its mean holding falls from $20 \%$ to $14 \%$. These movements may be due to the fact the inflation influence on the second order moments was greatest for the long bond. 
Figure 7.1

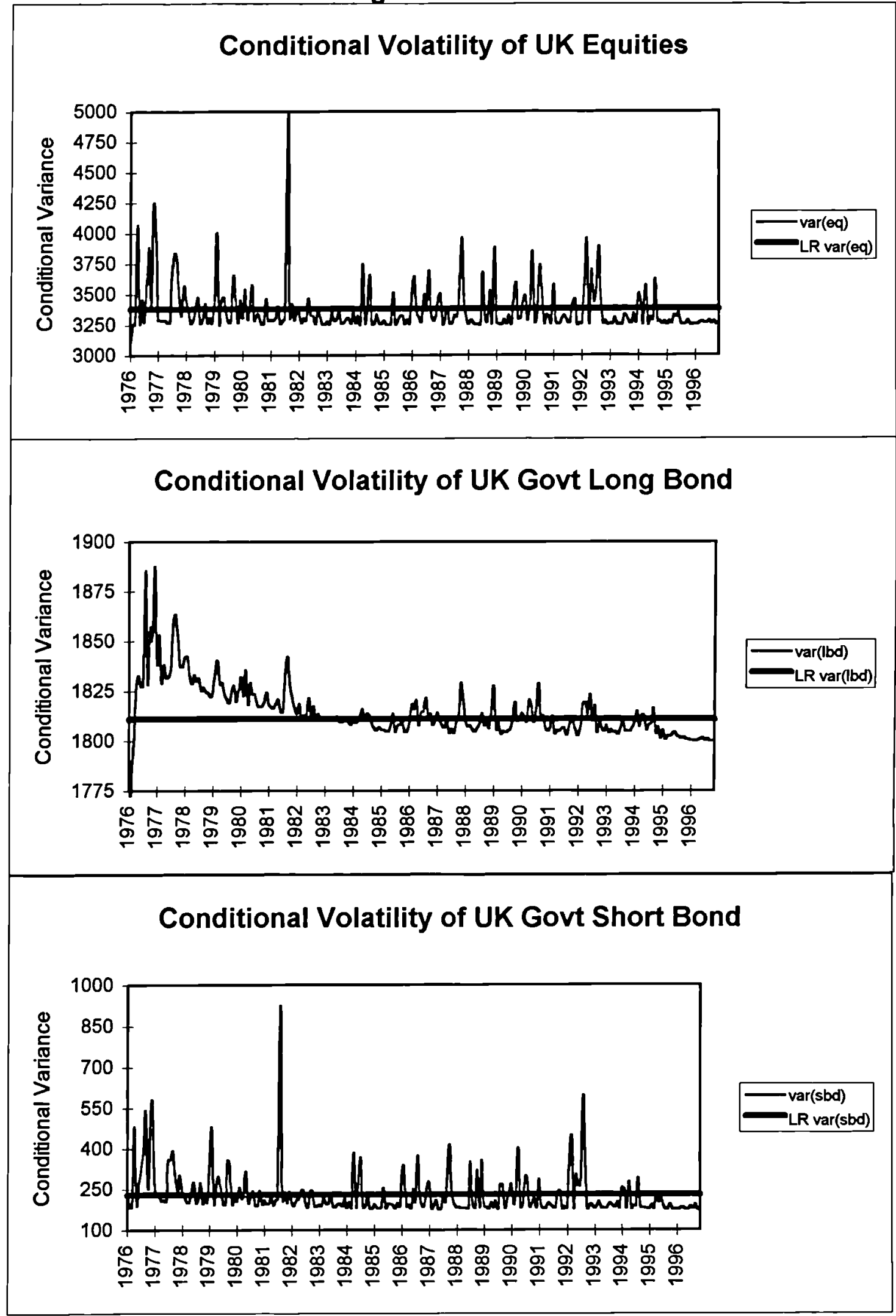




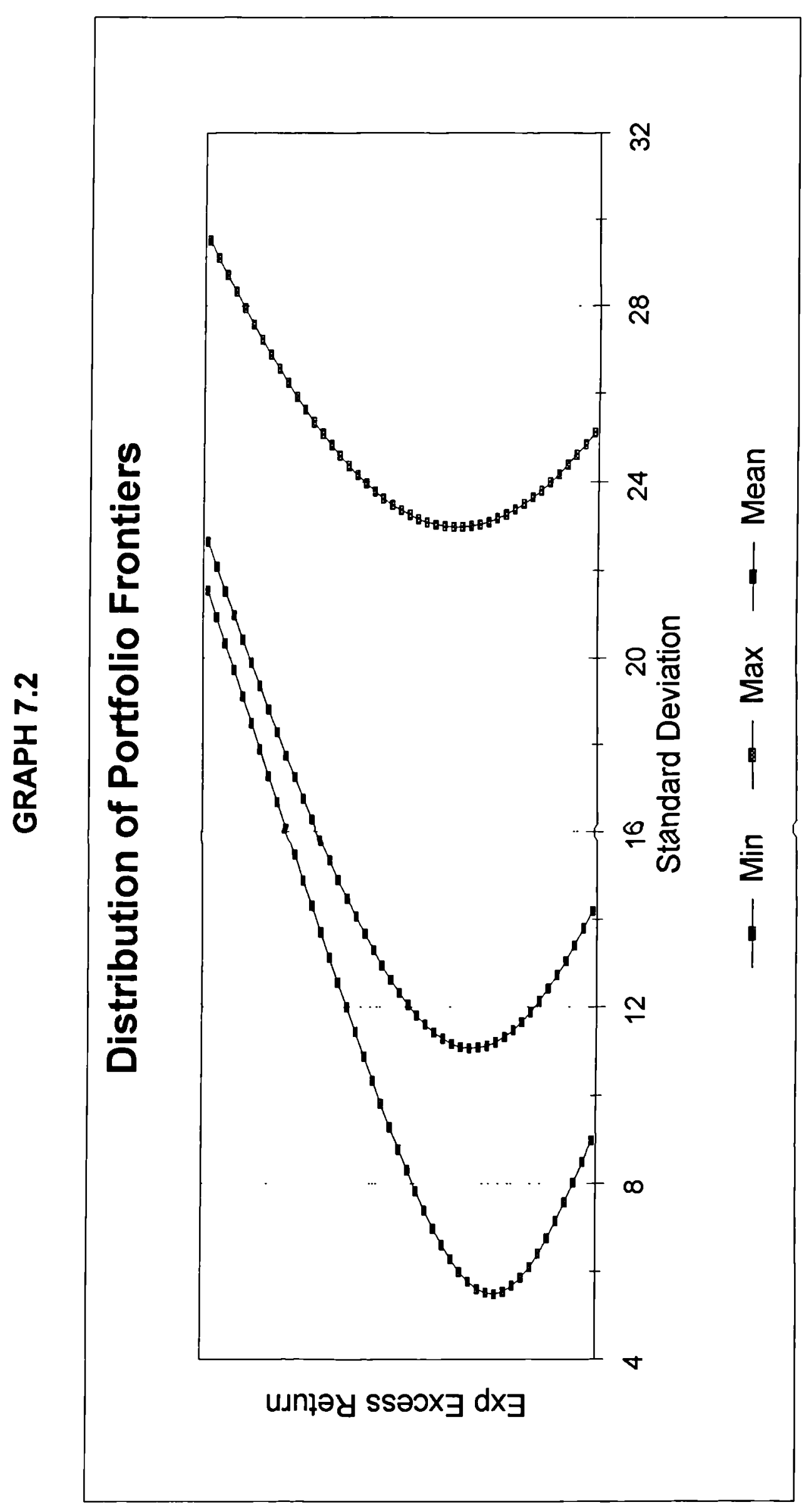

$\frac{\pi}{N}$ 


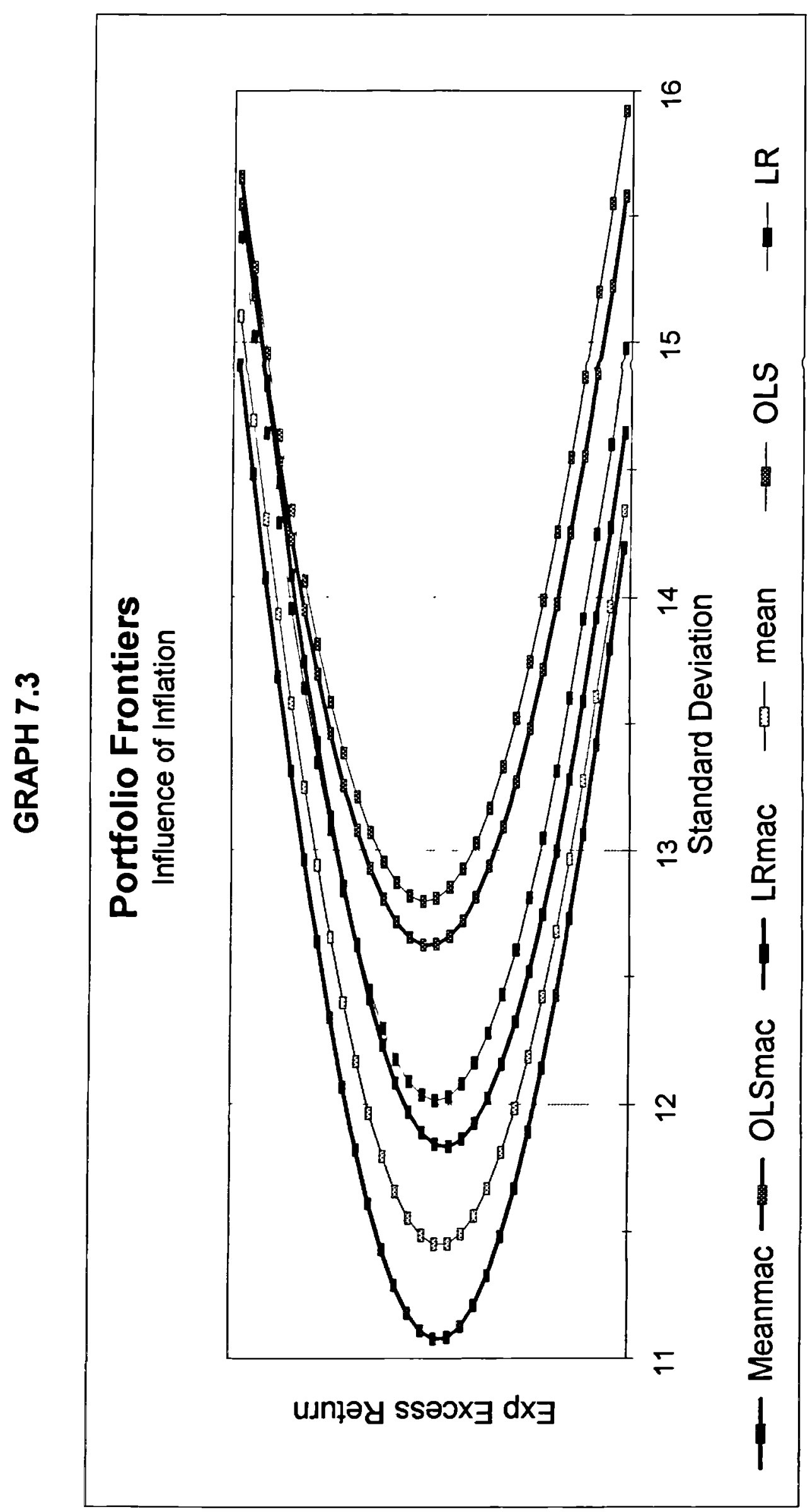




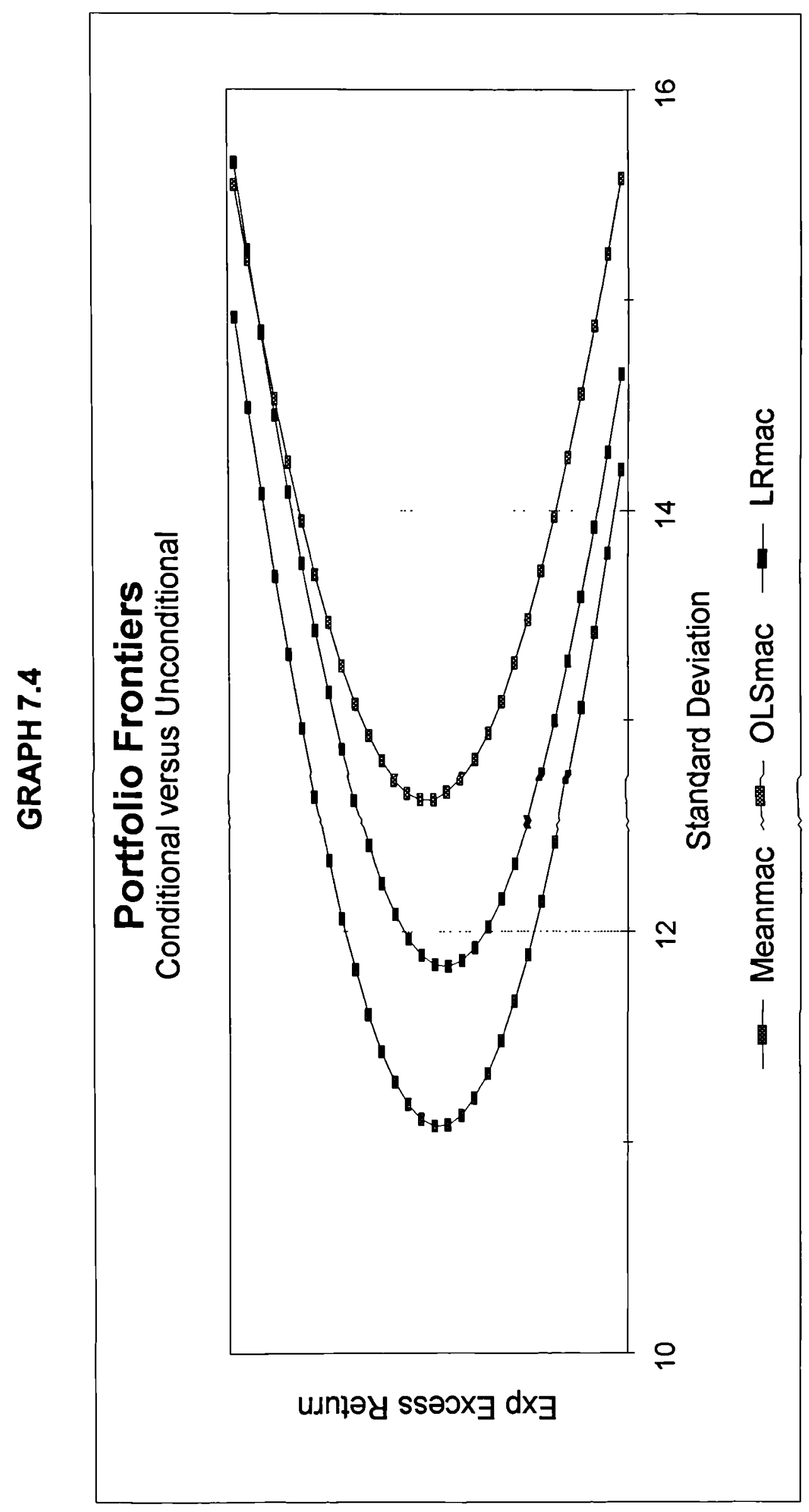

$\frac{0}{N}$ 
Figure 7.5

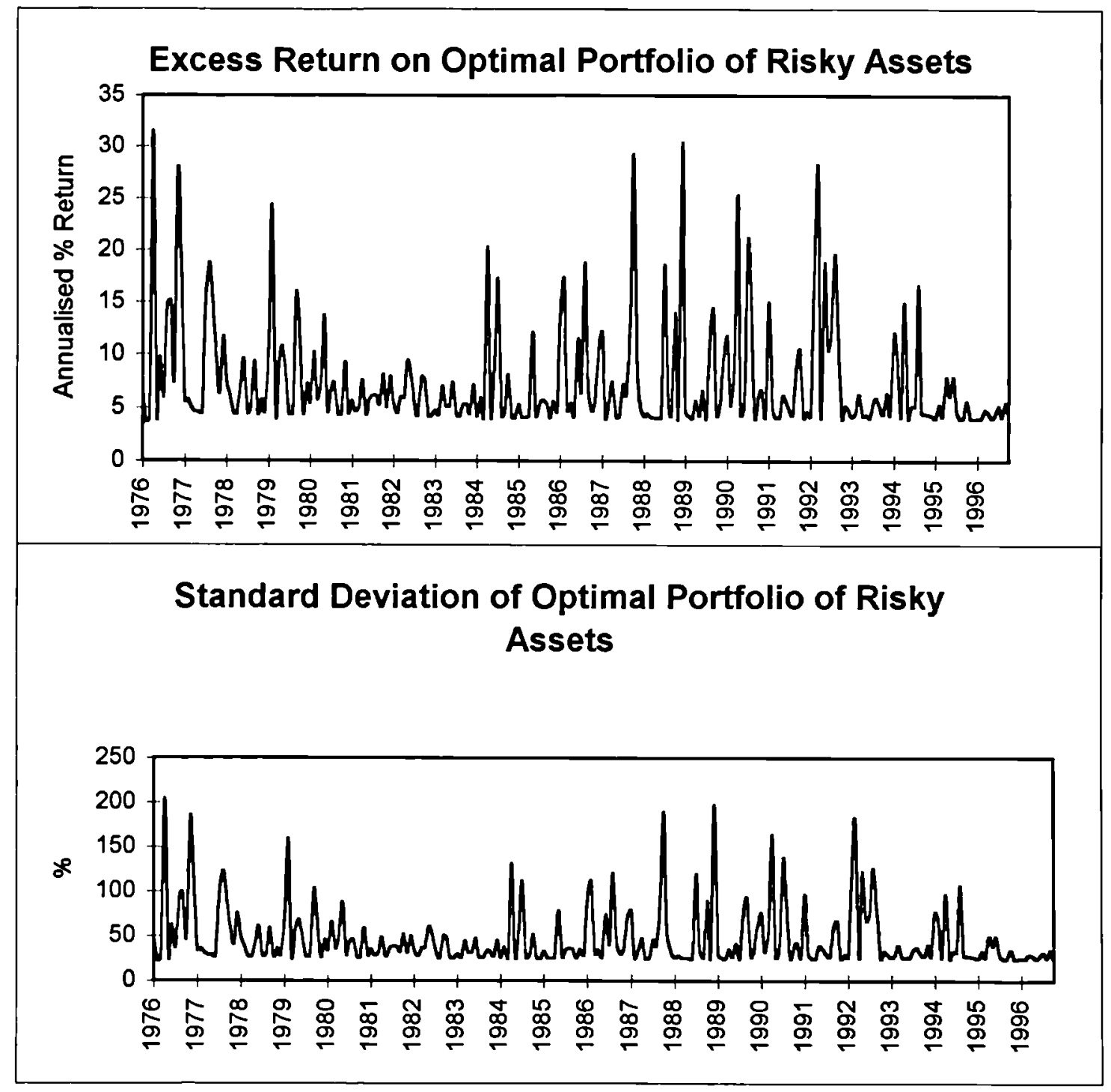




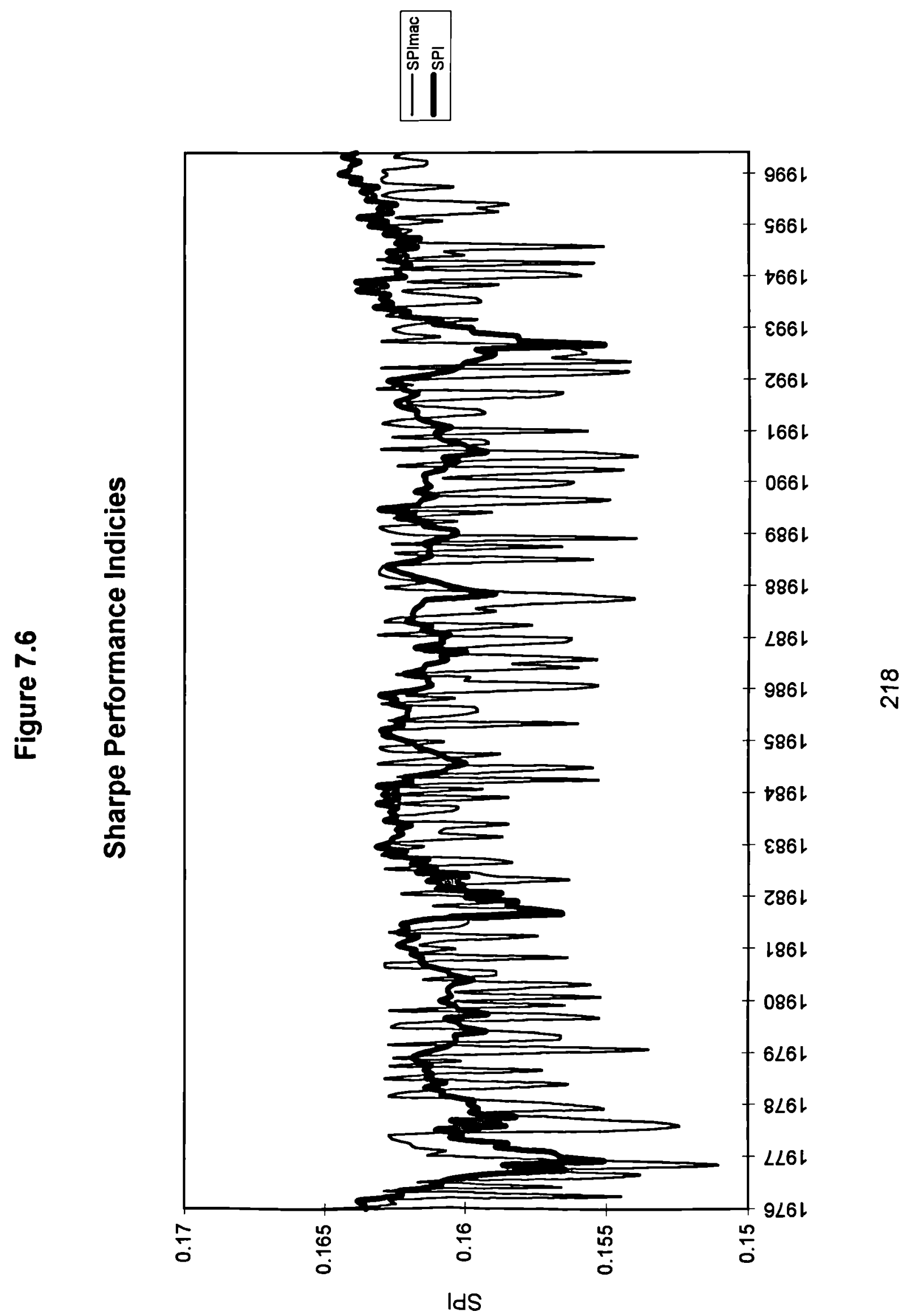



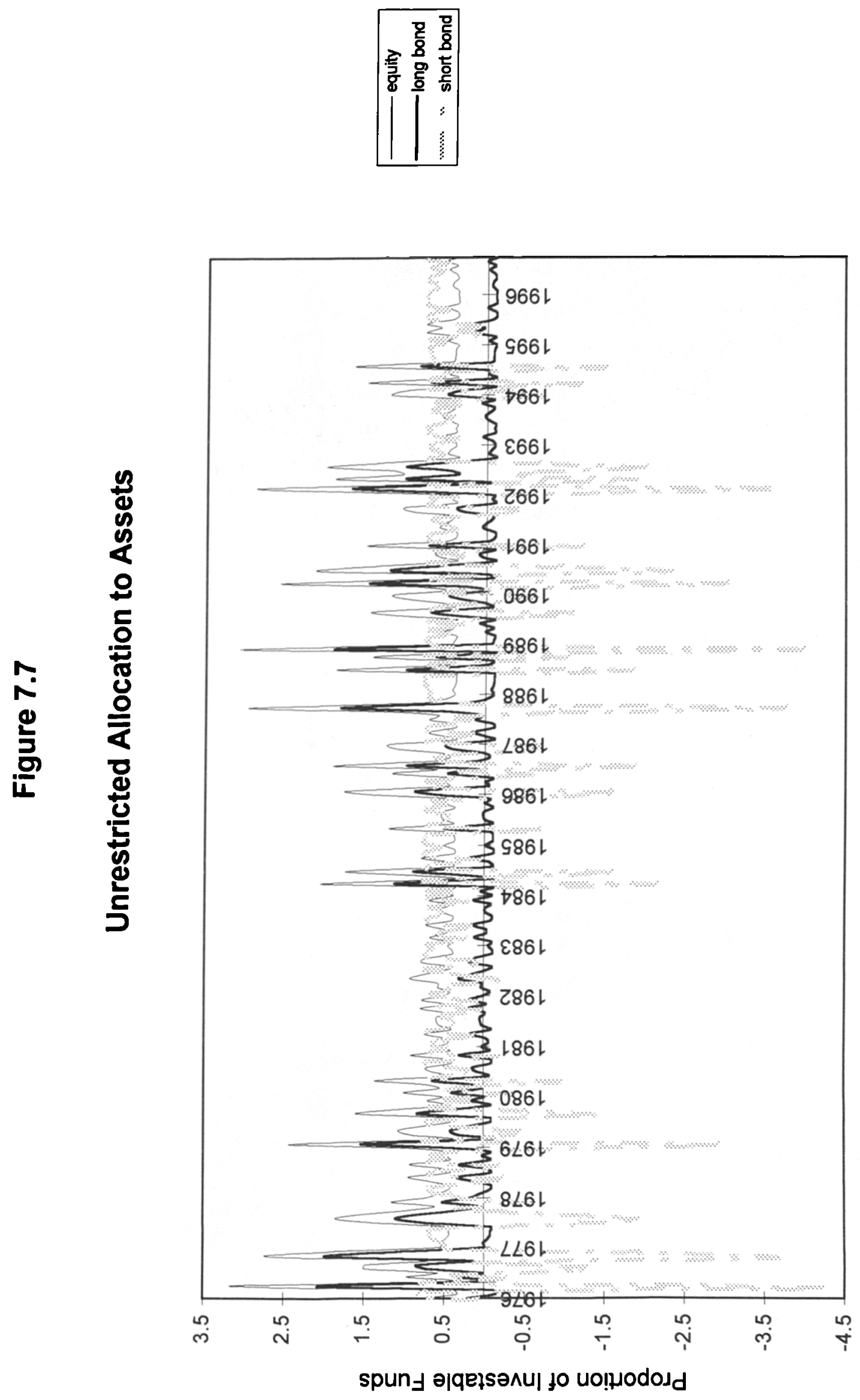

$\frac{\sigma}{\pi}$ 


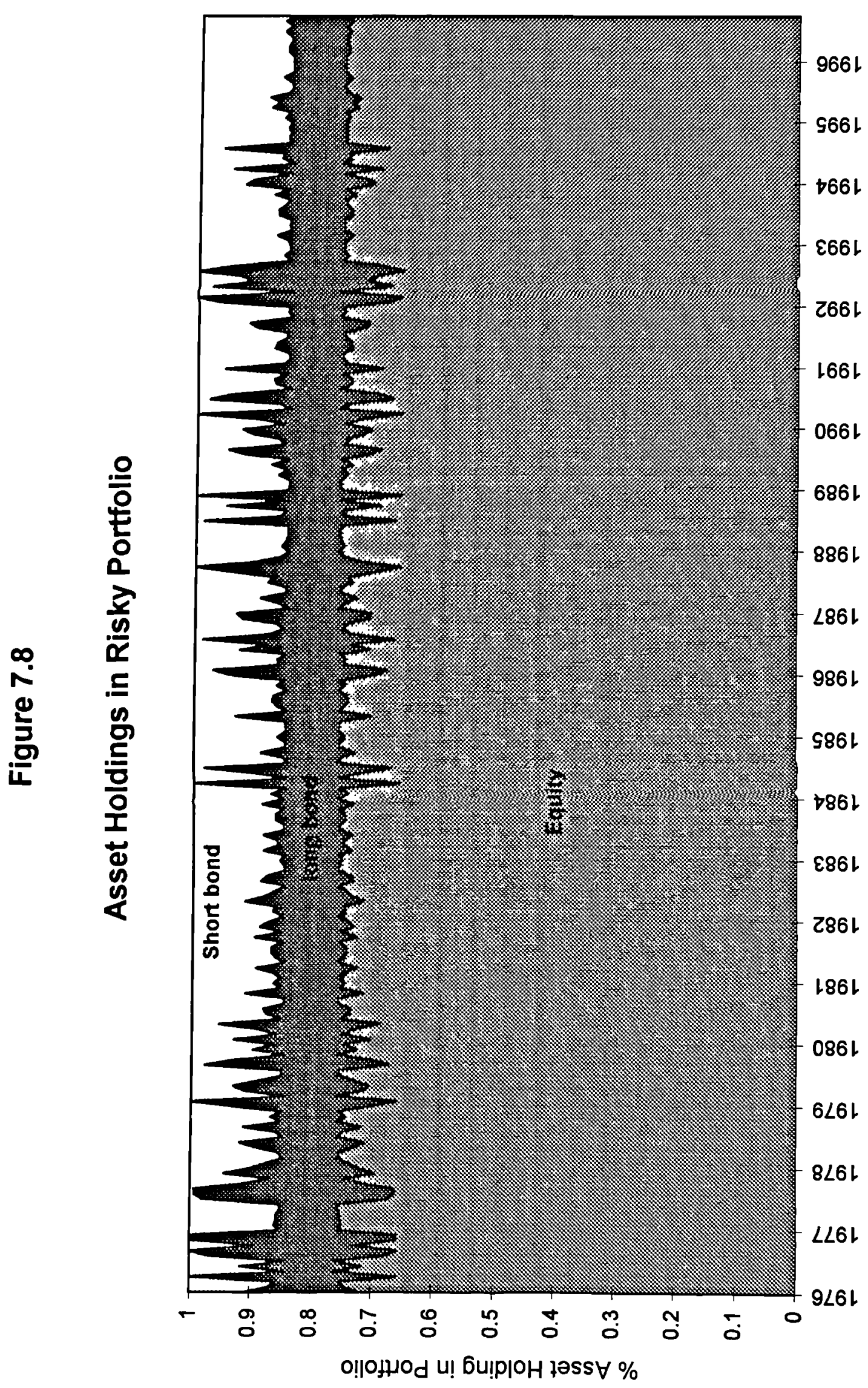




\section{Chapter 8}

\section{Macroeconomic Influences on}

\section{International Portfolio}

\section{Selection}

\subsection{Introduction}

In the previous chapter, we found that incorporating macroeconomic influences into our model enabled us to extract important information to predict future financial asset volatility. It was shown for an investor wishing to hold only domestic assets, that the change in the domestic inflation rate contained information that could be exploited to the investor's advantage in forecasting future asset return variances and covariances. Using these as 
inputs in the asset allocation decision helped the investor to reduce portfolio risk. In this analysis, we investigate if macroeconomic factors can exert an equally important influence on the portfolio selection process when the investor is allowed to diversify across international markets.

Our empirical work extends the analysis of chapter 5 . We take the perspective of a UK investor who has the opportunity to tactically allocate funds among three risky assets, namely domestic equity, domestic bonds and US equity. Now, we extend the analysis by introducing macroeconomic variables into the system and seek to extract information that will help us to predict both the level and volatility of future international asset price movements. Using the traditional CAPM model, we identify a set of macroeconomic factors that may potentially influence the asset allocation. In particular, the variables chosen are domestic and US inflation rates and the depreciation in the dollar-sterling exchange rate. Analysing the role of the inflation variables is of utmost concern since we have already seen its impact on the domestic portfolio and the desire to hedge against domestic inflation has been put forward by Sercu(1980), Adler and Dumas(1983) etc. as an explanation of the high degree of home bias in asset holdings.

Our findings show that when we allow international portfolio diversifcation, the inflation variables are no longer important in determining the asset allocation strategy, though there appears to be a long-run relationship 
between the conditional volatility of the foreign asset and the exchange rate. There is no evidence of short-run financial asset volatility being significantly influenced by the inclusion of these macro variables. Consequently, we conclude that when the opportunity to diversify across international assets exists, hedging against inflation is not an issue, contrary to the arguments of the aforementioned authors. In building a tactical asset allocation strategy, it is sufficient to model the excess returns of the financial assets, though it may be possible to improve portfolio performance by hedging against currency risk. Various versions of the model were estimated in case our results were being affected by the number of parameters in the formulation, but all models tell the same story. Holding a foreign asset provides an adequate hedge against inflation.

The remainder of the chapter proceeds as follows. Section 8.2 offers a theoretical justification for the choice of macroeconomic variables used in this analysis. The model is presented in section 8.3 together with a brief description of the data. Section 8.4 presents the results of the analysis while our concluding remarks are contained in section 8.5 .

\subsection{Macroeconomic Factors}

In this section, we seek to find a rationale for the choice of macroeconomic variables included in this application of the model. Focusing on the one- 
period static CAPM of Sharpe(1964) and Lintner(1965), lets suppose that we have a representative agent for the domestic country and for simplicity that his portfolio of risky financial assets contains one home asset and one foreign asset. This agent strives to maximise end-of-period real wealth, given information available at the beginning of the period.

$$
\operatorname{Max} U\left[E_{t}\left(W_{t+1}\right), V_{t}\left(W_{t+1}\right)\right], \quad U_{1}>0, U_{2}<0,
$$

where $E_{t}$ is the conditional expectation of end-of-period real wealth, $W_{t+1}$, and $V_{t}$ is the conditional variance. We can write

$$
E_{t}\left(W_{t+1}\right)=W_{t}+W_{t} \mathbf{x}_{t}^{\prime} E_{t} \mathbf{r}_{t+1}
$$

and its variance as:

$$
V_{t}\left(W_{t+1}\right)=W_{t}^{2} \mathbf{x}_{t}^{\prime} V_{t}\left(\mathbf{r}_{t+1}\right) \mathbf{x}_{t}
$$

where $\mathbf{x}_{t}$ is the $2 x 1$ vector of asset weights in the portfolio and $\mathbf{r}_{t+1}$ is a vector of asset real excess returns comprising the home asset return, $r_{t+1}^{d}$ and the real return on the foreign asset (expressed in the foreign currency) $r_{t+1}^{f}$. $V_{t}\left(\mathbf{r}_{t+1}\right)$ refers to the variance-covariance matrix of asset excess returns. The excess return on the portfolio of assets between $t$ and $t+1$ is given by: 


$$
R_{p, t+1}=\mathbf{x}_{t}^{\prime} \mathbf{r}_{t+1}
$$

Substituting (8.2) and (8.3) into (8.1) and maximising with respect to $\mathbf{x}_{t}$ gives the first order conditions:

$$
\frac{d U}{d x_{t}}=U_{1} W_{t} E_{t} \mathbf{r}_{t+1}+U_{2} W_{t}^{2} V_{t}\left(\mathbf{r}_{t+1}\right) \mathbf{x}_{t}=0
$$

where $U_{i}(i=1,2)$ denotes the partial derivative of $U$ with respect to the $i$ th argument.

Defining the coefficient of relative risk aversion, $\rho_{t}=-2 U_{2} W_{t} / U_{1}$ and re-arranging the above expression, we get the following condition:

$$
E_{t} \mathbf{r}_{t+1}=\rho_{t} V_{t}\left(\mathbf{r}_{t+1}\right) \mathbf{x}_{t}
$$

Now if we re-write these real returns in terms of observed nominal returns in the home currency and price level changes

$$
E_{t} \mathbf{r}_{t+1}=\left[\begin{array}{c}
r_{t+1}^{d} \\
r_{t+1}^{f}
\end{array}\right]^{\prime}=\left[\begin{array}{c}
i_{t+1}^{d} \\
i_{t+1}^{f}
\end{array}\right]^{\prime}\left[\begin{array}{cc}
P_{t}^{d} / P_{t+1}^{d} & 0 \\
0 & P_{t}^{f} / P_{t+1}^{f}
\end{array}\right]\left[\begin{array}{cc}
1 & 0 \\
0 & S_{t+1} / S_{t}
\end{array}\right]
$$

where superscript $d$ and $f$ refer to domestic and foreign variables respectively. $i_{t+1}$ denotes the nominal excess asset returns, $P_{t}$ represents the price 
index used to deflate nominal returns while $S_{t}$ is the foreign exchange price of one unit of the domestic currency.

Therefore, this theoretical background suggests that when analysing the impact of macroeconomic variables on international portfolio selection and working with nominal returns, we should consider the effects of both domestic and foreign inflation as well as the foreign exchange rate. Consequently, when we estimate our model empirically, we consider the potential effects of UK inflation, US inflation and the dollar-sterling exchange rate on the optimal mix of risky financial assets for the UK investor.

\subsection{The Model and Data}

\subsubsection{The Model}

We jointly model the financial asset returns and the macroeconomic factors using the M-GARCH( $(1,1)$ model of chapter 3 to estimate the time-varying covariance matrices of asset excess returns upon which the asset allocation decision depends. This allows us to assess the importance of macroeconomic effects on the variances and covariances of financial assets which enter as inputs into the portfolio selection process. As in the previous chapter, the vector of dependent variables in the conditional mean equation contains both returns on financial assets and a vector of macroeconomic variables. 
Consequently, both the conditional mean and second order processes of the model will be influenced by the macroeconomic factors contained in the analysis.

The model is a VAR(1) model in the conditional mean with the errors following a $\operatorname{GARCH}(1,1)$ process. Furthermore, we include a dummy variable for the October 1987 stockmarket crash in the equity equations of the model. The model is specified as follows:

$$
\begin{aligned}
\mathbf{z}_{t} & =\boldsymbol{\alpha}+\boldsymbol{\beta} \mathbf{z}_{t}{ }_{1}+\gamma d u m 87+\boldsymbol{\xi}_{t} \\
\boldsymbol{\xi}_{t} & \mid \Psi_{t}{ }_{1} \sim N\left(0, \mathbf{H}_{t}\right) \\
\mathbf{H}_{t} & =\mathbf{V}^{\prime} \mathbf{V}+\mathbf{A}^{\prime}\left(\mathbf{H}_{t}{ }_{1}-\mathbf{V}^{\prime} \mathbf{V}\right) \mathbf{A}+\mathbf{B}^{\prime}\left(\boldsymbol{\xi}_{t-1} \boldsymbol{\xi}_{t-1}^{\prime}-\mathbf{V}^{\prime} \mathbf{V}\right) \mathbf{B}
\end{aligned}
$$

where $\mathbf{z}_{t}=\left(\mathbf{r}_{t}, \mathbf{m}_{t}\right)^{\prime}, \mathbf{r}_{t}=\left(u k e q_{t}, u s e q_{t}, g b d_{t}\right)^{\prime}$ is a $3 x 1$ vector of excess returns on risky financial assets with $u k e q$, useq and $g b d$ representing UK equity, US equity and UK government bonds respectively while $\mathbf{m}_{t}=\left(\Delta \pi_{t}^{d}, \Delta \pi_{t}^{f}, \Delta S_{t}\right)^{\prime}$ is a $3 x 1$ vector of macroeconomic factors with the change in domestic and foreign inflation rates denoted by $\Delta \pi_{t}^{d}$ and $\Delta \pi_{t}^{f}$ and $\Delta S_{t}$ representing the depreciation in the dollar-sterling exchange rate ${ }^{1}$.

\footnotetext{
${ }^{1}$ After conducting Augmented Dickey-Fuller, Phillips-Peron and Stock-Watson tests for unit roots, we concluded that the inflation rates were $I(1)$ series. Therefore these variables enter the model in first difference format to ensure that all variables in the VAR structure are stationary.
} 


\subsubsection{Data}

This chapter uses financial asset return data for three risky financial assets. The risky assets in the investment opportunity set of the representative UK investor are UK equities, represented by the Financial Times All Share Index; US Equities represented by the S\&P Composite Index and UK government bonds represented by the FT British government stock index. The return on the foreign asset is converted into the domestic currency using the end of month dollar-sterling exchange rate. The data used in this chapter is annualised monthly total returns for each asset and furthermore each return is expressed in excess of the risk free rate of interest. The rate of return on UK government 30-day Treasury bills is taken as the riskless rate. The domestic inflation rate used is calculated from the UK Retail Price Index with the US equivalent being calculated from the US consumer price index. The data covers a sample period beginning in January, 1980 and finishing in September, 1996. All data was sourced from DATASTREAM.

\subsection{Estimation Results}

The model as specified in equation (8.8) was maximised subject to the Berndt, Hall, Hall and Hausmann (BHHH) algorithm. The results of the model are reported below with $t$-statistics in parentheses. 
8.4.1 Mean Matrices

$\alpha=\left[\begin{array}{c}8.85 \\ (1.62) \\ 7.95 \\ (1.14) \\ 1.86 \\ (0.58) \\ -0.02 \\ (0.4) \\ -0.02 \\ (0.5) \\ 0.001 \\ (0.24)\end{array}\right], \gamma=\left[\begin{array}{c}-393.66 \\ (0.47) \\ (1.46) \\ 0 \\ 0 \\ 0 \\ 0\end{array}\right]$




$\boldsymbol{\beta}=\left[\begin{array}{ccccccc}-0.10 & 0.11 & 0.12 & -1.03 & -5.74 & -59.93 \\ (0.7) & (0.64) & (0.38) & (0.08) & (0.31) & (0.14) \\ 0.03 & -0.02 & -0.09 & -6.73 & -24.16 & 59.84 \\ (0.18) & (0.11) & (0.25) & (0.38) & (1.04) & (0.10) \\ -0.02 & -0.03 & 0.07 & -2.26 & -13.19 & -80.03 \\ (0.19) & (0.32) & (0.57) & (0.34) & (1.50) & (0.37) \\ 0.001 & -0.002 & -0.001 & 0.30 & 0.30 & 5.41 \\ (0.63) & (1.33) & (0.41) & (1.57) & (1.56) & (1.60) \\ 0.002 & 0.000 & 0.000 & 0.07 & 0.42 & -0.60 \\ (0.28) & (0.08) & (0.39) & (0.73) & (3.62) & (0.25) \\ 0.000 & 0.000 & 0.000 & -0.003 & -0.005 & 0.08 \\ (0.59) & (0.61) & (0.45) & (0.48) & (0.53) & (0.41)\end{array}\right]$

\subsubsection{Covariance Matrices}

Since the V, A and $\mathbf{B}$ matrices are symmetric, only the lower triangle is reported here for clarity of exposition. 


$V=\left[\begin{array}{ccccccc}52.02 & & & & \\ (20.0) & & & & & \\ 29.87 & 52.33 & & & & & \\ (6.4) & (15.23) & & & & & \\ 13.82 & -2.08 & 20.60 & & & \\ (9.98) & (1.08) & (16.31) & & & \\ -0.06 & -0.02 & -0.06 & 0.42 & & \\ (0.86) & (0.35) & (1.02) & (9.21) & & \\ 0.01 & -0.04 & 0.01 & 0.04 & 0.27 & \\ (0.4) & (0.92) & (0.16) & (0.69) & (4.18) & \\ 0.002 & 0.02 & -0.001 & -0.002 & -0.001 & 0.01 \\ (0.97) & (7.85) & (0.42) & (0.60) & (0.05) & (8.87)\end{array}\right]$




$$
\mathbf{A}=\left[\begin{array}{ccccccc}
0.35 & & & & \\
(0.15) & & & & & & \\
-0.15 & 0.25 & & & & & \\
(0.08) & (0.10) & & & & & \\
-0.49 & 0.27 & 0.33 & & & & \\
(0.36) & (0.31) & (0.16) & & & \\
-0.13 & 0.07 & -0.06 & 0.27 & & \\
(0.19) & (0.17) & (0.08) & (0.14) & & \\
-0.31 & 0.15 & -0.25 & -0.08 & 0.33 & \\
(0.26) & (0.15) & (0.24) & (0.10) & (0.16) & \\
0.002 & -0.001 & 0.001 & -0.005 & 0.03 & 0.06 \\
(0.07) & (0.07) & (0.02) & (0.11) & (0.83) & (0.03)
\end{array}\right]
$$




$$
\mathbf{B}=\left[\begin{array}{ccccccc}
0.03 & & & & \\
(0.19) & & & & & \\
0.05 & 0.08 & & & & \\
(0.30) & (0.42) & & & & & \\
-0.01 & -0.003 & 0.01 & & & \\
(0.28) & (0.03) & (0.23) & & & \\
0.002 & -0.001 & 0.001 & 0.23 & & \\
(0.78) & (0.60) & (0.13) & (0.76) & & \\
0.001 & -0.001 & -0.002 & -0.03 & 0.12 & \\
(0.49) & (0.48) & (0.48) & (0.15) & (0.43) & \\
0.0001 & -0.0002 & 0.0001 & 0.001 & -0.0004 & 0.10 \\
(0.41) & (1.55) & (0.59) & (0.10) & (0.04) & (0.40)
\end{array}\right]
$$

\subsubsection{Discussion of Results}

As usual, we find that the mean parameters are predominantly insignificant, providing further evidence of the extreme difficulty in predicting future financial asset price movements. Concentrating on the conditional second order moments, we find that the own variance elements of the long-run matrix, V, are significantly different from zero as are the covariances between UK and US equity $\left(V_{21}\right)$ and the two domestic assets $\left(V_{31}\right)$. This is consis- 
tent with our other study in chapter 5 which also found contagion effects between these markets. Once more, the UK bond market appears to be segmented from the US market and is unaffected by its volatility spillovers. The macroeconomic factors fail to exert an influence on the conditional variances and covariances of the financial assets. However the inclusion of the respective inflation rates has no influence on the determination of the long-run volatilities. Therefore, it appears that holding the foreign asset is an adequate hedge against inflation risk. There is evidence of a long-run effect from the dollar-sterling exchange rate on the US equity, which is captured by $V_{62}$. This suggests that the performance of the portfolio may be improved by hedging against exchange rate movements as presently foreign exchange risk is a significant determinant of the long-run volatility of the foreign asset.

The short-run matrices find no support for the inclusion of macroeconomic variables in this analysis. In fact, none of the parameters in the $\mathbf{A}$ or B are statistically different from zero. While this may be seen as evidence against our model, we know that based on earlier results that this may be further interpreted as evidence of the foreign asset providing an adequate hedge against inflation movements.

One potential worry about these results may be that the relatively large parameter standard deviations could be due to the convergence procedure 
of the M-GARCH model being unable to cope with such a vast number of parameters. To allay our fears, we re-estimated the model with $\mathbf{z}_{t}$ reduced from a $6 x 1$ to a $5 x 1$ vector. This was achieved by replacing the individual inflation rates by the ratio of UK/US inflation rates. However, the results were unchanged. Exactly the same elements were statistically different from zero, confirming the contagion effects noted earlier and the fact the foreign asset provides a hedge against inflation.

Finally, we reduced the number of macroeconomic factors to just a single variable, namely domestic inflation. Again the results reported above were confirmed, though there was some marginal significance in a number of the short-run parameters linking the financial assets. However, the conclusion that the foreign asset provides a hedge against inflation risk is strengthened as none of the parameters in the final rows of $\mathbf{V}, \mathbf{A}$ or $\mathbf{B}$ are found to be statistically significant. These models, therefore, fail to find any evidence to support the contention of Sercu(1980), Adler and Dumas(1983) and others that the high concentration of domestic assets in investors' portfolios is motivated by a desire to hedge domestic inflation but is consistent with the finding of Uppal(1993) who argues that this explanation of the home bias puzzle is only valid for implausibly low values of the coefficient of relative risk aversion. Our results show that holding a foreign asset in the portfolio provides an adequate hedge against inflation. 


\subsection{Conclusion}

The aim of this chapter was to analyse the effects of macroeconomic variables on the tactical asset allocation strategy of a UK investor who is interested in diversifying across international markets. Starting from a standard mean-variance framework, we identified a set of macroeconomic factors that may potentially influence the portfolio selection decision of an investor. These factors were domestic inflation, foreign inflation and the foreign exchange rate. We assessed their impact on the asset returns by jointly modelling these variables with a set of financial asset excess returns comprising of UK equity, US equity and UK government bonds.

In contrast to our study of the domestic portfolio, there is no extra information to be derived from the inclusion of the inflation variables. None of the macro factors exert a statistically significant impact on the conditional mean and indeed only the dollar-sterling exchange rate exerts any influence on the conditional second order moments of the process. It appears that holding a foreign asset provides an adequate hedge against inflation risk. This observation coupled with the fact that the short-run dynamics of the process are no longer significantly different from zero leads us to the conclusion that when selecting the optimal portfolio of international risky financial assets, we should not be concerned about inflation effects. It is sufficient to model the asset excess returns and use these conditional variances and 
covariances as inputs in the portfolio selection procedure, as in chapter 5 . However the portfolio performance may be improved by hedging against currency risk, as volatility in the foreign exchange market contributes to the volatility of the foreign asset.

Other more parsimonious versions of the model were estimated to ensure that the insignificant parameters were not a result of the M-GARCH model being incapable of dealing with the number of parameters. We repeated the analysis by first combining the inflation rates into a single variable to leave five dependent variables and secondly, removing all macro factors except the domestic inflation variable. However, the results were unambiguous. Exactly the same parameters were significantly different from zero as reported above. There is no evidence that inflation plays any role in the determination of the conditional asset variances and covariances. Therefore, our results find no evidence to support the claims of Sercu(1980), Adler and Dumas(1983) etc. that the observed degree of home country bias is a result of a desire to hedge domestic inflation. 


\section{Chapter 9}

\section{Conclusion}

\subsection{Review of Results}

The aim of the thesis was to utilise recently developed econometric techniques to address important issues in the world of financial economics. Our central aim was to build an asset allocation model that incorporated real istic features of financial markets. Our framework develops the portfolio management theory of Markowitz (1952. In his mean-variance analysis, Markowitz assumed that investors are concerned with minimising the riskiness of the total return on the portfolio subject to achieving a target rate of return.

As we have seen, in practice financial asset excess returns are not forecastable (they are virtually serially independent, therefore the emphasis 
should be on minimising the variance of the portfolio's return by choosing appropriately the proportions in which each asset is held in the portfolio. Early work on asset allocation worked within the static framework introduced by Markowitz(1952). In particular, the covariance matrix of returns was assumed to be constant. There is now ample evidence that this assumption is incorrect, and that the covariance matrix of returns is time varying. The first attempts to take this into account assumed that the covariance matrix was sufficiently slowly changing that it could be estimated by the unconditional matrix of past returns and then treated as though it would be constant for a fixed period in the future. In the absence of transaction costs, this implies that the optimal portfolio should be re-balanced each period. Unlike returns, it is possible to forecast changes in the covariance matrix over time. This suggests that the aim of tactical asset allocation should be to exploit the regularities in the covariance structure of returns with the aim of reducing risk.

The development of the family of ARCH (Engle(1982)) and GARCH (Bollerslev(1986)) models has made it possible to allow the covariance matrix to be continuously changing. They also help to capture other features of asset returns such as thick tails and volatility clustering. Multivariate (G)ARCH models are ideally suited to the task of building asset allocation models since the covariance terms are vital to the successful implementa- 
tion of any portfolio selection strategy. However, in the past these models have often been ignored due to the large number of parameters to be estimated simultaneously. Recent advancements in computing power now make it possible to fully exploit them.

The first original contribution in this thesis is a new variant of a parsimonious parameterisation of the multivariate GARCH model. The main innovation is that we write the conditional second order moments in errorcorrection format and this enables us to easily disentangle long- and shortrun effects. This distinction becomes very important in building tactical asset allocation models. By formulating the conditional covariance matrices in this way, we can decide more easily if the short-run dynamics make a useful additional contribution, and if the increased generality a parameter offers is worth the additional computational burden. As the number of assets increases it may be necessary to further restrict these matrices by, for example, setting some of the coefficients to zero and closing off some of the transmission channels in the long and short run.

The remaining contributions of this thesis lie in the application of this model to issues in financial economics. We focus on portfolio selection problems. Chapter 4 begins the investigation of building tactical asset allocation strategies based on risk minimisation. Here, we look at the problem from the perspective of a UK investor wishing to hold only domestic assets. Our 
initial results are encouraging. There is evidence of significant short-run deviations from the long-run value of volatility for each of the three assets under review. The portfolio frontiers (and hence the location of the optimal portfolio of risky assets) exhibit a great deal of variation over time in both shape and location and it is shown that a considerable reduction in portfolio risk can be achieved by using the conditional covariance matrix instead of a constant, unconditional, estimate. We find that in identifying the asset proportions necessary for the investor to hold the optimal portfolio often required the short sale of at least one asset. Since many investors are unable or unwilling to adopt such positions or may be restricted by law from doing so, e.g. UK mutual fund managers, it becomes necessary to allocate investment funds such that only non-negative asset holdings are allowed. The mean asset holdings in the optimal portfolio are $70 \%$ to equity, $20 \%$ to long government bonds and $10 \%$ to short government bonds. We also crucially find that the time-varying portfolios systematically offer a substantial risk reduction while achieving returns on a par with a more conventional buy and hold portfolio.

Chapter 5 looks at the tactical asset allocation problem from the perspective of both UK and US investors. Both can invest in domestic assets and the equity of the other country. Firstly, we look at the problem of home country bias. Our results confirm that investors can reap additional 
benefits from international diversification. Survey results tell us that UK and US investors hold about $18 \%$ and $6 \%$ of their wealth respectively in non-domestic assets. Our results suggest these numbers should be $25 \%$ and $20 \%$, even when there is only one foreign asset. The home-bias puzzle seems therefore to be more acute for US than UK investors and there seems to be more potential gains from increased international diversification for the US investor. We show that the location of the investor is important in determining the investment performance of the portfolio. The US investor enjoys a 'risk-return' advantage which is demonstrated by the distribution of portfolio frontiers lying to the left of the UK distribution and is confirmed by the higher average Sharpe Performance Index. There is also evidence of contagion effects across markets. In the long run, there are volatility spillovers between all markets. In the short run we find that volatility in the UK equity market is influenced by the US stockmarket but volatility is not transmitted in the opposite direction. Also, stock market volatility spills over to the domestic bond market but bond markets are segmented from foreign equity markets with short-run deviations being determined by domestic events.

Chapter 6 extends the analysis of the previous chapter for the UK investor to increase the available investment opportunity set. Parameterisation problems are overcome by adopting a two-stage approach. Firstly we 
form optimal portfolios of global bonds and european equity and in the second stage, these enter as a single asset. Our results show that the optimal portfolio is dominated by the home equity and that the UK bond is also an important asset - in fact this is the only asset which is never omitted from the portfolio. However, on average, these two assets constitute only $57 \%$ of the portfolio, thereby showing that foreign assets should account for the remaining $43 \%$. In the mean optimal portfolio six foreign assets are held in positive quantities along with the two domestic assets. US, French and German equity make up $40 \%$ of the portfolio. This result shows that the home bias problem is more acute than thought in the previous chapter and increased international diversification would benefit the UK investor. Secondly, introducing more assets moves the frontier significantly towards the origin and offers the investor the opportunity to enjoy higher levels of return for each risk level and hence generates optimal portfolios with much larger SPI values than in our previous studies. Furthermore we find evidence of volatility contagion effects between individual bond markets and stock markets. However, the equity markets of Japan and Europe are segmented from each other and bond markets appear to be segmented from stock markets. Since volatility sources for bonds and stocks appear to be different, diversification benefits are increased by holding both in a portfolio of risky assets. 
In chapter 7 , we extend the analysis of chapter 4 to allow macroeconomic phenomena to influence the asset allocation decision through their conditional covariances with the financial assets under review. This is a response to the growing literature advocating the use of macroeconomic factors and financial ratios to predict future asset price movements. Our model provides a neat way to incorporate these effects as we can jointly model the financial asset returns and the macroeconomic variables. This allows the variable to exert an influence on both the first and second order moments of the process and hence on the asset allocation through its covariance with each of the financial assets. From an econometric modelling point of view, we discover that each of the second order moment matrices should be specified as a full symmetric matrix in order to minimise the time lag with which the macro variable exerts its influence. The inclusion of the inflation variable yields portfolio frontiers that present the investor with superior risk-return combinations, especially for the investor who is interested in identifying the minimum-variance portfolio. Concentrating on the constrained asset allocation strategy, we find that equity dominates the portfolio in every period, with an average holding of $74 \%$. The remainder of the portfolio consists of the two government bonds, which have mean holdings of $14 \%$ and $12 \%$ respectively. Our results show that when a UK investor builds an entirely domestic portfolio of risky assets, then inflation 
needs to be taken into account.

Chapter 8 looks at the effects of macroeconomic variables, and in particular inflation, on the allocation decision when the investor has an internationally diversified portfolio. In this respect, we extend the analysis of chapter 5 for the UK investor to account for both domestic and foreign inflation as well as changes in the sterling-dollar exchange rate. In contrast to the domestic portfolio analysis of the previous chapter, we find that there is no extra information to be derived from the inclusion of the inflation variables. None of the macro factors exert a statistically significant impact on the conditional mean and indeed only the dollar-sterling exchange rate exerts any influence on the conditional second order moments of the process. It appears that holding a foreign asset provides an adequate hedge against inflation risk. This observation, coupled with the fact that the short-run dynamics of the process are no longer significantly different from zero, leads us to the conclusion that when selecting the optimal time-varying portfolio of international risky financial assets, we should not be concerned about inflation effects. It is sufficient to model the asset excess returns, as in chapter 5. We should qualify this by admitting that the portfolio performance may be improved by hedging against currency risk. Therefore, our results find no evidence to support the claims of Sercu(1980), Adler and Dumas(1983) etc. that the observed degree of home country bias is motivated by the 
investor's desire to hedge domestic inflation.

\subsection{Future Research}

In this thesis, we have shown that our econometric model has many applications in the world of financial economics. Many issues have been inadequately dealt with in the past due to the lack of multivariate models capable of incorporating time-varying measures of risk. Therefore, in order to fully exploit the potential of our model from chapter 3 , there are many avenues of research available.

Immediately obvious is the investigation of the importance of hedging against risk in the foreign exchange market. Summarising our findings on optimal asset allocation, we would advocate that a UK investor should hold an internationally diversified portfolio of assets, with as large as possible an investment opportunity set considered. This has many advantages. We saw the superior performance of the internationally diversified portfolios and also chapter 8 shows that holding non-domestic assets in the portfolio provides a hedge against domestic inflation. However, one outstanding issue is whether or not to hedge against currency fluctuations. Again chapter 8 tells us that it may lead to an improvement in portfolio performance. Such a project is itself worthy of a $\mathrm{PhD}$ thesis as there are many questions to be answered. Should we hedge or not? Eun and Resnick(1994) suggest that investor 
location is an important issue in whether currency hedging yields greater portfolio performance. An emerging consensus in the literature is that we should hedge, see Glen and Jorion(1993), Bakaert and Hodrick(1992) etc. but then what is the optimal hedge ratio? Should we adopt a complete hedge or should we continue to bear some exchange rate risk? This is where the concensus breaks down, see Black(1989), Eaker and Grant(1990) and Gardner and Wuillord(1995). Therefore a thorough investigation of the benefits of hedging, whether or not investor location is an important factor and the degree to which investors should hedge, appears to be a worthwhile project to undertake.

An interesting potential advantage of EMU is that investors from countries within this zone will in future be able to diversify internationally without bearing any foreign exchange risk. Given the importance of French equity in the optimal portfolio of the UK investor (see chapter 6), this could be an important argument for joining the single currency.

Secondly, it should be interesting to perform a more rigourous analysis of contagion effects between world stockmarkets. This could become a live issue very soon with the Asian and Russian crisis threatening to throw some of the major world markets into turmoil. Questions such as "Which markets lead others?" and "Is the degree of volatility spillover from market to market constant or time-varying?" are potentially important for investors wishing 
to diversify internationally.

Thirdly, it would be interesting to know if diversifying across different industries can offer comparable levels of risk reduction as international diversification. Such diversification could be achieved without foreign exchange risk. Even if the reduction is not as great, it may appeal to investors who have low currency risk tolerance.

Finally, outside of asset allocation issues, there are other areas of finance that may be explored using the M-GARCH model developed here. Current work in progress demonstrates its flexibility by using it to investigate if the level of government debt in EU countries needs to be regulated. Using an arbitrage theory of interest rate determination, we are able to analyse the influence of domestic fiscal and monetary policy on the term structure of interest rates. This is an important policy issue for the EU with the single currency about to become a reality.

Another application of this model allows us to consider the potential influences of the risk premium between EU countries and Germany using both long-term and short-term interest rates. Again, we propose to use measures of fiscal and monetary policy to shed some light on the sources of the risk premium. 


\section{Appendix 1}

Equation of the Portfolio frontier in expected excess return, standard deviation space is

$$
s d_{t}=\sqrt{\frac{a_{t}-2 b_{t} R_{p t}+c_{t} R_{p t}^{2}}{a_{t} c_{t}-b_{t}^{2}}}
$$

Equation of the Capital Market Line

$$
s d_{t}=m_{t} R_{p t}
$$

At the point of tangency, the slopes of these two functions must be equal.

This implies that

$$
\begin{aligned}
m_{t} & =\frac{1}{2}\left(\frac{a_{t}-2 b_{t} R_{p t}+c_{t} R_{p t}^{2}}{a_{t} c_{t}-b_{t}^{2}}\right)^{\frac{-1}{2}}\left(\frac{2 c_{t} R_{p t}-2 b_{t}}{a_{t} c_{t}-b_{t}^{2}}\right) \\
& =\frac{c_{t} R_{p t}-b_{t}}{\left(a_{t}-2 b_{t} R_{p t}+c_{t} R_{p t}^{2}\right)^{\frac{1}{2}}\left(a_{t} c_{t}-b_{t}^{2}\right)^{\frac{1}{2}}}
\end{aligned}
$$


Replace $m_{t}$ in equation (A.2) and solve the simultaneous equation system

$$
\begin{array}{ll}
\left(\frac{a_{t}-2 b_{t} R_{p t}+c_{t} R_{p t}^{2}}{a_{t} c_{t}-b_{t}^{2}}\right)^{\frac{1}{2}} & =\left(\frac{c_{t} R_{p t}-b_{t}}{\left(a_{t}-2 b_{t} R_{p t}+c_{t} R_{p t}^{2}\right)^{\frac{1}{2}}\left(a_{t} c_{t}-b_{t}^{2}\right)^{\frac{1}{2}}}\right) R_{p t} \\
a_{t}-2 b_{t} R_{p t}+c_{t} R_{p t}^{2} & =c_{t} R_{p t}^{2}-b_{t} R_{p t} \\
b_{t} R_{p t} & =a_{t} \\
R_{p t} & =\frac{a_{t}}{b_{t}}
\end{array}
$$




\section{Bibliography}

[1] Adler, M. and B. Dumas(1983). International Portfolio Choice and Corporation Finance: A Synthesis. Journal of Finance, 38, 3. Pp 925984 .

[2] Agmon, T. and D. Lessard(1977). Investor Recognition of Corporate International Diversification. Journal of Finance, 32, 4. Pp 1049-1055.

[3] Asprem, M.(1989). Stock Prices, Asset Portfolios and Macroeconomic Variables in Ten European Countries. Journal of Banking and Finance, 13. $\operatorname{Pp}$ 589-612.

[4] Bailey, W. and R. Stulz(1990). Benefits of International Diversification: The Case of Pacific Basin Stock Markets. Journal of Portfolio Management, Summer. Pp 57-61.

[5] Baxter, M. and U.J. Jermann(1997). The International Diversification Puzzle is worse than you think. American Economic Review, 87, 1. Pp 170-180. 
[6] Baillie, R.T. and T. Bollerslev(1990). A Multivariate Generalized ARCH approach to modeling risk premia in Forward Foreign Rate Markets. Journal of International Money and Finance, 9. Pp 309-324.

[7] Bekaert, G. and R. Hodrick(1992). Characterizing Predictable Components in Excess Returns on Equity and Foreign Exchange Markets. Journal of Finance, 47. Pp 467 - 509.

[8] Bera, A.K. and M.L. Higgins(1993). ARCH Models: Properties, Estimation and Testing. Journal of Economic Surveys, 7. Pp $305-366$.

[9] Bera, A.K. and J.S. Roh(1991). A Moment Test of the Constancy of the Correlation in the Bivariate GARCH Model. Mimeo, Department of Economics, University of Illinois.

[10] Berndt, E.R., B.H. Hall, R.E. Hall and J.A. Haussman(1974) Estimation and Inference in Nonlinear Structural Models. Annals of Economic and Social Management, 4. Pp 653 - 665.

[11] Best, M.J. and R.R. Grauer(1991). On the Sensitivity of MeanVariance Efficient Portfolios to changes in Asset Means: Some Analytical and Computational Results. Review of Financial Studies, 4. Pp $315-342$. 
[12] Black, F.(1989). Universal Hedging: Optimizing Currency Risk and Reward in International Equity Portfolios. Financial Analysts Journal, 45. Pp $16-22$.

[13] Bodie, Z.(1976). Common Stocks as a hedge against Inflation. Journal of Finance, 31. Pp 459-470.

[14] Bollerslev, T.(1986). A Generalized Autoregressive Conditional Heteroskedasticity. Journal of Econometrics, 31. Pp 307 - 327.

[15] Bollerslev, T.(1990). Modelling the coherence in short-run nominal exchange rates: A Multivariate Generalized ARCH approach. Review of Economics and Statistics, 72. Pp 498-505.

[16] Bollerslev, T., R. Chou and K.F. Kroner(1992). ARCH Modeling in finance. Journal of Econometrics, 52. Pp 5-59.

[17] Bollerslev, T., R.F. Engle and D.B. Nelson(1994). ARCH Models in R.F. Engle \& D.L. McFadden(eds) Handbook of Econometrics, vol. IV. Elsevier Science B.V.

[18] Bollerslev, T., R.F. Engle and J.M. Wooldridge(1988). A Capital Asset Pricing Model with Time Varying Covariances. Journal of Political Economy, 96, 1. Pp $116-130$. 
[19] Bonser-Neal, C., G.Brauer, R. Neal and S. Wheatley(1990). International Investment Restrictions and Closed-End Country Fund Prices. Journal of Finance, 45, 2. Pp 523-547.

[20] Branson, W. and D. Henderson(1985). The Specification and Influence of Asset Markets in Jones, R. and P. Kenen(eds) Handbook of International Economics, vol 2. Elsevier Science Publisher. Pp 749805.

[21] Breeden, D.T.(1979). An Intertemporal Asset Pricing Model with Stochastic Consumption and Investment Opportunities. Journal of Financial Economics, 7. Pp 265-296.

[22] Campbell, J.Y.(1987). Stock Returns and the Term Structure. Journal of Financial Economics, 18. Pp 373-399.

[23] Campbell,J.Y.and J. Ammer(1993). What Moves the Stock and Bond Markets? A Variance Decomposition for Long-Term Asset Returns. Journal of Finance, 48. Pp 3-37.

[24] Campbell, J.Y., A.W. Lo and A.C. MacKinlay(1997). The Econometrics of Financial Markets. Princeton University Press.

[25] Campbell, J.Y.and R.J. Shiller(1988). The Dividend-price ratio and Expectations of Future Dividends and Discount Factors. Review of Financial Studies, 1. Pp 195-228. 
[26] Clare, A.D., R.O'Brien, S.H. Thomas and M.R. Wickens(1998). Macroeconomic Shocks and the Domestic CAPM: Evidence from the UK Stock Market. International Journal of Finance and Economics, 3, Pp 111-126.

[27] Clare, A.D., P.N. Smith and S.H. Thomas(1996). Predicting UK Stock Returns and Robust Tests of Mean Variance Efficiency. University of York, working paper 96/22.

[28] Clare, A.D., and S.H. Thomas(1994). Macroeconomic Factors, the APT, and the UK Stockmarket. Journal of Business Finance and Accounting. Pp 309-330.

[29] Clare, A.D., S.H. Thomas and M.R. Wickens(1993). Is the Gilt-Equity Yield Ratio useful for predicting UK Stock Returns. Economic Journal, 104. Pp 303-315.

[30] Clarke, R.G. and H. de Silva(1998). State-Dependent Asset Allocation. Journal of Portfolio Management, 24. Pp 57-64.

[31] Constantinides, G.M. and A.G. Mallaris(1995). Portfolio Theory in R.A. Jarrow, V. Maksimovic and W.T. Ziemba(eds) Handbook of Finance. North Holland. 
[32] Cooper, I. and E. Kaplanis(1994). Home Bias in Equity Portfolios, Inflation Hedging, and International Capital Market Equilibrium. Review of Financial Studies, 7. Pp 45-60.

[33] Cumby, R.E.(1988). Is it Risk? Explaining Deviations from Uncovered Interest Parity. Journal of Monetary Economics, 22. Pp 279-300.

[34] Cumby, R., S. Figlewski and J. Hasbrouck(1994). International Asset Allocation with Time Varying risk: An Analysis and Implementation. Japan and the World Economy, 6, 1. Pp $1-25$.

[35] Day, T.E. and C.M. Lewis(1992). Stock Market and the Information Content of Stock Index Options. Journal of Econometrics, 52. Pp 247254.

[36] De Santis, G. and B. Gerard(1997). International Asset Pricing and Portfolio Diversification with Time-Varying Risk. Journal of Finance, 52. Pp 1881-1912.

[37] Diebold, F.X. and M. Nerlove(1989). The Dynamics of Exchange Rate Volatility: A Multivariate Latent Factor ARCH Model. Journal of Applied Econometrics, 4. Pp 1-21.

[38] Eaker, M.R. and D.M. Grant(1990). Currency Hedging Strategies for Internationally Diversified Equity Portfolios. Journal of Portfolio Management, 17. Pp $30-32$. 
[39] Eldor, R., D. Pines and A. Schwartz(1988). Home Asset Preference and Productivity Shocks. Journal of International Economics, 25. Pp $165-176$.

[40] Engel, C.(1984). Testing for the Absence of Expected Real Profits from Forward Market Speculation. Journal of International Economics, 25. Pp 299-308.

[41] Engel, C., J.A. Frankel, K.A. Froot and A.P. Rodrigues(1995). Tests of Conditional Mean-Variance Efficiency of the US Stock Market. Journal of Empirical Finance, 2. Pp 3 - 18.

[42] Engel, C. and A.P. Rodrigues(1989). Tests of International CAPM with Time-varying Covariances. Journal of Applied Econometrics, 4. Pp 119-138.

[43] Engle, R.F.(1982). Autoregressive Conditional Heteroskedasticity with estimates of the variance of UK Inflation. Econometrica, 50. Pp $987-1008$.

[44] Engle, R.F.(1987). Multivariate GARCH with Factor Structures Cointegration in Variance. Unpublished Manuscript, University of California, San Diego. 
[45] Engle, R.F., T.Ito and W.L. Lin(1990). Meteor Showers or Heat Waves? Heteroskedastic intra-daily Volatility in the Foreign Exchange Market. Econometrica, 58. Pp 525-542.

[46] Engle, R.F. and K.F. Kroner(1993). Multivariate Simultaneous Generalized ARCH. Unpublished Manuscript, University of California, San Diego.

[47] Engle, R.F. and C. Mustafa(1992). Implied ARCH Models from Option Prices. Journal of Econometrics, 52. Pp 289-311.

[48] Engle, R.F., V. Ng and M. Rothschild(1990). Asset Pricing with a Factor ARCH Covariance Structure: Empirical Estimates for Treasury Bills. Journal of Econometrics, 45. Pp 213-238.

[49] Eun, C. and B. Resnick(1988). Exchange Rate Uncertainty, Forward Contracts and International Portfolio Selection. Journal of Finance, 43. Pp 197-215.

[50] Eun, C. and B. Resnick(1994). International Diversification of Investment Portfolios: US and Japanese Perspectives. Management Science, 40. Pp 140 - 161.

[51] Fairlamb, D.(1989). The Elusive El Dorado of Spanish Pensions. Institutional Investor, April. Pp 177-184. 
[52] Fama, E.F.(1984). Stock Returns, Real Activity, Inflation and Money. American Economic Review, 71. Pp 545-564.

[53] Fama, E.F. and K.F. French(1989). Business Conditions and Expected Returns on Stocks and Bonds. Journal of Financial Economics, 25. Pp $23-49$.

[54] Fama, E.F. and K.R. French(1992). The Cross-Section of Expected Stock Returns. Journal of Finance, 47. Pp 427-465.

[55] Fama, E.F. and K.R. French(1995). Size and Book-to-Market Factors in Earnings and Returns. Journal of Finance, 50. Pp 131-155.

[56] Fama, E.F. and G.W. Schwert(1977). Asset Returns and Inflation. Journal of Financial Economics, 5. Pp 115-146.

[57] Fletcher, R.(1981). Practical Methods of Optimization: Vol.2 Constrained Optimization. John Wiley \& Sons.

[58] Frankel, J.A. and S.L. Schmukler(1996). Country Fund Discounts, Asymmetric Information and the Mexican Crisis of 1994: Did Local Residents turn Pessimistic before International Investors?. NBER working paper, No.5714. 
[59] French, K.R. and J.M. Poterba(1991). Investor Diversification and International Equity Markets. American Economic Review, 81,2. Pp $222-226$

[60] French, K.R., G.W. Schwert and R.F. Stambaugh(1987). Expected Stock Returns and Volatility. Journal of Financial Economics, 19. Pp 3-30.

[61] Gardner, G.W. and T. Wuillord(1995). Currency Risk in International Portfolios: How Satisfying is Optimal Hedging? Journal of Portfolio Management, 21. Pp 59 - 67.

[62] Geske, R. and R. Roll(1983). The Fiscal and Monetary Linkage between Stock Returns and Inflation. Journal of Finance, 38. Pp 1-33.

[63] Giovannini, A. and P. Jorion(1989). The Time Variation of Risk and Return in the Foreign Exchange and Stock Markets. Journal of Finance, 44. Pp $307-325$.

[64] Glen, J. and P. Jorion(1993). Currency Hedging for International Portfolios. Journal of Finance, 48, 5. Pp 1865 - 1886.

[65] Grauer, R.R. and N.H. Hakansson(1987). Gains from International Diversification: 1968-85 Returns on Portfolios of Stocks and Bonds. Journal of Finance, 42, 3. Pp 721 - 741. 
[66] Griffin, M.W.(1997). Why do Pension Funds and Insurance Portfolios hold so few International Assets? Journal of Portfolio Management, 23. $\mathrm{Pp} 45-50$.

[67] Groenewold, N., G. O'Rourke and S. Thomas(1997). Stock Returns and Inflation: a Macro Analysis. Applied Financial Economics, 7. Pp 127-136.

[68] Grubel, H.G.(1968). Internationally Diversified Portfolios: Welfare Gains and Capital Flows. American Economic Review, 58. Pp 1299 1314.

[69] Gultekin, N.B.(1983). Stock Market Returns and Inflation: evidence from other Countries. Journal of Finance, 38. Pp 49-65.

[70] Gultekin, M., B. Gultekin and A. Penati(1989). Capital Controls and International Capital Market Segmentation: The evidence from the Japanese and American Stock Markets. Journal of Finance, 44, 4. Pp 849-869.

[71] Halliday, L.(1989). The International Stock Exchange Directory. Institutional Investor, March. Pp 197-204.

[72] Heston, S.L. and K. Geert Rouwenhorst(1995). Industry and Country Effects in International Stock Returns. Journal of Portfolio Management, 21. Pp 53-58. 
[73] Hicks, J.R.(1939). Value and Capital. Oxford University Press, New York, NY.

[74] Hietala, P.(1989). Asset Pricing in Partially Segmented Markets: Evidence from the Finnish Market. Journal of Finance, 44, 3. Pp 697-718.

[75] Hodrick, R.J.(1992). Dividend Yields and Expected Stock Returns, Alternative procedures for Inference and Measurement. Review of Financial Studies, 5. Pp 357-386.

[76] Huang, C.F. and R.H. Litzenberger(1988). Foundations for Financial Economics. North Holland, Amsterdam.

[77] Ingersoll, J.E. Jr(1987). Theory of Financial Decision Making. Rowman and Littlefield, Totowa, NJ.

[78] Jankus, J.C.(1997). Relating Global Bond Yields to Macroeconomic Forecasts. Journal of Portfolio Management, 23. Pp 96-101.

[79] Jobson, J.D. and B. Korkie(1981). Putting Markowitz Theory to Work. Journal of Portfolio Management. Pp 70 - 74.

[80] Jorion, P.(1985). International Portfolio Diversification with Estimation Risk. Journal of Business, 58. Pp 259-278. 
[81] Kallberg, J.G. and W.T. Ziemba(1984). Mis-specifications in Portfolio Selection Problems in G.Bamberg and A. Spremann(eds) Risk and Capital. Springer-Verlag, New York, NY.

[82] Kang, J-K. and R.M. Stulz(1996). Why is there a home bias? An analysis of foreign Portfolio Equity ownership in Japan. Ohio State University, working paper no. 96-15.

[83] Keim, D.B. and R.F. Stambaugh(1986). Predicting Returns in the Stock and Bond Markets. Journal of Financial Economics, 17. Pp $357-390$.

[84] Kemp, L.(1987). The Wardley Guide to World Money and Securities Markets. Euromoney Publication Ltd., London.

[85] Keynes, J.L.(1936). The General Theory of Employment, Interest and Money. Harcourt Brace \& Co., New York, NY.

[86] Klemkosky, R.C. and R. Bharati(1995). Time-Varying Expected Returns and Asset Allocation. Journal of Portfolio Management, 21. Pp $80-87$.

[87] Kroll, Y., H. Levy and H.M. Markowitz(1984). Mean-Variance Versus Direct Utility Maximization. Journal of Finance, 39, 1. Pp 47 - 61. 
[88] Levy, H. and Z. Lerman(1988). The Benefits of International Diversification in Bonds. Financial Analysis Journal, 44. Pp 56-64.

[89] Levy H. and H.M. Markowitz(1979). Approximating Expected Utility by a function of Mean and Variance. American Economic Review, 69 . Pp 308 - 317.

[90] Levy, H. and M. Sarnat(1970). International Diversification of Investment Portfolios. American Economic Review, 60. Pp 668 - 675.

[91] Lintner, J.(1965). The Valuation of Risk Assets and the Selection of Risky Investments in Stock Portfolios and Capital Budgets. Review of Economics and Statistics, 47. Pp 13-37.

[92] Markowitz, H.M.(1952). Portfolio Selection. Journal of Finance, 46. Pp 469 - 477.

[93] Markowitz, H.M.(1959). Portfolio Selection: Efficient Diversification of Investments. Wiley, New York, NY.

[94] McCurdy, T.H. and I. Morgan(1991). Evidence of Risk Premia in foreign currency futures markets. Review of Financial Studies.

[95] Merton, R.C.(1987). A Simple Model of Capital Market Equilibrium with Incomplete Information. Journal of Finance, 42. Pp 483-510. 
[96] Nelson, C.R.(1976). Inflation and Rates of Return on Common Stock. Journal of Finance, 31. Pp 471-483.

[97] Ng, L.(1991). Tests of the CAPM with time varying covariances: A Multivariate GARCH approach. Journal of Finance, 46. Pp1507-1521.

[98] Ng, V., R.F. Engle and M. Rothschild(1992). A Multi-Dynamic Factor Model for Stock Returns. Journal of Econometrics, 52. Pp 245-265.

[99] Patelis, A.D.(1997). Stock Return Predictability and the Role of Monetary Policy. Journal of Finance, 52. Pp 1951-1972.

[100] Pesaran, M.H. and A. Timmermann(1995). Predictability of Stock Returns: Robustness and Economic Significance. Journal of Finance, 50. $\mathrm{Pp}$ 1201-1228.

[101] Poterba, J.M. and L. Summers(1988). Mean Reversion in Stock Prices: Evidence and Implications. Journal of Financial Economics, 22. Pp 27 -59 .

[102] Pulley, L.B.(1981). A General Mean-Variance Approximation to Expected Utility for Short Holding Periods. Journal of Financial and Quantitative Analysis, 16, 3. Pp 361 - 373.

[103] Roll, R.(1977). A Critique of the Asset Pricing Theory's Tests: Part 1. Journal of Financial Economics, 4. Pp 129 - 176. 
[104] Roll, R. and S.A. Ross(1986). Economic Forces and the Stock Market. Journal of Business, 59. Pp 383-406.

[105] Rozeff, M.(1984). Dividend Yields and Equity Risk Premiums. Journal of Portfolio Management, 11. Pp 68-75.

[106] Schwert, G.W.(1989). Why Does Stock Market Volatility Change Over Time? Journal of Finance, 44. Pp 1115-1153.

[107] Schwert, G.W. and P.J. Seguin(1990). Heteroskedasticity in Stock Returns. Journal of Finance, 45. Pp 1129-1155.

[108] Sercu, P.(1980). A Generalization of the International Asset Pricing Model. Revue de l'Association Francaise de Finance, 1. Pp 91-135.

[109] Sharpe, W.F.(1964). Capital Asset Prices: A Theory of Market Equilibrium under Conditions of Risk. Journal of Finance, 19. Pp 425-442.

[110] Shiller, R.J., F. Kon-ya and Y. Tsutsui(1990). Speculative Behaviour in the Stock Markets: Evidence from the US and Japan. Mimeo., Yale University.

[111] Singleton, K.(1991). Specification and Estimation of Intertemporal Asset Pricing Models in Friedman, B. and F. Hahn(eds) Handbook of Monetary Economics. North Holland, Amsterdam. 
[112] Solnik, B.(1983). The Relation between Stock prices and Inflationary expectations: the International evidence. Journal of Finance, 39, 4. Pp 35-48.

[113] Stockman, A. and H. Dellas(1989). International Portfolio Nondiversification and Exchange Rate Variability. Journal of International Economics, 26. Pp 271-289.

[114] Tesar, L.L. and I.M. Werner(1995). Home Bias and High Turnover. Journal of International Money and Finance, 14, 4. Pp 467-492.

[115] Thomas, S.H. and M.R. Wickens(1993). An International CAPM for Bonds and Equities. The Journal of International Money and Finance, 12. Pp $390-412$.

[116] Tobin, J.(1958). Liquidity Preference as behaviour toward Risk. Review of Economic Studies, 25. Pp $65-86$.

[117] Tversky, A. and C. Heath(1991). Preferences and Beliefs: Ambiguity and Competence in Choice under Uncertainty. Journal of Risk and Uncertainty, 4. Pp 5-28.

[118] Uppal, R.(1992). The Economic Determinants of the Home Country Bias in Investors' Portfolios: A Survey. Journal of International Financial Management and Accounting, 4. Pp 171-189. 
[119] Uppal, R.(1993). A General Equilibrium Model of International Portfolio Choice. Journal of Finance, 48,2. Pp 529-553. 